\title{
Polarizing the Nazarov Cyclization: The Impact of Dienone Substitution Pattern on Reactivity and Selectivity
}

\author{
Wei He, Ildiko R. Herrick, Tulay A. Atesin, Patrick A. Caruana, \\ Colleen A. Kellenberger, Alison J. Frontier* \\ Department of Chemistry, University of Rochester, Rochester, NY 14627
}

\section{Supporting Information Part I}

\section{TABLE OF CONTENTS}

General Methods..........................................

Preparation of Compounds in Table $1 \ldots \ldots \ldots \ldots \ldots \ldots \ldots \ldots \ldots \ldots . .2$

Preparation of Compounds in Table $2 \ldots \ldots \ldots \ldots \ldots \ldots \ldots \ldots \ldots . . .6$

Preparation of Compounds in Table $3 \ldots \ldots \ldots \ldots \ldots \ldots \ldots \ldots \ldots . . . . . . . . . .11$

Preparation of Compounds in Table 4...........................16

Preparation of Compounds in Table $6 \ldots \ldots \ldots \ldots \ldots \ldots \ldots \ldots \ldots . .21$

Preparation of Alkynes S7-S11 .............................21

Preparation of Compounds in Table $7 \ldots \ldots \ldots \ldots \ldots \ldots \ldots \ldots \ldots \ldots . .25$

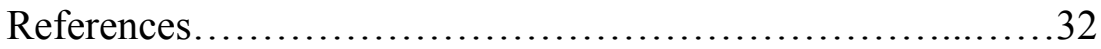


General Methods: All reactions were carried out under an atmosphere of argon in ovendried glassware with magnetic stirring. $\mathrm{Cu}(\mathrm{OTf})_{2}$ was stored in a glove box before use and used as purchased from Aldrich. $\mathrm{Cu}\left(\mathrm{ClO}_{4}\right)_{2}$ was dried in a vacuum oven at $40^{\circ} \mathrm{C}$ for $24 \mathrm{~h}$, then ground and stored in a glovebox prior to use. Dichloroethane was purchased from Fisher and dispensed using the Glass Contour solvent purification system. Column chromatography was performed on EM Science silica gel 60 (230-400 mesh).

Visualization was done with UV light and potassium permanganate, $p$-anisaldehyde or ceric ammonium molybdate solution followed by heating. Infrared spectra were recorded on a ATI Mattson Genesis FT-IR spectrometer. ${ }^{1} \mathrm{H}$ NMR and ${ }^{13} \mathrm{C}$ NMR spectra were collected on a Bruker AMX $400 \mathrm{MHz}$ or on an Avance $400 \mathrm{MHz}$ spectrometer at ambient temperature. Chemical shift $\delta$ was reported in units of parts per million downfield from tetramethylsilane. HRMS was done on a ThermoFinnigan MAT 95XL with electrospray with $50 \% \mathrm{MeOH}$ at the Chemistry Instrumentation Center of the University of Buffalo.

Spectroscopic Data of Substrates: Structural assignment, including the identification of $\mathrm{E} / \mathrm{Z}$ isomers and cis/trans isomers, was determined by either NMR spectroscopy on a Bruker $500 \mathrm{MHz}$ spectrometer or an Avance $400 \mathrm{MHz}$ spectrometer (including nOe experiments, selective ${ }^{1} \mathrm{H}$-decoupled ${ }^{13} \mathrm{C}$ experiments, ${ }^{1}$ or both, as applicable) or X-ray crystal structure.

\section{Preparation of Compounds in Table 1:}

General Procedure I: Knoevenagel Condensation. ${ }^{2}$ To $50 \mathrm{~mL}$ round bottom flask containing a solution of $\beta$-ketoester $\mathbf{S 1}$ (460 mg, $2.5 \mathrm{mmol}, 1$ equiv.) in $\mathrm{PhH}$ ( $12.5 \mathrm{~mL}$, $0.2 \mathrm{M})$ at r.t. was added acetic acid $(90 \mathrm{mg}, 1.5 \mathrm{mmol}, 0.6$ equiv. $)$, piperidine ( $21 \mathrm{mg}$, $0.25 \mathrm{mmol}, 0.1$ equiv.) and the proper aldehyde ( $2.25 \mathrm{mmol}, 0.9$ equiv.) sequentially. The flask was fitted with a Dean-Stark trap and a condenser. After flushed with argon, this flask was lowered into an oil-bath preheated to $100{ }^{\circ} \mathrm{C}$. The reaction was refluxed for 1 hour upon when TLC found complete consumption of the ketoester S1. The reaction was allowed to cool down to r.t. and diluted with $5 \mathrm{~mL} \mathrm{NaHCO}_{3}$ (aq.). The organic phase was separated and the aqueous phase was extracted with ether ( $5 \mathrm{~mL} \mathrm{X} 3$ times). The combined organic phase was dried over $\mathrm{MgSO}_{4}$, concentrated under reduced pressure. The residue was purified by flash chromatography $(50 \% \mathrm{v} / \mathrm{v}$ EtOAc/hexanes) to offer the desired alkylidene $\beta$-ketoester 6 as a pale yellow oil ( $450 \mathrm{mg}, 1.25 \mathrm{mmol}, 50 \%$ ).

Compounds 2-alkoxy-4-carboalkoxy-1,4-pentadien-3-ones were prepared through Knoevanegel condensation between $\beta$-ketoester S1 and a proper aldehyde following General Procedure I.

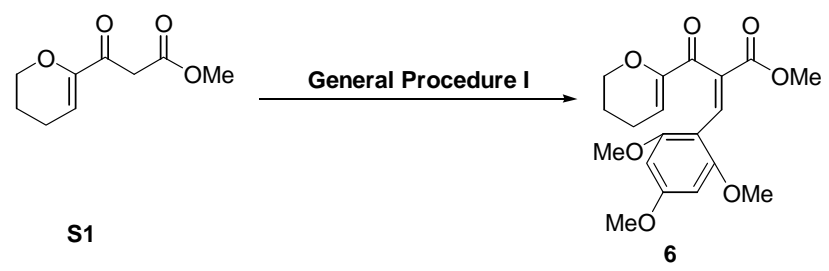


${ }^{1}$ H NMR (400 MHz, CDCl $) \delta 7.90(\mathrm{~s}, 1 \mathrm{H}), 5.98(\mathrm{~s}, 2 \mathrm{H}), 5.79(\mathrm{~s}, 1 \mathrm{H}), 3.98(\mathrm{t}, J=$ $4.6 \mathrm{~Hz}, 2 \mathrm{H}), 3.74(\mathrm{~s}, 3 \mathrm{H}), 3.71(\mathrm{~s}, 3 \mathrm{H}), 3.66(\mathrm{~s}, 6 \mathrm{H}), 2.06(\mathrm{~d}, J=4.5 \mathrm{~Hz}, 2 \mathrm{H}), 1.75(\mathrm{t}, J=$ $4.4 \mathrm{~Hz}, 2 \mathrm{H}) ;{ }^{13} \mathbf{C}$ NMR (CDCl 3 , 100 MHz) $\delta 188.6,166.7,163.4,159.3,151.1,135.3$, 127.5, 111.1, 104.8, 90.0, 66.1, 55.2, 54.9, 51.8, 21.6, 20.6; IR (NaCl plate, $\mathbf{~ c m}^{-1}$ ): 1712, $1603,1460,1336,1255,1206,1156,1127,1056$; Elemental analysis calculated for $\mathrm{C}_{19} \mathrm{H}_{22} \mathrm{O}_{7}$ : C 62.97\%; H 6.12\%. Found : C 62.79\%; H 6.20\%.

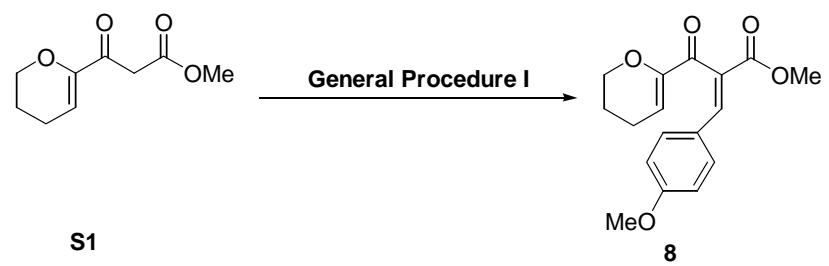

${ }^{1}$ H NMR (400 MHz, $\left.\mathbf{C D C l}_{3}\right) \delta 7.59(\mathrm{~s}, 1 \mathrm{H}), 7.16(\mathrm{~d}, J=8.8 \mathrm{~Hz}, 2 \mathrm{H}), 6.67(\mathrm{~d}, J=$ $8.8 \mathrm{~Hz}, 2 \mathrm{H}), 5.87(\mathrm{t}, J=4.4 \mathrm{~Hz}, 1 \mathrm{H}), 3.93(\mathrm{t}, J=5.6 \mathrm{~Hz}, 2 \mathrm{H}), 3.62(\mathrm{~s}, 6 \mathrm{H}), 1.98(\mathrm{~m}, 2 \mathrm{H})$, $1.65(\mathrm{t}, J=6 \mathrm{~Hz}, 2 \mathrm{H}) ;{ }^{13} \mathbf{C}$ NMR $\left(\mathbf{C D C l}_{3}, \mathbf{1 0 0} \mathbf{M H z}\right) \delta 190.7,165.3,161.2,150.9,142.5$, 132.0, 127.1, 125.2, 116.2, 114.0, 66.3, 55.1, 52.0, 21.0, 20.8; IR (NaCl plate, $\mathbf{~ c m}^{-1}$ ): $1714,1601,1512,1259,1204,1175,734$; HRMS calculated for $\mathrm{C}_{17} \mathrm{H}_{18} \mathrm{O}_{5}(\mathrm{M}+\mathrm{H})^{+}$ 303.1224, Found : 303.1227.

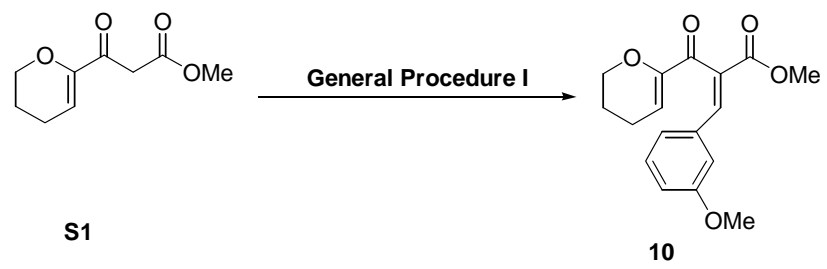

${ }^{1} \mathbf{H}$ NMR $\left(400 \mathrm{MHz}, \mathbf{C D C l}_{3}\right) \delta 7.58(\mathrm{~s}, 1 \mathrm{H}), 7.03(\mathrm{t}, \mathrm{J}=3.6 \mathrm{~Hz}, 1 \mathrm{H}), 6.75(\mathrm{~d}, J=9.6$ $\mathrm{Hz}, 1 \mathrm{H}), 6.69(\mathrm{~s}, 2 \mathrm{H}), 5.81(\mathrm{t}, J=4.4 \mathrm{~Hz}, 1 \mathrm{H}), 3.86(\mathrm{t}, J=4.6 \mathrm{~Hz}, 2 \mathrm{H}), 3.58(\mathrm{~s}, 3 \mathrm{H}), 3.53$ $(\mathrm{s}, 3 \mathrm{H}), 1.94(\mathrm{~d}, J=6 \mathrm{~Hz}, 2 \mathrm{H}), 1.57(\mathrm{t}, J=6.0 \mathrm{~Hz}, 2 \mathrm{H}) ;{ }^{13} \mathbf{C} \mathbf{~ N M R}\left(\mathbf{C D C l}_{3}, \mathbf{1 0 0} \mathbf{M H z}\right) \delta$ 189.9, 164.8, 159.3, 150.9, 142.6, 133.9, 130.3, 129.5, 122.4, 116.4, 115.8, 114.3, 66.2, 54.7, 52.1, 21.0, 20.7; IR (NaCl plate, .m $^{-1}$ ): 1715, 1671, 1624, 1236, 1051; HRMS calculated for $\mathrm{C}_{17} \mathrm{H}_{18} \mathrm{O}_{5}(\mathrm{M}+\mathrm{H})^{+} 303.1233$ Found : 303.1227 .

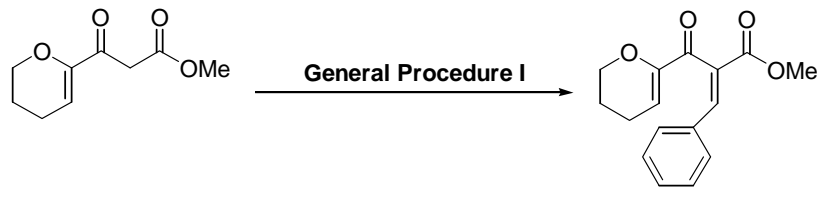

S1

${ }^{1}$ H NMR (400 MHz, $\left.\mathbf{C D C l}_{3}\right) \delta 7.58(\mathrm{~s}, 1 \mathrm{H}), 7.13(\mathrm{~m}, 2 \mathrm{12}), 7.04(\mathrm{~m}, 3 \mathrm{H}), 5.77(\mathrm{t}, J=$ $4.1 \mathrm{~Hz}, 1 \mathrm{H}), 3.79(\mathrm{t}, J=4.4 \mathrm{~Hz}, 2 \mathrm{H}), 3.53(\mathrm{~s}, 3 \mathrm{H}), 1.86(\mathrm{~m}, 2 \mathrm{H}), 1.52(\mathrm{~m}, 2 \mathrm{H}) ;{ }^{13} \mathbf{C}$ NMR $\left(\mathbf{C D C l}_{3}, \mathbf{1 0 0} \mathbf{M H z}\right) \delta 189.9,164.8,150.8,142.6,132.6,131.1,129.8,128.4,115.9,66.1$, 52.1, 52.1, 20.9, 20.6; IR (NaCl plate, $\mathbf{~ c m}^{-1}$ ): 1714, 1257, 1201, 1051; Elemental analysis calculated for $\mathrm{C}_{16} \mathrm{H}_{16} \mathrm{O}_{4}$ : $\mathrm{C} 70.57 \%$; $\mathrm{H}$ 5.92\%. Found : $\mathrm{C} 70.80 \% ; \mathrm{H} 6.02 \%$. 


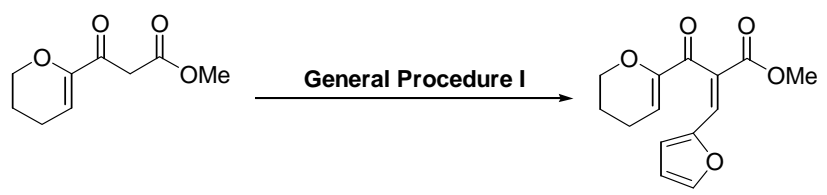

S1

14

${ }^{1}$ H NMR (400 MHz, $\left.\mathbf{C D C l}_{3}\right) \delta 7.34(\mathrm{~s}, 1 \mathrm{H}), 7.28(\mathrm{~d}, J=1.2 \mathrm{~Hz}, 1 \mathrm{H}), 6.51(\mathrm{~d}, J=$ $3.6 \mathrm{~Hz}, 1 \mathrm{H}), 6.26(\mathrm{q}, J=1.6 \mathrm{~Hz}, 1 \mathrm{H}), 5.77(\mathrm{t}, J=3.6 \mathrm{~Hz}, 1 \mathrm{H}), 3.91(\mathrm{t}, J=4.8 \mathrm{~Hz}, 2 \mathrm{H})$, $3.56(\mathrm{~s}, 3 \mathrm{H}), 1.98(\mathrm{~m}, 2 \mathrm{H}), 1.63(\mathrm{t}, J=5.2 \mathrm{~Hz}, 2 \mathrm{H}) ;{ }^{13} \mathbf{C} \mathbf{~ N M R}\left(\mathbf{C D C l}_{3}, \mathbf{1 0 0} \mathbf{~ M H z}\right) \delta$ 189.0, 164.9, 151.1, 148.9, 146.0, 128.2, 125.8, 117.8, 115.5, 112.4, 66.2, 52.1, 22.1, 20.7; IR (NaCl plate, $\mathbf{~ c m}^{-1}$ ): 1710, 1671, 1626, 1253, 1212; Elemental analysis calculated for $\mathrm{C}_{14} \mathrm{H}_{14} \mathrm{O}_{5}$ : C 64.12\%; H 5.38\%. Found : C 63.83\%; H 5.42\%.

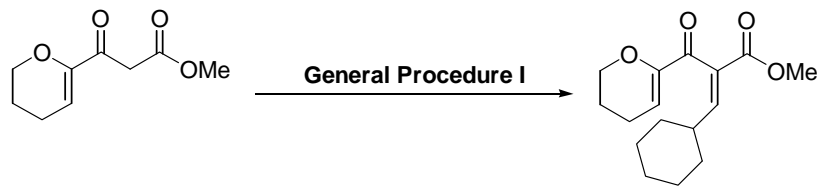

S1

16

${ }^{1}$ H NMR (400 MHz, $\left.\mathbf{C D C l}_{3}\right) \delta 6.69(\mathrm{~d}, J=10.8 \mathrm{~Hz}, 1 \mathrm{H}), 5.85(\mathrm{t}, J=4.4 \mathrm{~Hz}, 1 \mathrm{H})$, $3.99(\mathrm{t}, J=5.2 \mathrm{~Hz}, 2 \mathrm{H}), 3.60(\mathrm{~s}, 3 \mathrm{H}), 2.08(\mathrm{~m}, 2 \mathrm{H}), 2.03(\mathrm{~m}, 1 \mathrm{H}), 1.76(\mathrm{~m}, 2 \mathrm{H}), 1.54(\mathrm{~m}$, 5H), $1.02(\mathrm{~m}, 5 \mathrm{H}) ;{ }^{\mathbf{1 3}} \mathbf{C}$ NMR (CDCl 3 , $\left.\mathbf{1 0 0} \mathbf{M H z}\right) \delta$ 189.1, 164.9, 152.7, 151.5, 130.4, 115.4, 66.2, 51.8, 38.4 , 31.3, 25.3, 24.8, 21.1, 20.8; IR (NaCl plate, $\left.\mathbf{~ c m}^{-1}\right)$ ) 2925, 2853, $1738,1713,1645,1444,1300,1266,1216,1162,1123,1071,1034$; Elemental analysis calculated for $\mathrm{C}_{16} \mathrm{H}_{22} \mathrm{O}_{4}$ : C 69.04\%; H 7.97\%. Found : C 69.17\%; H 7.79\%.
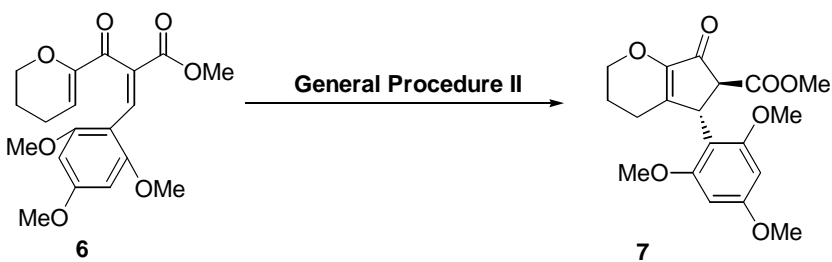

General Procedure II: Nazarov Cyclization Using Cu(OTf $)_{2}$ : To $25 \mathrm{~mL}$ round bottom flask containing a suspension of $\mathrm{Cu}(\mathrm{OTf})_{2}(2.0 \mathrm{mg}, 0.006 \mathrm{mmol}, 0.02$ equiv. ) in $5 \mathrm{~mL}$ of dichloromethane under argon was added a solution of the substrate 6 (100 mg, 0.27 mmol, 1.0 equiv.) in dichloromethane $(5.2 \mathrm{~mL}, 0.2 \mathrm{M})$ at r.t.. The reaction was monitered by TLC. After the s.m. was completely consumed, the reaction was diluted with $5 \mathrm{~mL}$ $\mathrm{NaHCO}_{3}$ (aq.). The organic phase was separated and the aqueous phase was extracted with ether (5 $\mathrm{mL}$ X 3 times). The combined organic phase was dried over $\mathrm{MgSO}_{4}$, concentrated under reduced pressure. The residue was purified by flash chromatography $(50 \% \mathrm{v} / \mathrm{v}$ EtOAc/hexanes) to offer the desired Nazarov product 7.

${ }^{1}$ H NMR (400 MHz, $\left.\mathbf{C D C l}_{3}\right) \delta 6.80\left(\mathrm{dd}, J_{1}=6.4 \mathrm{~Hz}, J_{2}=3.2 \mathrm{~Hz}, 1 \mathrm{H}\right), 6.14(\mathrm{~s}, 2 \mathrm{H})$, $4.30(\mathrm{~d}, J=12.1 \mathrm{~Hz}, 1 \mathrm{H}), 3.80(\mathrm{t}, J=12.1 \mathrm{~Hz}, 1 \mathrm{H}), 3.77(\mathrm{~s}, 3 \mathrm{H}), 3.74(\mathrm{~s}, 6 \mathrm{H}), 3.65(\mathrm{~s}$, $3 \mathrm{H}), 2.93(\mathrm{~m}, 1 \mathrm{H}), 2.04(\mathrm{~m}, 2 \mathrm{H}), 1.80(\mathrm{~m}, 2 \mathrm{H}), 1.25(\mathrm{~m}, 1 \mathrm{H}), 1.13(\mathrm{~m}, 1 \mathrm{H}) ;{ }^{13} \mathbf{C} \mathbf{N M R}$ $\left(\mathbf{C D C l}_{3}, \mathbf{1 0 0}\right.$ MHz) $\delta 199.4,170.3,159.9,159.7,140.6,134.4,107.5,91.1,91.0,57.6$, 55.2, 55.0, 52.0, 40.4 , 39.6, 27.1, 25.6, 21.5; IR (NaCl plate, $\mathbf{~ c m}^{-1}$ ): 1742, 1711, 1606, $1459,1202,1151,1115$; Elemental analysis calculated for $\mathrm{C}_{20} \mathrm{H}_{24} \mathrm{O}_{6}: \mathrm{C} 66.65 \% ; \mathrm{H}$, 
$6.71 \%$. Found : C $66.80 \%$; H $6.75 \%$. X-ray crystal structure is available for this compound.
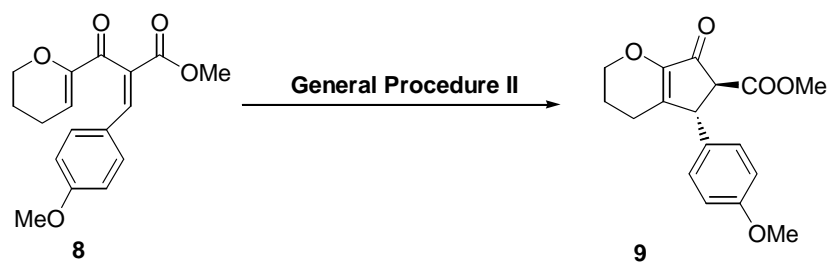

${ }^{1}$ H NMR (400 MHz, $\left.\mathbf{C D C l}_{3}\right) \delta 7.01(\mathrm{~d}, J=8.8 \mathrm{~Hz}, 2 \mathrm{H}), 6.83(\mathrm{~d}, J=8.8 \mathrm{~Hz}, 2 \mathrm{H})$, $4.13(\mathrm{~m}, 3 \mathrm{H}), 3.73(\mathrm{~s}, 3 \mathrm{H}), 3.71(\mathrm{~s}, 3 \mathrm{H}), 3.24(\mathrm{~d}, J=2.4 \mathrm{~Hz}, 1 \mathrm{H}), 2.10(\mathrm{~m}, 2 \mathrm{H}), 1.90(\mathrm{~m}$, $2 \mathrm{H}) ;{ }^{13} \mathbf{C}$ NMR (CDCl $\left.{ }_{3}, \mathbf{1 0 0} \mathbf{~ M H z}\right) \delta 193.0,168.7,158.9,149.5,147.8,131.5,128.2$, 114.4, 66.9, 59.3, 55.2, 52.6, 46.8, 22.0, 22.1; IR (NaCl plate, $\mathbf{~ c m}^{-1}$ ): 1738, 1713, 1648, 1512, 1250, 1162, 1122; HRMS calculated for $\mathrm{C}_{19} \mathrm{H}_{22} \mathrm{O}_{7}(\mathrm{M}+\mathrm{H})^{+}$303.1216, Found : 303.1227 .

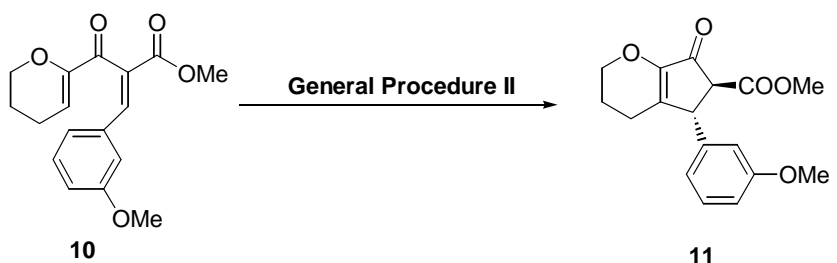

${ }^{1} \mathbf{H}$ NMR (400 MHz, $\left.\mathbf{C D C l}_{3}\right) \delta 7.23(\mathrm{t}, J=8 \mathrm{~Hz}, 1 \mathrm{H}), 6.78\left(\mathrm{dd}, J_{1}=8 \mathrm{~Hz}, J_{2}=6\right.$ $\mathrm{Hz}, 1 \mathrm{H}), 6.69(\mathrm{~d}, J=7.6 \mathrm{~Hz}, 1 \mathrm{H}), 6.64(\mathrm{~d}, J=1.6 \mathrm{~Hz}, 1 \mathrm{H}), 4.16$ (br. s, 1H), 4.08 (m, 2H), 3.75 (s, 3H), 3.73 (s, 3H), 3.29 (br. s, 1H), 2.10 (m, 2H), 1.88 (m, 2H); ${ }^{13}$ C NMR $\left(\mathbf{C D C l}_{3}, 100 \mathrm{MHz}\right) \delta 192.8,168.6,160.0,149.7,147.3,141.3,130.1,119.4,113.2$, 112.6, 66.9, 58.9, 58.9, 55.1, 47.5, 22.1, 21.1; IR (NaCl plate, $\mathbf{~ c m}^{-1}$ ): 1736, 1714, 439.3; Elemental analysis calculated for $\mathrm{C}_{17} \mathrm{H}_{18} \mathrm{O}_{5}$ : $\mathrm{C} 67.54 \% ; \mathrm{H} 6.00 \%$. Found : $\mathrm{C} 67.62 \% ; \mathrm{H}$ $5.94 \%$.

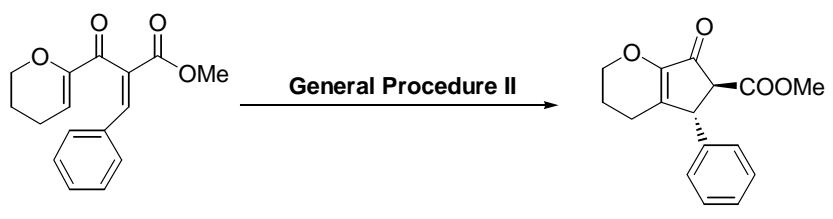

12

13

${ }^{1} \mathbf{H}$ NMR (400 MHz, $\left.\mathbf{C D C l}_{3}\right) \delta 7.33(\mathrm{t}, J=7.2 \mathrm{~Hz}, 2 \mathrm{H}), 7.28(\mathrm{~d}, J=7.2 \mathrm{~Hz}, 1 \mathrm{H})$, $7.13(\mathrm{~d}, J=7.2 \mathrm{~Hz}, 2 \mathrm{H}), 4.20$ (br. s, $1 \mathrm{H}), 4.15$ (m, 2H), 3.75 (s, 3H), 3.32 (br. s, $1 \mathrm{H}$ ), $2.13(\mathrm{~m}, 1 \mathrm{H}), 2.08(\mathrm{~m}, 1 \mathrm{H}), 1.93(\mathrm{~m}, 2 \mathrm{H}) ;{ }^{13} \mathbf{C} \mathbf{~ N M R}\left(\mathbf{C D C l}_{3}, \mathbf{1 0 0} \mathbf{~ M H z}\right) \delta 192.8,168.6$, 149.7, 147.4, 139.7, 128.8, 127.6, 127.2, 66.9, 59.1, 52.8, 47.5, 22.1, 21.1; IR (NaCl plate, $\mathbf{c m}^{-1}$ ): $1739,1714,1648,1263,1161,1123$; Elemental analysis calculated for $\mathrm{C}_{16} \mathrm{H}_{16} \mathrm{O}_{4}$ : C 70.57\%; H 5.92\%. Found : C 70.70\%; H 5.78\%.

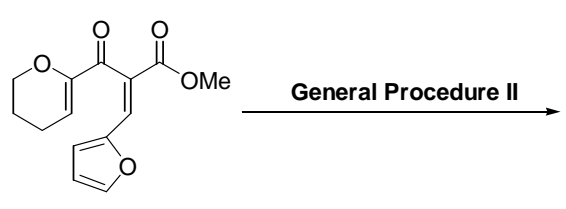

14

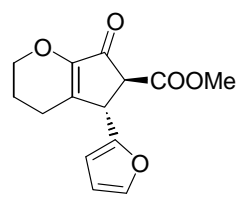

15 
${ }^{1} \mathbf{H}$ NMR (400 MHz, $\left.\mathbf{C D C l}_{3}\right) \delta 7.33(\mathrm{~d}, J=1.2 \mathrm{~Hz}, 1 \mathrm{H}), 6.31(\mathrm{t}, J=1.2 \mathrm{~Hz}, 1 \mathrm{H})$, $6.17(\mathrm{~d}, J=3.2 \mathrm{~Hz}, 1 \mathrm{H}), 4.35(\mathrm{~d}, J=2 \mathrm{~Hz}, 1 \mathrm{H}), 4.13(\mathrm{~m}, 2 \mathrm{H}), 3.76(\mathrm{~s}, 3 \mathrm{H}), 3.52(\mathrm{~d}, J=2$ $\mathrm{Hz}, 1 \mathrm{H}), 2.27(\mathrm{~m}, 1 \mathrm{H}), 2.19(\mathrm{~m}, 1 \mathrm{H}), 1.94(\mathrm{~m}, 2 \mathrm{H}) ;{ }^{13} \mathbf{C} \mathbf{N M R}\left(\mathbf{C D C l}_{3}, \mathbf{1 0 0} \mathbf{M H z}\right) \delta$ 192.0, 168.4, 151.7, 149.4, 144.7, 142.4, 110.3, 107.2, 66.9, 55.5, 52.8, 40.8, 22.2 21.1; IR (NaCl plate, $\mathbf{c m}^{-1}$ ): $1715,1649,524$; Elemental analysis calculated for $\mathrm{C}_{14} \mathrm{H}_{14} \mathrm{O}_{5}$ : $\mathrm{C}$ $64.12 \%$; H 5.38\%. Found : C 64.00\%; H 5.51\%.

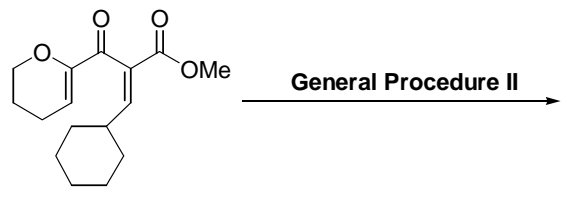

16

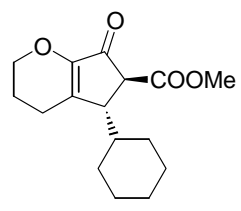

17

${ }^{1}$ H NMR (400 MHz, CDCl $) \delta 4.16(\mathrm{~m}, 1 \mathrm{H}), 4.03(\mathrm{~m}, 1 \mathrm{H}), 3.72(\mathrm{~s}, 3 \mathrm{H}), 3.17(\mathrm{~d}, J=$ $2.4 \mathrm{~Hz}, 1 \mathrm{H}), 3.03(\mathrm{~d}, J=2.4 \mathrm{~Hz}, 1 \mathrm{H}), 2.33(\mathrm{~m}, 2 \mathrm{H}), 1.93(\mathrm{~m}, 2 \mathrm{H}), 1.69-1.74(\mathrm{~m}, 4 \mathrm{H})$, 1.07-1.24 (m, 7H); ${ }^{13} \mathbf{C}$ NMR (CDCI, 100 MHz) $\delta 194.1,170.0,149.1,147.8,66.8$, 52.6, 51.5, 47.4, 38.2, 31.0, 26.6, 26.3, 26.1, 26.0, 22.7, 21.2; IR (NaCl plate, $\mathbf{~ c m}^{-1}$ ): $2359,2337,1711,1033,824,614$; Elemental analysis calculated for $\mathrm{C}_{16} \mathrm{H}_{22} \mathrm{O}_{4}$ : C $69.04 \%$; H 7.97\%. Found : C 68.84\%; H 7.93\%.

\section{Preparation of Compounds in Table 2}

The following compounds were prepared by Knoevenagel condensation following General Procedure I:

Knoevenagel condensation gave a mixture of isomers with a ratio of 95/5 (18E/18Z).<smiles>COc1cc(OC)c(/C=C(\C(C)=O)C(=O)C2=CCCCC2)c(OC)c1</smiles>

E-2-(Cyclohex-1-enecarbonyl)-3-(2,4,6-trimethoxy-phenyl)-acrylic acid methyl ester (18E). Purified by gravity chromatography (silica gel, ethyl acetate: hexanes $=1: 2) .{ }^{1} \mathrm{H}$ NMR (400 MHz, $\left.\mathrm{CDCl}_{3}\right): \delta 1.52(\mathrm{~m}, 4 \mathrm{H}), 2.04(\mathrm{~m}, 2 \mathrm{H}), 2.25(\mathrm{~m}, 2 \mathrm{H}), 3.71(\mathrm{~s}, 6 \mathrm{H})$, $3.76(\mathrm{~s}, 3 \mathrm{H}), 3.78(\mathrm{~s}, 3 \mathrm{H}), 6.06(\mathrm{~s}, 2 \mathrm{H}), 6.62(\mathrm{~s}, 1 \mathrm{H}), 7.98(\mathrm{~s}, 1 \mathrm{H}) ;{ }^{13} \mathrm{C}$ NMR $(100 \mathrm{MHz}$, $\left.\mathrm{CDCl}_{3}\right): \delta 21.6,21.8,22.8,25.8,51.9,54.9,55.2,89.9,105.3,128.4,134.9,138.2,141.5$, 159.3, 163.1, 167.3, 194.8; IR ( $\mathrm{NaCl}$ plate, $\left.\mathrm{cm}^{-1}\right)$ : 1710, 1603, 1251, 1206, 1157, 1128; Elemental analysis calculated for $\mathrm{C}_{20} \mathrm{H}_{24} \mathrm{O}_{6}$ : C 66.65\%; H, 6.71\%. Found : $\mathrm{C} 66.48 \% ; \mathrm{H}$ $6.56 \%$.

$\boldsymbol{E}^{3} \mathrm{~J}_{\mathrm{C}-\mathrm{H}}=9.2 \mathrm{~Hz}$ for $\mathrm{C}_{\mathrm{ketone}}-\mathrm{H},{ }^{3} \mathrm{~J}_{\mathrm{C}-\mathrm{H}}=8.4 \mathrm{~Hz}$ for $\mathrm{C}_{\text {ester }}-\mathrm{H}$. 


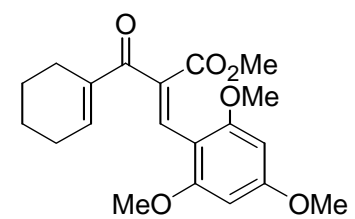

Z-2-(Cyclohex-1-enecarbonyl)-3-(2,4,6-trimethoxy-phenyl)-acrylic acid methyl ester (18Z). Purified by gravity chromatography (silica gel, ethyl acetate: hexanes $=1: 2$ ). ${ }^{1} \mathrm{H}$ NMR (400 MHz, $\left.\mathrm{CDCl}_{3}\right): \delta 1.60(\mathrm{~m}, 4 \mathrm{H}), 2.16(\mathrm{~m}, 2 \mathrm{H}), 2.27(\mathrm{~m}, 2 \mathrm{H}), 3.60(\mathrm{~s}, 3 \mathrm{H})$, $3.69(\mathrm{~s}, 6 \mathrm{H}), 3.75(\mathrm{~s}, 3 \mathrm{H}), 6.02(\mathrm{~s}, 2 \mathrm{H}), 6.69(\mathrm{~s}, 1 \mathrm{H}), 7.16(\mathrm{~s}, 1 \mathrm{H}) ;{ }^{13} \mathrm{C}$ NMR $(100 \mathrm{MHz}$, $\left.\mathrm{CDCl}_{3}\right): \delta 21.4,21.8,23.6,25.8,51.3,54.9,55.3,90.1,105.2,132.5,134.4,139.0,141.9$, 159.1, 163.0, 167.8, 195.3; IR (NaCl plate, $\left.\mathrm{cm}^{-1}\right): 2921,2210,1705,1600,1211,1144$, 1128, 830; Elemental analysis calculated for $\mathrm{C}_{20} \mathrm{H}_{24} \mathrm{O}_{6}$ : C 66.65\%; H, 6.71\%. Found : C $66.56 \%$; H $6.66 \%$.

$Z^{3} \mathrm{~J}_{\mathrm{C}-\mathrm{H}}=6.5 \mathrm{~Hz}$ for $\mathrm{C}_{\text {ketone }}-\mathrm{H},{ }^{3} \mathrm{~J}_{\mathrm{C}-\mathrm{H}}=12.8 \mathrm{~Hz}$ for $\mathrm{C}_{\text {ester }}-\mathrm{H}$.

Knoevenagel condensation gave a mixture of isomers with a ratio of $40 / 60(20 E / 20 Z)$.<smiles>CO/C(=C/c1c(OC)cc(OC)cc1OC)C(=O)C1=C(C)CCCC1</smiles>

E-2-(2-Methyl-cyclohex-1-enecarbonyl)-3-(2,4,6-trimethoxy-phenyl)-acrylic acid methyl ester (20E). Purified by flash chromatography (silica gel, ethyl acetate: hexanes $=2: 3) .{ }^{1} \mathrm{H}$ NMR $\left(400 \mathrm{MHz}, \mathrm{CDCl}_{3}\right): \delta 1.44(\mathrm{~m}, 4 \mathrm{H}), 1.89(\mathrm{~s}, 3 \mathrm{H}), 2.01(\mathrm{~m}, 2 \mathrm{H}), 2.12$ $(\mathrm{m}, 2 \mathrm{H}), 3.61(\mathrm{~s}, 6 \mathrm{H}), 3.71(\mathrm{~s}, 3 \mathrm{H}), 3.74(\mathrm{~s}, 3 \mathrm{H}), 6.00(\mathrm{~s}, 2 \mathrm{H}), 7.72(\mathrm{~s}, 1 \mathrm{H}) ;{ }^{13} \mathrm{C}$ NMR $\left(100 \mathrm{MHz}, \mathrm{CDCl}_{3}\right): \delta 22.0,22.3,22.5,26.5,34.2,51.8,54.8,55.2,55.3,90.2,105.1$, $131.8,132.5,134.6,145.7,159.1,162.9,166.9,195.5$; IR $\left(\mathrm{NaCl}\right.$ plate, $\left.\mathrm{cm}^{-1}\right): 2935,1726$, $1603,1460,1336,1233,1208,1158,1129$; Elemental analysis calculated for $\mathrm{C}_{19} \mathrm{H}_{22} \mathrm{O}_{7}$ : C $62.97 \%$; H $6.12 \%$. Found : C $62.79 \%$; H $6.20 \%$.

${ }^{3} \mathrm{~J}_{\mathrm{C}-\mathrm{H}}=9.7 \mathrm{~Hz}$ for $\mathrm{C}_{\text {ketone }}-\mathrm{H},{ }^{3} \mathrm{~J}_{\mathrm{C}-\mathrm{H}}=7.9 \mathrm{~Hz}$ for $\mathrm{C}_{\text {ester }}-\mathrm{H}$.

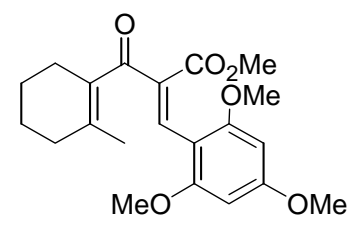

Z-2-(2-Methyl-cyclohex-1-enecarbonyl)-3-(2,4,6-trimethoxy-phenyl)-acrylic acid methyl ester (20Z). Purified by flash chromatography (silica gel, ethyl acetate: hexanes = 2:3). ${ }^{1} \mathrm{H}$ NMR (400 MHz, $\left.\mathrm{CDCl}_{3}\right): \delta 1.56(\mathrm{~m}, 4 \mathrm{H}), 1.59(\mathrm{~s}, 3 \mathrm{H}), 1.93(\mathrm{~m}, 2 \mathrm{H}), 2.13(\mathrm{~m}$, $2 \mathrm{H}), 3.60(\mathrm{~s}, 3 \mathrm{H}), 3.68(\mathrm{~s}, 6 \mathrm{H}), 3.73(\mathrm{~s}, 3 \mathrm{H}), 5.99(\mathrm{~s}, 2 \mathrm{H}), 7.65(\mathrm{~s}, 1 \mathrm{H}) ;{ }^{13} \mathrm{C}$ NMR $(100$ $\left.\mathrm{MHz}, \mathrm{CDCl}_{3}\right): \delta 20.8,22.0,22.4,27.2,31.0,51.2,55.2,55.3,90.2,104.9,132.3,134.2$, 137.3, 159.7, 163.8, 167.5, 199.4; IR (NaCl plate, $\left.\mathrm{cm}^{-1}\right): 2935,1726,1603,1460,1336$, 1233, 1208, 1158, 1129; HRMS calculated for $\mathrm{C}_{21} \mathrm{H}_{26} \mathrm{O}_{6}(\mathrm{M}+\mathrm{H})^{+}: 375.4308$, Found: 375.43063 .

${ }^{3} \mathrm{~J}_{\mathrm{C}-\mathrm{H}}=7.0 \mathrm{~Hz}$ for $\mathrm{C}_{\text {ketone }}-\mathrm{H},{ }^{3} \mathrm{~J}_{\mathrm{C}-\mathrm{H}}=12.0 \mathrm{~Hz}$ for $\mathrm{C}_{\text {ester }}-\mathrm{H}$. 

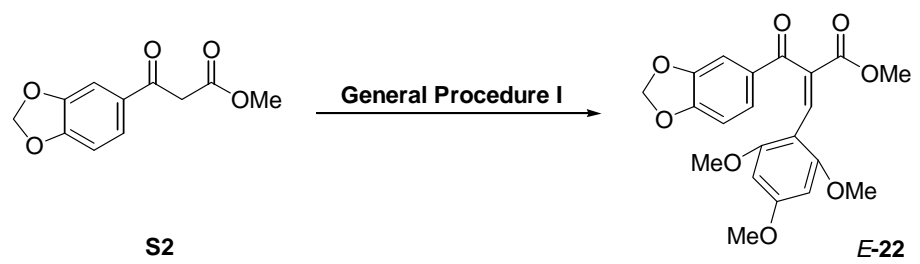

Purified by flash chromatography (silica gel, ethyl acetate: hexanes $=1: 1)$ to give 22 in $53 \%$ yield ${ }^{1} \mathbf{H}$ NMR $\left(\mathbf{4 0 0} \mathbf{~ M H z}, \mathbf{C D C l}_{3}\right) \delta 8.15(\mathrm{~s}, 1 \mathrm{H}), 7.48(\mathrm{q}, J=9.8 \mathrm{~Hz}, 1 \mathrm{H}), 7.44(\mathrm{~d}$, $J=1.6 \mathrm{~Hz}, 1 \mathrm{H}), 6.79(\mathrm{~d}, J=8.1 \mathrm{~Hz}, 1 \mathrm{H}), 6.03(\mathrm{~s}, 2 \mathrm{H}), 5.96(\mathrm{~s}, 2 \mathrm{H}), 3.79(\mathrm{~s}, 3 \mathrm{H}), 3.72(\mathrm{~s}$, 3H), $3.53(\mathrm{~s}, 6 \mathrm{H}) ;{ }^{13} \mathbf{C}$ NMR (CDCl 3 , $\left.100 \mathrm{MHz}\right) \delta 191.8$ 167.2, 163.4, 159.6, 151.0, 147.6, 135.5, 132.5, 128.0, 125.1, 108.2, 107.5, 105.0, 101.5, 90.7, 55.2, 54.6, 52.0; IR (NaCl plate, $\mathbf{c m}^{-1}$ ): 1707, 1664, 1603, 1439, 1254, 1209, 1158, 1129, 1035; Elemental analysis calculated for $\mathrm{C}_{21} \mathrm{H}_{20} \mathrm{O}_{8}$ : $\mathrm{C} 63.00 \%$; $\mathrm{H}$ 5.03\%. Found : $\mathrm{C} 63.24 \%$; $\mathrm{H} 4.99 \%$.
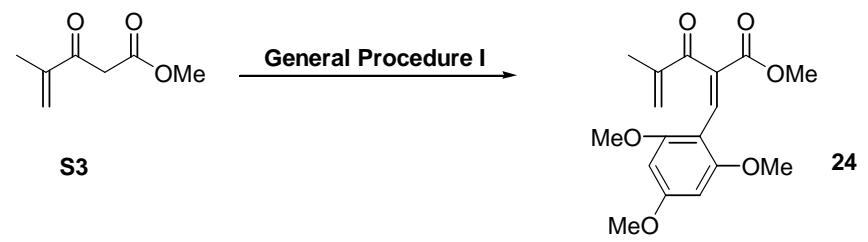

Purified by flash chromatography (silica gel, ethyl acetate: hexanes $=1: 2$ ) to obtain compound 24 in $74 \%$ yield. ${ }^{1} \mathbf{H}$ NMR (400 $\left.\mathbf{~ M H z}, \mathbf{C D C l}_{3}\right) \delta 7.95(\mathrm{~s}, 1 \mathrm{H}), 5.96(\mathrm{~s}, 2 \mathrm{H})$, $5.71(\mathrm{~s}, 1 \mathrm{H}), 5.61(\mathrm{~s}, 1 \mathrm{H}), 3.76(\mathrm{~s}, 3 \mathrm{H}), 3.71(\mathrm{~s}, 3 \mathrm{H}), 3.65(\mathrm{~s}, 6 \mathrm{H}), 1.85(\mathrm{~s}, 3 \mathrm{H}) ;{ }^{13} \mathbf{C}$ NMR $\left(\mathbf{C D C l}_{3}, \mathbf{1 0 0} \mathbf{M H z}\right) \delta 195.2,167.2,163.4,159.5,143.6,135.0,127.8,125.8,105.8,90.1$, 55.2, 54.9, 51.9, 16.8; IR (NaCl plate, $\mathbf{~ c m}^{-1}$ ): 1710, 1664, 1603, 1462, 1436, 1252, 1208, $1158,1129,735$; Elemental analysis calculated for $\mathrm{C}_{17} \mathrm{H}_{20} \mathrm{O}_{6}$ : C 63.74\%; $\mathrm{H} 6.29 \%$.

Found : C $63.86 \%$; H $6.35 \%$.

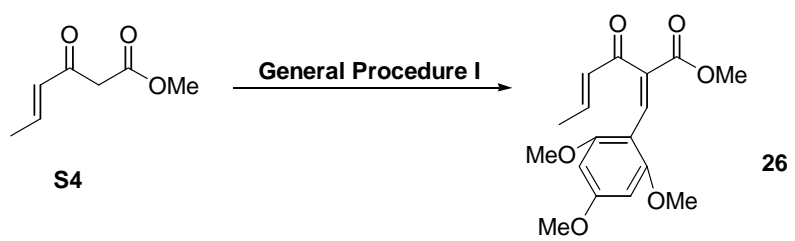

Purified by flash chromatography (silica gel, ethyl acetate: hexanes $=1: 1)$ to obtain compound 26 as an unseparable mixture regioisomers $(Z: E=0.6: 1.0)$ in $64 \%$ yield. ${ }^{1} \mathbf{H}$ NMR (400 MHz, $\left.\mathbf{C D C l}_{3}\right) \delta 7.95(\mathrm{~s}, 1 \mathrm{H}), 7.78(\mathrm{~s}, 0.6 \mathrm{H}), 7.00(\mathrm{~m}, 0.6 \mathrm{H}), 6.68(\mathrm{~m}, 1 \mathrm{H})$, $6.19(\mathrm{~d}, J=1.6 \mathrm{~Hz}, 0.6 \mathrm{H}), 6.15(\mathrm{~d}, J=1.6 \mathrm{~Hz}, 1 \mathrm{H}), 6.08(\mathrm{~s}, 1.2 \mathrm{H}), 6.04(\mathrm{~s}, 2 \mathrm{H}), 4.24(\mathrm{~m}$, $2 \mathrm{H}), 4.13(\mathrm{~m}, 1.2 \mathrm{H}), 3.88(\mathrm{~s}, 2.4 \mathrm{H}), 3.83(\mathrm{~s}, 3.6 \mathrm{H}), 3.79(\mathrm{~s}, 6 \mathrm{H}), 3.73(\mathrm{~s}, 6 \mathrm{H}), 1.92(\mathrm{~d}, J=$ $1.6 \mathrm{~Hz}, 1.2 \mathrm{H}), 1.82(\mathrm{~d}, J=1.8 \mathrm{~Hz}, 2 \mathrm{H}), 1.28(\mathrm{~m}, 1.8 \mathrm{H}), 1.24(\mathrm{~m}, 3 \mathrm{H}) ;{ }^{13} \mathbf{C} \mathbf{~ N M R}\left(\mathbf{C D C l}_{3}\right.$, 100 MHz) $\delta 193.0,188.3,167.8,166.5,163.5,163.3,159.8,159.5,143.7,142.4,136.1$, 135.1, 133.0, 132.0, 129.6, 128.6, 105.8, 105.2, 90.2, 60.9, 60.4, 55.4, 55.3, 54.9, 18.4, 18.1, 14.2, 13.9; IR (NaCl plate, $\mathbf{~ c m}^{-1}$ ): 1653, 1605, 1207, 1155, 1128, 500; Elemental analysis calculated for $\mathrm{C}_{18} \mathrm{H}_{22} \mathrm{O}_{6}$ : $\mathrm{C} 64.66 \% ; \mathrm{H} 6.63 \%$. Found : $\mathrm{C} 64.75 \%$; $\mathrm{H} 6.52 \%$.

The following compounds were prepared by Nazarov cyclization following General Procedure II: 

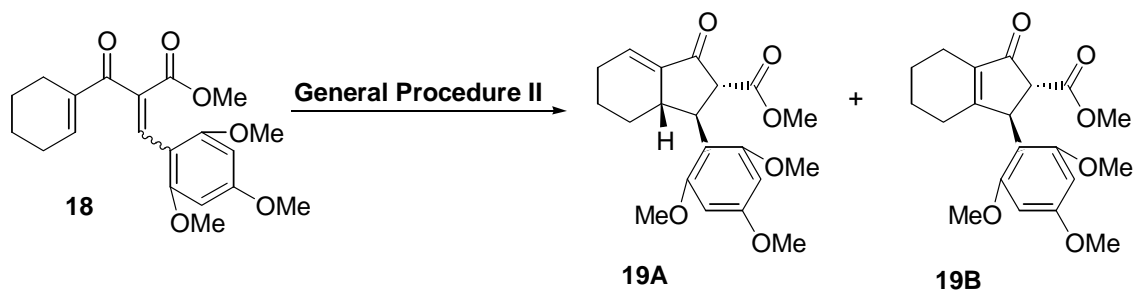

19A and 19B was obtained on Nazarov cyclization on 18 following General

Procedure II. Purified by flash chromatography (silica gel, ethyl acetate: hexanes $=1: 2$ )

Compound 19A was obtained in $39 \%$ yield. ${ }^{1} \mathbf{H}$ NMR (400 $\left.\mathbf{~ M H z}, \mathbf{C D C l}_{3}\right) \delta 6.80$ (dd, $\left.J_{1}=6.4 \mathrm{~Hz}, J_{2}=3.2 \mathrm{~Hz}, 1 \mathrm{H}\right), 6.14(\mathrm{~s}, 2 \mathrm{H}), 4.30(\mathrm{~d}, J=12.1 \mathrm{~Hz}, 1 \mathrm{H}), 3.80(\mathrm{t}, J=12.1$ $\mathrm{Hz}, 1 \mathrm{H}), 3.77(\mathrm{~s}, 3 \mathrm{H}), 3.74(\mathrm{~s}, 6 \mathrm{H}), 3.65(\mathrm{~s}, 3 \mathrm{H}), 2.93(\mathrm{~m}, 1 \mathrm{H}), 2.04(\mathrm{~m}, 2 \mathrm{H}), 1.80(\mathrm{~m}$, 2H), $1.25(\mathrm{~m}, 1 \mathrm{H}), 1.13(\mathrm{~m}, 1 \mathrm{H}) ;{ }^{13} \mathbf{C}$ NMR (CDCl 3 , $\left.100 \mathbf{~ M H z}\right) \delta 199.4,170.3,159.9$, 159.7, 140.6, 134.4, 107.5, 91.1, 91.0, 57.6, 55.2, 55.0, 52.0, 40.4, 39.6, 27.1, 25.6, 21.5; IR (NaCl plate, $\mathbf{~ c m}^{-1}$ ): $1742,1711,1606,1459,1202,1151,1115$; Elemental analysis calculated for $\mathrm{C}_{20} \mathrm{H}_{24} \mathrm{O}_{6}$ : C $66.65 \% ; \mathrm{H}, 6.71 \%$. Found : C $66.80 \% ; \mathrm{H} 6.75 \%$.

Compound 19B was obtained in $44 \%$ yield. ${ }^{1} \mathbf{H}$ NMR (400 MHz, $\left.\mathbf{C D C l}_{3}\right) \delta 6.16(\mathrm{~d}$, $J=2.4 \mathrm{~Hz}, 1 \mathrm{H}), 6.06(\mathrm{~d}, J=2.4 \mathrm{~Hz}, 1 \mathrm{H}), 4.84(\mathrm{~m}, 1 \mathrm{H}), 3.81(\mathrm{~s}, 6 \mathrm{H}), 3.74(\mathrm{~s}, 3 \mathrm{H}), 3.67$ $(\mathrm{s}, 3 \mathrm{H}), 3.59(\mathrm{~d}, J=5.8 \mathrm{~Hz}, 1 \mathrm{H}), 2.00(\mathrm{~m}, 2 \mathrm{H}), 1.73(\mathrm{~m}, 1 \mathrm{H}), 1.71(\mathrm{~m}, 1 \mathrm{H}), 1.63(\mathrm{~m}, 4 \mathrm{H})$; ${ }^{13}{ }^{13}$ NMR (CDCl 3 , 100 MHz) $\delta$ 201.6, 177.1, 170.7, 160.4, 159.4, 159.2, 134.9, 107.2, 90.7, 58.2, 56.0, 55.2, 55.1, 41.6, 26.2, 22.1, 21.5, 20.2; IR (NaCl plate, $\mathbf{~ c m}^{-1}$ ): 2937, 1737, 1697, 1643, 1604, 1459, 1436, 1332, 1258, 1228, 1205, 1150, 1116, 733;

Elemental analysis calculated for $\mathrm{C}_{20} \mathrm{H}_{24} \mathrm{O}_{6}$ : $\mathrm{C} 66.65 \% ; \mathrm{H}, 6.71 \%$. Found : $\mathrm{C} 66.81 \% ; \mathrm{H}$ $6.62 \%$.

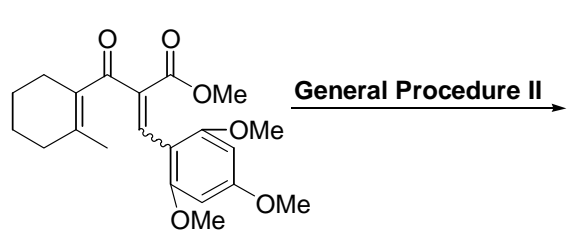

20

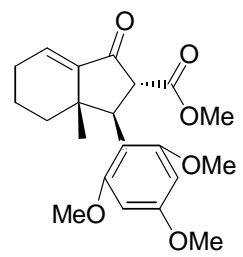

21

Compound $\mathbf{2 1}$ was obtained in 95\% yield from Nazarov cyclization of $\mathbf{2 0}$ following General Procedure II. Purified by flash chromatography (silica gel, ethyl acetate: hexanes $=1: 1)$.

${ }^{1} \mathrm{H}$ NMR (400 MHz, CDCl $) \delta 6.74(\mathrm{t}, J=4 \mathrm{~Hz}, 1 \mathrm{H}), 6.15(\mathrm{~s}, 2 \mathrm{H}), 4.90(\mathrm{~d}, J=13.2$ $\mathrm{Hz}, 1 \mathrm{H}), 4.05(\mathrm{~d}, J=13.2 \mathrm{~Hz}, 1 \mathrm{H}), 3.81(\mathrm{~s}, 3 \mathrm{H}), 3.77(\mathrm{~s}, 6 \mathrm{H}), 3.66(\mathrm{~s}, 3 \mathrm{H}), 2.21(\mathrm{~m}, 2 \mathrm{H})$, 1.63-1.71 (m, 4H), 1.24 (s. 3H); ${ }^{13} \mathbf{C}$ NMR (CDCl 3 , 100 MHz): $\delta$ 200.0, 170.6, 159.9, 144.8, 134.4, 105.9, 91.5, 55.1, 52.1, 45.6, 41.9, 34.1, 25.5, 20.6, 18.1; IR (NaCl plate, $\left.\mathbf{c m}^{-1}\right): 1740,1707,1646,1606,1459,1203,1152,1115$.
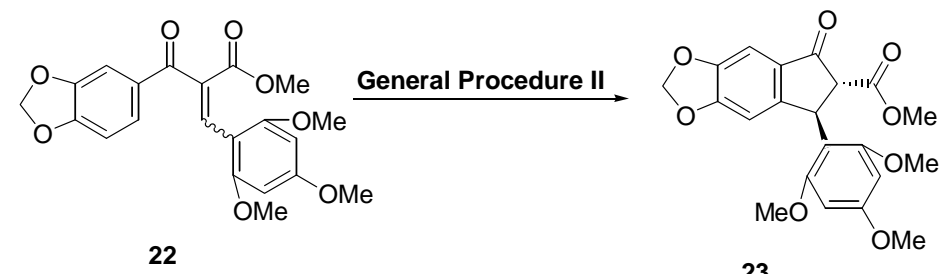

23

Compound 23 was obtained in 92\% yield on Nazarov cyclization on 22 following General Procedure II. Purified by flash chromatography (silica gel, ethyl acetate: hexanes $=1: 1)$. 
${ }^{1}$ H NMR (400 MHz, CDCl $\left._{3}\right) \delta 7.11(\mathrm{~s}, 1 \mathrm{H}), 6.55(\mathrm{~s}, 1 \mathrm{H}), 6.21(\mathrm{~s}, 1 \mathrm{H}), 6.03(\mathrm{~s}, 3 \mathrm{H})$, $5.42(\mathrm{~d}, J=3.6 \mathrm{~Hz}, 1 \mathrm{H}), 4.00(\mathrm{~d}, J=3.6 \mathrm{~Hz}, 1 \mathrm{H}), 3.89(\mathrm{~s}, 3 \mathrm{H}), 3.82(\mathrm{~s}, 3 \mathrm{H}), 3.77(\mathrm{~s}, 3 \mathrm{H})$, 3.47 (s, 3H); ${ }^{13}$ C NMR (CDCl 3 , 100 MHz) $\delta$ 197.9, 170.2, 160.5, 156.2, 154.3, 147.9, 129.0, 109.0, 104.4, 102.0, 91.1, 90.6, 59.8, 56.0, 55.2, 55.1, 52.3, 37.6; IR (NaCl plate, $\mathbf{c m}^{-1}$ ): 1738, 1700, 1607, 1497, 1467, 1329, 1294, 1248, 1205, 1151, 1118, 1035, 815, 734; Elemental analysis calculated for $\mathrm{C}_{21} \mathrm{H}_{20} \mathrm{O}_{8}$ : $\mathrm{C} 63.00 \%$; H 5.03\%. Found : $\mathrm{C}$ $62.99 \%$; H $5.22 \%$.

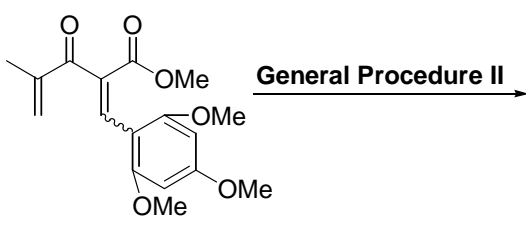

24

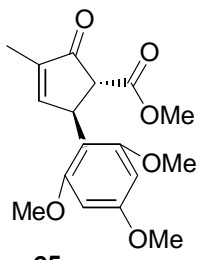

25

Compound 25 was obtained in $75 \%$ yield on Nazarov cyclization on $\mathbf{2 4}$ following General Procedure II. Purified by flash chromatography (silica gel, ethyl acetate: hexanes $=1: 2$ ).

${ }^{1} \mathbf{H}$ NMR (400 MHz, $\left.\mathbf{C D C l}_{3}\right) \delta 7.20(\mathrm{t}, J=1.2 \mathrm{~Hz}, 1 \mathrm{H}), 6.08(\mathrm{~s}, 2 \mathrm{H}), 4.90(\mathrm{q}, J=$ $2.4 \mathrm{~Hz}, 1 \mathrm{H}), 3.76(\mathrm{~s}, 3 \mathrm{H}), 3.73(\mathrm{~s}, 3 \mathrm{H}), 3.71(\mathrm{~s}, 6 \mathrm{H}), 3.63(\mathrm{~d}, J=2.4 \mathrm{~Hz}, 1 \mathrm{H}), 1.80(\mathrm{~s}, 3 \mathrm{H})$; ${ }^{13} \mathbf{C}$ NMR (CDCl 3 , 100 MHz) $\delta$ 203.6, 170.2, 161.6, 160.4, 159.1, 137.3, 107.2, 90.7, 57.4, 55.5, 55.2, 52.2, 38.4, 9.9; IR (NaCl plate, $\mathbf{~ c m}^{-1}$ ): 1738, 1704, 1636, 1605, 1459, $1436,1333,1256,1221,1204,1151,1119$; Elemental analysis calculated for $\mathrm{C}_{17} \mathrm{H}_{20} \mathrm{O}_{6}$. C 63.74\%; H 6.29\%. Found : C 63.57\%; H 6.13\%

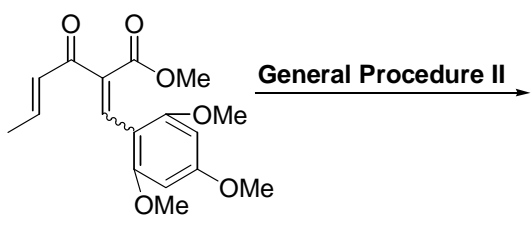

26

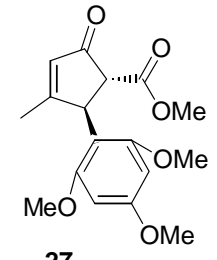

27

Compound $\mathbf{2 7}$ was obtained in 58\% yield on Nazarov cyclization on $\mathbf{2 6}$ following General Procedure II. Purified by flash chromatography (silica gel, ethyl acetate: hexanes $=1: 2$ ).

${ }^{1}$ H NMR (400 MHz, $\left.\mathbf{C D C l}_{3}\right) \delta 6.15(\mathrm{~d}, J=2.1 \mathrm{~Hz}, 1 \mathrm{H}), 6.06(\mathrm{~s}, 1 \mathrm{H}), 5.92(\mathrm{~s}, 1 \mathrm{H})$, 4.92 (br. s, $1 \mathrm{H}), 4.18$ (m, 2H), 3.80 (s, 6H), 3.63 (m, 4H), 1.88 (s, 3H), 1.25 (t, $J=7.2$ $\mathrm{Hz}, 3 \mathrm{H}) ;{ }^{13} \mathbf{C}$ NMR $\left(\mathrm{CDCl}_{3}, 100 \mathrm{MHz}\right) \delta 202.6,182.4,169.9,160.6,159.6,159.2,127.0$, 106.4, 90.7, 90.5, 61.2, 59.1, 56.0, 55.3, 55.2, 43.8, 17.3, 14.2; IR (NaCl plate, $\mathbf{~ c m}^{-1}$ ): 2939, 1732, 1700, 1606, 1461, 1420, 1331, 1205, 1150, 1120, 1058, 1035, 815;

Elemental analysis calculated for $\mathrm{C}_{18} \mathrm{H}_{22} \mathrm{O}_{6}$ : $\mathrm{C} 64.66 \% ; \mathrm{H} 6.63 \%$. Found : $\mathrm{C} 64.75 \% ; \mathrm{H}$ $6.52 \%$. 


\section{Preparation of Compounds in Table 3}

\section{The following compounds were prepared by Knoevenagel condensation following General Procedure I:}

Knoevenagel condensation following General Procedure I gave a mixture of isomers with a ratio of $62 / 28(\mathbf{2 8 E} / \mathbf{2 8 Z})$.<smiles>COC(=O)C(=Cc1ccc(OC)cc1)C(=O)C1=C(C)CCCC1</smiles>

E-3-(4-Methoxy-phenyl)-2-(2-methyl-cyclohex-1-enecarbonyl)-acrylic acid methyl ester (28E). Purified by gravity chromatography (silica gel, ethyl acetate: hexanes $=1: 6)$. ${ }^{1} \mathrm{H}$ NMR $\left(400 \mathrm{MHz}, \mathrm{CDCl}_{3}\right): \delta 1.46(\mathrm{~m}, 4 \mathrm{H}), 2.01(\mathrm{~s}, 3 \mathrm{H}), 2.09(\mathrm{~m}, 2 \mathrm{H}), 2.16(\mathrm{~m}, 2 \mathrm{H})$, $3.77(\mathrm{~s}, 6 \mathrm{H}), 6.78(\mathrm{~d}, 2 \mathrm{H}, \mathrm{J}=8.8 \mathrm{~Hz}), 7.25(\mathrm{~d}, 2 \mathrm{H}, \mathrm{J}=8.8 \mathrm{~Hz}), 7.58(\mathrm{~s}, 1 \mathrm{H}) ;{ }^{13} \mathrm{C} \mathrm{NMR}$ $\left(100 \mathrm{MHz}, \mathrm{CDCl}_{3}\right): \delta 21.9,22.2,22.4,26.2,34.6,52.1,55.1,113.9,125.8,131.5,131.5$, 131.6, 140.0, 150.0, 161.0, 165.8, 197.5; IR (NaCl plate, $\left.\mathrm{cm}^{-1}\right): 2933,2858,2840,1716$, $1600,1512,1436,1305,1265,1174,1029,833,736$; HRMS calculated for $\mathrm{C}_{19} \mathrm{H}_{22} \mathrm{O}_{4}$ $(\mathrm{M}+\mathrm{H})^{+}: 315.1591$, Found: 315.15842 .

${ }^{3} \mathrm{~J}_{\mathrm{C}-\mathrm{H}}=9.5 \mathrm{~Hz}$ for $\mathrm{C}_{\mathrm{ketone}}-\mathrm{H},{ }^{3} \mathrm{~J}_{\mathrm{C}-\mathrm{H}}=8.1 \mathrm{~Hz}$ for $\mathrm{C}_{\mathrm{ester}}-\mathrm{H}$.<smiles>COc1ccc(/C=C(\C(C)=O)C(=O)C2=C(C)CCCC2)cc1</smiles>

Z-3-(4-Methoxy-phenyl)-2-(2-methyl-cyclohex-1-enecarbonyl)-acrylic acid methyl ester (28Z). Purified by gravity chromatography (silica gel, ethyl acetate: hexanes $=1: 6$ ). ${ }^{1} \mathrm{H}$ NMR (400 MHz, $\left.\mathrm{CDCl}_{3}\right): \delta 1.68(\mathrm{~m}, 7 \mathrm{H}), 2.07(\mathrm{~m}, 2 \mathrm{H}), 2.22(\mathrm{~m}, 2 \mathrm{H}), 3.84(\mathrm{~s}, 3 \mathrm{H})$, $3.87(\mathrm{~s}, 3 \mathrm{H}), 6.92(\mathrm{~d}, 2 \mathrm{H}, \mathrm{J}=8.8 \mathrm{~Hz}), 7.39(\mathrm{~s}, 1 \mathrm{H}), 7.43(\mathrm{~d}, 2 \mathrm{H}, \mathrm{J}=8.8 \mathrm{~Hz}) ;{ }^{13} \mathrm{C} \mathrm{NMR}$ $\left(100 \mathrm{MHz}, \mathrm{CDCl}_{3}\right): \delta 20.9,22.0,22.4,27.2,30.9,52.3,55.3,113.9,125.3,131.4,131.5$, 131.6, 134.8, 143.0, 161.7, 168.3, 198.8; IR ( $\mathrm{NaCl}$ plate, $\left.\mathrm{cm}^{-1}\right): 2933,2836,1720,1606$, 1436, 1300, 1170, 1015, 831, 736; HRMS calculated for $\mathrm{C}_{19} \mathrm{H}_{22} \mathrm{O}_{4}(\mathrm{M}+\mathrm{H})^{+}: 315.1591$, Found: 315.15900.

${ }^{3} \mathrm{~J}_{\mathrm{C}-\mathrm{H}}=6.7 \mathrm{~Hz}$ for $\mathrm{C}_{\mathrm{ketone}}-\mathrm{H},{ }^{3} \mathrm{~J}_{\mathrm{C}-\mathrm{H}}=12.5 \mathrm{~Hz}$ for $\mathrm{C}_{\text {ester }}-\mathrm{H}$.<smiles>CC(=O)C(=Cc1ccccc1)C(=O)C1=C(C)CCCC1</smiles>

2-(2-Methyl-cyclohex-1-enecarbonyl)-3-phenyl-acrylic acid methyl ester (30E/Z).

Knoevenagel condensation following General Procedure I gave a mixture of isomers with a ratio of $71 / 39(30 E / 30 Z)$, which purified by flash chromatography (silica gel, ethyl acetate: hexanes $=1: 6)$ to get an analytical sample. ${ }^{1} \mathrm{H}$ NMR $\left(400 \mathrm{MHz}, \mathrm{CDCl}_{3}\right): \delta 1.29$ (m, 2.7 H), $1.52(\mathrm{~m}, 2.3 \mathrm{H}), 1.90(\mathrm{~m}, 4 \mathrm{H}), 2.05(\mathrm{~m}, 2 \mathrm{H}), 3.63(\mathrm{~s}, 3 \mathrm{H}), 7.19(\mathrm{~d}, 3.3 \mathrm{H})$, 
7.30 (s, $2 \mathrm{H}), 7.54(\mathrm{~d}, 0.7 \mathrm{H}) ;{ }^{13} \mathrm{C} \mathrm{NMR}\left(100 \mathrm{MHz}, \mathrm{CDCl}_{3}\right): \delta 20.9,21.8,22.0,22.1,22.3$, $22.4,26.2,27.1,30.9,34.5,52.2,128.4,128.7,129.4,129.5,129.9,130.6,131.2,131.4$, 132.9, 133.3, 134.2, 134.3, 135.5, 140.3, 143.0, 150.1, 165.6, 167.7, 196.9, 198.6; IR ( $\mathrm{NaCl}$ plate, $\left.\mathrm{cm}^{-1}\right): 2936,2859,1721,1665,1619,1448,1435,1376,1254,1200,466$; HRMS calculated for $\mathrm{C}_{18} \mathrm{H}_{20} \mathrm{O}_{3}(\mathrm{M}+\mathrm{H})^{+}: 285.1485$, Found: 285.14832 .

$\boldsymbol{E}^{3} \mathrm{~J}_{\mathrm{C}-\mathrm{H}}=9.2 \mathrm{~Hz}$ for $\mathrm{C}_{\text {ketone }}-\mathrm{H},{ }^{3} \mathrm{~J}_{\mathrm{C}-\mathrm{H}}=7.9 \mathrm{~Hz}$ for $\mathrm{C}_{\text {ester }}-\mathrm{H}$.

$\mathrm{Z}^{3} \mathrm{~J}_{\mathrm{C}-\mathrm{H}}=7.2 \mathrm{~Hz}$ for $\mathrm{C}_{\text {ketone }}-\mathrm{H},{ }^{3} \mathrm{~J}_{\mathrm{C}-\mathrm{H}}=12.6 \mathrm{~Hz}$ for $\mathrm{C}_{\text {ester }}-\mathrm{H}$.<smiles>COc1ccc([N+](=O)[O-])cc1</smiles>

E-3-(4-Nitrophenyl)-2-(2-methyl-cyclohex-1-enecarbonyl)-acrylic acid methyl ester (32E). Purified by gravity chromatography (silica gel, ethyl acetate: hexanes $=1: 6$ ). ${ }^{1} \mathrm{H}$ NMR (400 MHz, $\left.\mathrm{CDCl}_{3}\right): \delta 1.50(\mathrm{~m}, 4 \mathrm{H}), 2.03(\mathrm{~s}, 3 \mathrm{H}), 2.12(\mathrm{~m}, 2 \mathrm{H}), 3.83(\mathrm{~s}, 3 \mathrm{H}) 7.46$ $(\mathrm{d}, \mathrm{J}=8.8 \mathrm{~Hz}, 2 \mathrm{H}), 7.67(\mathrm{~s}, 1 \mathrm{H}) 8.16(\mathrm{~d}, \mathrm{~J}=8.8 \mathrm{~Hz}, 2 \mathrm{H}) ;{ }^{13} \mathrm{C} \mathrm{NMR}\left(100 \mathrm{MHz}, \mathrm{CDCl}_{3}\right)$ : $\delta 21.8,22.1,22.6,26.3,34.7,52.7,123.6,130.2,131.1,137.1,138.0,139.7,148.0,151.9$, 164.9, 195.7; IR ( NaCl plate, $\left.\mathrm{cm}^{-1}\right): 3080,3060,2840,1718,1665,1524,1355,1252$, 1107, 1030, 802, 724; HRMS calculated for $\mathrm{C}_{18} \mathrm{H}_{20} \mathrm{NO}_{5}(\mathrm{M}+\mathrm{H})^{+}$: 330.1341 , Found: 330.1340 .

${ }^{3} \mathrm{~J}_{\mathrm{C}-\mathrm{H}}=9.0 \mathrm{~Hz}$ for $\mathrm{C}_{\text {ketone }}-\mathrm{H},{ }^{3} \mathrm{~J}_{\mathrm{C}-\mathrm{H}}=7.9 \mathrm{~Hz}$ for $\mathrm{C}_{\text {ester }}-\mathrm{H}$.

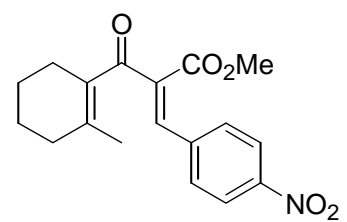

Z-3-(4-Nitrophenyl)-2-(2-methyl-cyclohex-1-enecarbonyl)-acrylic acid methyl ester (32Z). Purified by gravity chromatography (silica gel, ethyl acetate: hexanes $=1: 6$ ). ${ }^{1} \mathrm{H}$ NMR (400 MHz, CDCl $)$ ): $\delta 1.67$ (m, $5 \mathrm{H}), 2.08$ (br s, 3H) 2.21 (br s, $3 \mathrm{H}), 3.81(\mathrm{~s}, 3 \mathrm{H})$, $7.42(\mathrm{~s}, 1 \mathrm{H}), 7.57(\mathrm{~d}, 2 \mathrm{H}, \mathrm{J}=8.8 \mathrm{~Hz}), 8.21(\mathrm{~d}, 2 \mathrm{H}, \mathrm{J}=8.8 \mathrm{~Hz}) ;{ }^{13} \mathrm{C} \mathrm{NMR}(100 \mathrm{MHz}$, $\left.\mathrm{CDCl}_{3}\right): \delta 21.3,22.1,22.4,27.2,31.3,52.7,123.7,130.0,131.2,137.5,137.7,139.5$, 139.6, 148.5, 166.8, 197.6; IR ( NaCl plate, $\left.\mathrm{cm}^{-1}\right): 3102,3020,2844,1718,1666,1518$, $1347,1254,1117,1042,877,802,721$; HRMS calculated for $\mathrm{C}_{18} \mathrm{H}_{20} \mathrm{NO}_{5}(\mathrm{M}+\mathrm{H})^{+}$: 330.1341, Found: 330.1340.

${ }^{3} \mathrm{~J}_{\mathrm{C}-\mathrm{H}}=6.5 \mathrm{~Hz}$ for $\mathrm{C}_{\text {ketone }}-\mathrm{H},{ }^{3} \mathrm{~J}_{\mathrm{C}-\mathrm{H}}=12.4 \mathrm{~Hz}$ for $\mathrm{C}_{\text {ester }}-\mathrm{H}$.

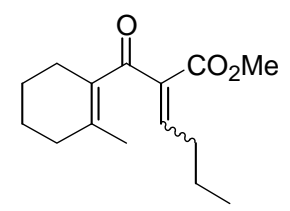

2-(2-Methyl-cyclohex-1-enecarbonyl)-hex-2-enoic acid methyl ester (34E/Z).

Knoevenagel condensation following General Procedure I gave a mixture of isomers with a ratio of of $49 / 51(34 E / 34 Z)$, which purified by flash chromatography (silica gel, ethyl acetate: hexanes $=1: 19)$ to get an analytical sample. ${ }^{1} \mathrm{H}$ NMR $\left(400 \mathrm{MHz}, \mathrm{CDCl}_{3}\right): \delta 0.87$ 
(m, $3 \mathrm{H}), 1.45(\mathrm{~m}, 2 \mathrm{H}), 1.54(\mathrm{~s}, 7 \mathrm{H}), 1.89(\mathrm{~m}, 1 \mathrm{H}), 2.08(\mathrm{~m}, 4 \mathrm{H}), 2.27$ (t, $1 \mathrm{H}, \mathrm{J}=7.4$ $\mathrm{Hz}), 3.67(\mathrm{~s}, 1.5 \mathrm{H}), 2.74(\mathrm{~s}, 1.5 \mathrm{H}), 6.64(\mathrm{t}, 0.51 \mathrm{H}, \mathrm{J}=7.9 \mathrm{~Hz}), 6.85$ (t, $0.49 \mathrm{H}, \mathrm{J}=7.9$ $\mathrm{Hz}) ;{ }^{13} \mathrm{C} \mathrm{NMR}\left(100 \mathrm{MHz}, \mathrm{CDCl}_{3}\right): \delta$ 13.5, 13.6, 20.8, 21.7, 22.0 22.0, 22.0, 22.1, 22.2, $22.2,26.2,27.0,30.1,31.1,31.7,33.8,51.7,51.7,131.6,132.4,135.9,136.0,136.9$, 146.3, 146.5, 149.9, 165.1, 166.4, 196.9, 198.1; IR ( $\mathrm{NaCl}$ plate, $\left.\mathrm{cm}^{-1}\right): 2932,2864,1726$, $1669,1643,1436,1282,1247,1222,471$; HRMS calculated for $\mathrm{C}_{15} \mathrm{H}_{22} \mathrm{O}_{3}(\mathrm{M}+\mathrm{Na})^{+}$: 273.1461, Found: 273.14645 .

$\boldsymbol{E}^{3} \mathrm{~J}_{\mathrm{C}-\mathrm{H}}=9.2 \mathrm{~Hz}$ for $\mathrm{C}_{\text {ketone }}-\mathrm{H},{ }^{3} \mathrm{~J}_{\mathrm{C}-\mathrm{H}}=7.3 \mathrm{~Hz}$ for $\mathrm{C}_{\text {ester }}-\mathrm{H}$.

$Z^{3} \mathrm{~J}_{\mathrm{C}-\mathrm{H}}=6.8 \mathrm{~Hz}$ for $\mathrm{C}_{\text {ketone }}-\mathrm{H},{ }^{3} \mathrm{~J}_{\mathrm{C}-\mathrm{H}}=12.6 \mathrm{~Hz}$ for $\mathrm{C}_{\text {ester }}-\mathrm{H}$.

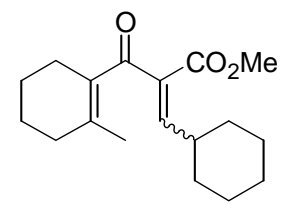

3-Cyclohexyl-2-(2-methyl-cyclohex-1-enecarbonyl)-acrylic acid methyl ester (36E/Z). Knoevenagel condensation following General Procedure I gave a mixture of isomers with a ratio of 57/43 (36E/36Z), which purified by flash chromatography (silica gel, ethyl acetate: hexanes $=1: 6)$ to get an analytical sample. ${ }^{1} \mathrm{H}$ NMR $(400 \mathrm{MHz}$, $\left.\mathrm{CDCl}_{3}\right): \delta 1.19(\mathrm{~m}, 5 \mathrm{H}), 1.63(\mathrm{~m}, 10 \mathrm{H}), 1.94(\mathrm{~s}, 1.6 \mathrm{H}), 2.15(\mathrm{~m}, 1 \mathrm{H}), 2.22(\mathrm{~m}, 4 \mathrm{H})$, $2.49(\mathrm{~m}, 0.4 \mathrm{H}), 7.77(\mathrm{~s}, 3 \mathrm{H}), 7.48(\mathrm{~d}, 0.47 \mathrm{H}, \mathrm{J}=10.7 \mathrm{~Hz}), 6.72(\mathrm{~d}, 0.53 \mathrm{H}, \mathrm{J}=10.7 \mathrm{~Hz})$; ${ }^{13} \mathrm{C}$ NMR $\left(100 \mathrm{MHz}, \mathrm{CDCl}_{3}\right): \delta 21.0,21.8,22.0,22.0,22.2,22.2,24.9,25.0,25.4,26.3$, 27.0, 31.2, 31.4, 31.5, 33.7, 38.3, 38.8, 51.7, 51.8, 131.6, 132.6, 134.1, 134.8, 136.2, 146.0, 151.1 154.2, 165.4, 166.6, 197.1, 198.3; IR $\left(\mathrm{NaCl}\right.$ plate, $\left.\mathrm{cm}^{-1}\right): 2929,2853,1727$, $1689,1668,1448,1437,1274,1255,1227,459$; HRMS calculated for $\mathrm{C}_{18} \mathrm{H}_{26} \mathrm{O}_{3}(\mathrm{M}+\mathrm{H})^{+}$: 291.1955, Found: 291.19687.

$\boldsymbol{E}^{3} \mathrm{~J}_{\mathrm{C}-\mathrm{H}}=9.2 \mathrm{~Hz}$ for $\mathrm{C}_{\text {ketone }}-\mathrm{H},{ }^{3} \mathrm{~J}_{\mathrm{C}-\mathrm{H}}=7.4 \mathrm{~Hz}$ for $\mathrm{C}_{\text {ester }}-\mathrm{H}$.

$\mathrm{Z}^{3} \mathrm{~J}_{\mathrm{C}-\mathrm{H}}=6.4 \mathrm{~Hz}$ for $\mathrm{C}_{\text {ketone }}-\mathrm{H},{ }^{3} \mathrm{~J}_{\mathrm{C}-\mathrm{H}}=12.6 \mathrm{~Hz}$ for $\mathrm{C}_{\text {ester }}-\mathrm{H}$.

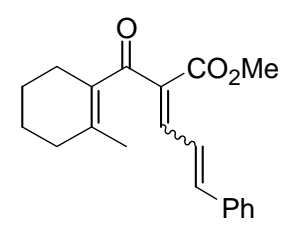

2-(2-Methyl-cyclohex-1-enecarbonyl)-5-phenyl-penta-2,4-dienoic acid methyl ester (37E/Z). Knoevenagel condensation following General Procedure I gave a mixture of isomers with a ratio of 57/43 (37E/37Z), which purified by flash chromatography (silica gel, ethyl acetate: hexanes $=1: 9)$ to get an analytical sample. ${ }^{1} \mathrm{H}$ NMR $(400 \mathrm{MHz}$, $\left.\mathrm{CDCl}_{3}\right): \delta 1.61(\mathrm{~m}, 4 \mathrm{H}), 1.67(\mathrm{~s}, 1.3 \mathrm{H}), 1.93(\mathrm{~s}, 1.7 \mathrm{H}), 2.12(\mathrm{~m}, 4 \mathrm{H}), 3.73(\mathrm{~s}, 1.7 \mathrm{H})$, $3.82(\mathrm{~s}, 1.3 \mathrm{H}), 6.99(\mathrm{~m}, 1.5 \mathrm{H}), 7.38(\mathrm{~m}, 6.5 \mathrm{H}) ;{ }^{13} \mathrm{C}$ NMR $\left(100 \mathrm{MHz}, \mathrm{CDCl}_{3}\right): \delta 21.0$, 21.7, 22.2, 22.3, 22.4, 26.5, 27.1, 31.5, 33.7, 51.9, 52.0, 123.0, 123.8, 127.5, 127.8, 128.7, $128.8,129.5,129.8,132.3,133.2,133.2,132.6,135.6,135.7,136.5,142.8,143.6,144.9$, 145.1, 145.3, 165.7, 166.4, 196.9, 197.8; IR (NaCl plate, $\left.\mathrm{cm}^{-1}\right): 2930,2859,1714,1689$, 1586, 1448, 1370, 1281, 1178, 978, 752, 691, 472; HRMS calculated for $\mathrm{C}_{20} \mathrm{H}_{22} \mathrm{O}_{3}$ $(\mathrm{M}+\mathrm{H})^{+}: 311.1642$, Found: 311.16412 .

$\boldsymbol{E}^{3} \mathrm{~J}_{\mathrm{C}-\mathrm{H}}=8.7 \mathrm{~Hz}$ for $\mathrm{C}_{\text {ketone }}-\mathrm{H},{ }^{3} \mathrm{~J}_{\mathrm{C}-\mathrm{H}}=6.6 \mathrm{~Hz}$ for $\mathrm{C}_{\text {ester }}-\mathrm{H}$.

$\mathrm{Z}^{3} \mathrm{~J}_{\mathrm{C}-\mathrm{H}}=5.4 \mathrm{~Hz}$ for $\mathrm{C}_{\text {ketone }}-\mathrm{H},{ }^{3} \mathrm{~J}_{\mathrm{C}-\mathrm{H}}=12.1 \mathrm{~Hz}$ for $\mathrm{C}_{\text {ester }}-\mathrm{H}$. 


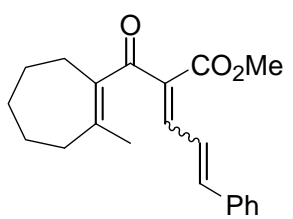

2-(2-Methyl-cyclohept-1-enecarbonyl)-5-phenyl-penta-2,4-dienoic acid methyl ester $(39 E / Z)$. Knoevenagel condensation following General Procedure I gave a mixture of isomers with a ratio of $51 / 49(39 E / 39 Z)$, which purified by flash chromatography (silica gel, ethyl acetate: hexanes $=1: 6)$ to get an analytical sample. ${ }^{1} \mathrm{H}$ NMR $(400 \mathrm{MHz}$, $\left.\mathrm{CDCl}_{3}\right): \delta 1.51(\mathrm{~m}, 4 \mathrm{H}), 1.72(\mathrm{~m}, 2 \mathrm{H}), 1.77(\mathrm{~s}, 1.54 \mathrm{H}), 2.02(\mathrm{~s}, 1.46 \mathrm{H}), 2.27(\mathrm{~m}, 3 \mathrm{H})$, $2.42(\mathrm{~s}, 1 \mathrm{H}), 3.74(\mathrm{~s}, 1.54 \mathrm{H}), 3.83(\mathrm{~s}, 1.46 \mathrm{H}), 7.02(\mathrm{~m}, 2 \mathrm{H}), 7.30(\mathrm{~m}, 6 \mathrm{H}) ;{ }^{13} \mathrm{C} \mathrm{NMR}$ $\left(100 \mathrm{MHz}, \mathrm{CDCl}_{3}\right): \delta 23.2,23.4,25.0,25.4,26.3,26.7,30.0,31.0,32.2,36.5,38.0,52.0$, 123.1, 123.7, 127.6, 127.8, 128.9, 129.6, 129.8, 133.2, 135.5, 135.6, 137.2, 139.1, 143.3, 143.7, 144.6, 144.8, 145.2, 152.0, 165.7, 166.4, 196.9, 197.8; IR ( $\mathrm{NaCl}$ plate, $\left.\mathrm{cm}^{-1}\right)$ : 2923, 2850, 1717, 1613, 1586, 1448, 1233, 1180, 982, 752, 691, 472; HRMS calculated for $\mathrm{C}_{21} \mathrm{H}_{24} \mathrm{O}_{3}(\mathrm{M}+\mathrm{H})^{+}:$325.1798, Found: 325.17990 .

$\boldsymbol{E}^{3} \mathrm{~J}_{\mathrm{C}-\mathrm{H}}=9.2 \mathrm{~Hz}$ for $\mathrm{C}_{\text {ketone }}-\mathrm{H},{ }^{3} \mathrm{~J}_{\mathrm{C}-\mathrm{H}}=6.7 \mathrm{~Hz}$ for $\mathrm{C}_{\text {ester }}-\mathrm{H}$.

$\mathrm{Z}^{3} \mathrm{~J}_{\mathrm{C}-\mathrm{H}}=4.6 \mathrm{~Hz}$ for $\mathrm{C}_{\text {ketone }}-\mathrm{H},{ }^{3} \mathrm{~J}_{\mathrm{C}-\mathrm{H}}=10.7 \mathrm{~Hz}$ for $\mathrm{C}_{\text {ester }}-\mathrm{H}$.

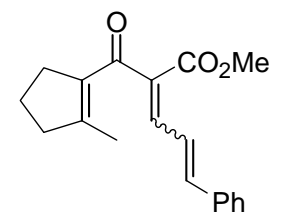

Z-2-(2-Methyl-cyclopent-1-enecarbonyl)-5-phenyl-penta-2,4-dienoic acid methyl ester $(41 E / Z)$. Knoevenagel condensation following General Procedure I gave a mixture of isomers with a ratio of $78 / 22(\mathbf{4 1 E} / \mathbf{4 1 Z})$, which purified by flash chromatography (silica gel, ethyl acetate: hexanes $=1: 4)$ to get an analytical sample. ${ }^{1} \mathrm{H}$ NMR $(400 \mathrm{MHz}$, $\left.\mathrm{CDCl}_{3}\right): \delta 1.84(\mathrm{~m}, 2 \mathrm{H}), 1.95(\mathrm{~s}, 0.7 \mathrm{H}), 2.04(\mathrm{~s}, 2.3 \mathrm{H}), 2.56(\mathrm{~m}, 2 \mathrm{H}), 2.68(\mathrm{~m}, 2 \mathrm{H})$, 3.79 (s, $2.3 \mathrm{H}), 3.83(\mathrm{~s}, 0.7 \mathrm{H}), 6.84(\mathrm{~m}, 0.6 \mathrm{H}), 7.03(\mathrm{~m}, 1 \mathrm{H}), 7.36(\mathrm{~m}, 2.5 \mathrm{H}), 7.45(\mathrm{~m}$, $2.1 \mathrm{H}), 7.53(\mathrm{~m}, 0.5 \mathrm{H}), 7.367(\mathrm{~m}, 0.3 \mathrm{H}) ;{ }^{13} \mathrm{C}$ NMR $\left(100 \mathrm{MHz}, \mathrm{CDCl}_{3}\right): \delta 16.6,21.0$, $21.2,21.8,33.6,34.5,40.7,41.7,51.8,52.0,122.9,124.0,127.5,127.7,129.4,129.6$, $129.7,130.1,132.9,133.9,135.6,135.7,136.1,136.7,141.8,143.1,144.5,144.7,152.5$, 156.8, 165.4, 165.8, 192.3, 192.6; IR (NaCl plate, $\left.\mathrm{cm}^{-1}\right)$ : 2950, 2912, 1712, 1658, 1618, $1448,1376,1279,1234,977,753,691,464$; HRMS calculated for $\mathrm{C}_{19} \mathrm{H}_{20} \mathrm{O}_{3}(\mathrm{M}+\mathrm{H})^{+}$: 297.1485, Found: 297.14871.

$\boldsymbol{E}^{3} \mathrm{~J}_{\mathrm{C}-\mathrm{H}}=6.4 \mathrm{~Hz}$ for $\mathrm{C}_{\text {ketone }}-\mathrm{H},{ }^{3} \mathrm{~J}_{\mathrm{C}-\mathrm{H}}=8.4 \mathrm{~Hz}$ for $\mathrm{C}_{\text {ester }}-\mathrm{H}$.

The following compounds were prepared by Nazarov cyclization following General Procedure II:

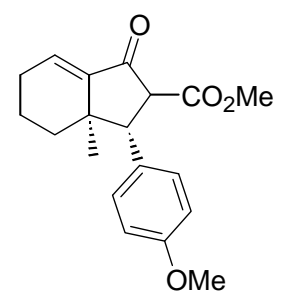


3-(4-Methoxy-phenyl)-3a-methyl-1-oxo-2,3,3a,4,5,6-hexahydro-1H-indene-2carboxylic acid methyl ester (29). Purified by flash chromatography (silica gel, ethyl acetate: hexanes $=1: 3) .{ }^{1} \mathrm{H}$ NMR $\left(400 \mathrm{MHz}, \mathrm{CDCl}_{3}\right): \delta 0.80(\mathrm{~s}, 3 \mathrm{H}), 1.51(\mathrm{~m}, 1 \mathrm{H}), 1.67$ $(\mathrm{m}, 1 \mathrm{H}), 1.85(\mathrm{~m}, 2 \mathrm{H}), 2.29(\mathrm{~m}, 1 \mathrm{H}), 2.35(\mathrm{~m}, 1 \mathrm{H}), 3.44(\mathrm{~d}, 1 \mathrm{H}, \mathrm{J}=13.1 \mathrm{~Hz}), 3.70(\mathrm{~s}$, $3 \mathrm{H}), 3.82(\mathrm{~s}, 3 \mathrm{H}), 3.92(\mathrm{~d}, 1 \mathrm{H}, \mathrm{J}=13.1 \mathrm{~Hz}), 6.84(\mathrm{t}, 1 \mathrm{H}, \mathrm{J}=3.6 \mathrm{~Hz}), 6.90(\mathrm{~d}, 2 \mathrm{H}, \mathrm{J}=8.8$ $\mathrm{Hz}), 7.18(\mathrm{~d}, 2 \mathrm{H}, \mathrm{J}=8.8 \mathrm{~Hz}) ;{ }^{13} \mathrm{C}$ NMR $\left(100 \mathrm{MHz}, \mathrm{CDCl}_{3}\right): \delta 17.9,20.3,25.4,34.5$, 40.6, 52.4, 54.9, 55.2, 56.2, 113.7, 128.2, 129.1, 135.4, 144.5, 158.7, 169.8, 197.8; IR $\left(\mathrm{NaCl}\right.$ plate, $\left.\mathrm{cm}^{-1}\right): 2933,2850,1743,1712,1647,1514,1253,1151,1033,977,842$, 736, 586; HRMS calculated for $\mathrm{C}_{19} \mathrm{H}_{22} \mathrm{O}_{4}(\mathrm{M}+\mathrm{H})^{+}: 315.1591$, Found: 315.15945 .

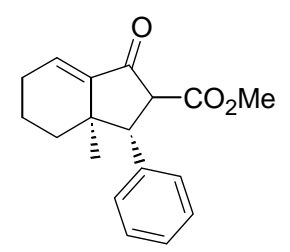

3a-Methyl-1-oxo-3-phenyl-2,3,3a,4,5,6-hexahydro-1H-indene-2-carboxylic acid methyl ester (31). Purified by flash chromatography (silica gel, ethyl acetate: hexanes = 1:3). ${ }^{1} \mathrm{H}$ NMR (400 MHz, $\left.\mathrm{CDCl}_{3}\right): \delta 0.80(\mathrm{~s}, 3 \mathrm{H}), 1.51(\mathrm{~m}, 1 \mathrm{H}), 1.68(\mathrm{~m}, 1 \mathrm{H}), 1.87(\mathrm{~m}$, $2 \mathrm{H}), 2.28(\mathrm{~m}, 1 \mathrm{H}), 2.36(\mathrm{~m}, 1 \mathrm{H}), 3.51(\mathrm{~d}, 1 \mathrm{H}, \mathrm{J}=13.1 \mathrm{~Hz}), 3.70(\mathrm{~s}, 3 \mathrm{H}), 3.99(\mathrm{~d}, 1 \mathrm{H}, \mathrm{J}=$ $13.1 \mathrm{~Hz}), 6.85(\mathrm{t}, 1 \mathrm{H}, \mathrm{J}=3.6 \mathrm{~Hz}), 7.28(\mathrm{~m}, 5 \mathrm{H}) ;{ }^{13} \mathrm{C} \mathrm{NMR}\left(100 \mathrm{MHz}, \mathrm{CDCl}_{3}\right): \delta 17.9$, 20.3, 25.3, 34.5, 40.5, 52.3, 55.4, 55.8, 127.1, 128.0, 128.2, 135.5, 136.2, 144.3, 169.6, 198.0; IR ( NaCl plate, $\left.\mathrm{cm}^{-1}\right)$ : 2940, 1744, 1714, 1648, 1435, 1333, 1277, 978, 709, 480; HRMS calculated for $\mathrm{C}_{18} \mathrm{H}_{20} \mathrm{O}_{3}(\mathrm{M}+\mathrm{Na})^{+}: 307.1305$, Found: 307.13025 .

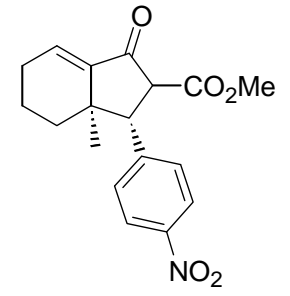

3-(4-Nitrophenyl)-3a-methyl-1-oxo-2,3,3a,4,5,6-hexahydro-1H-indene-2-carboxylic acid methyl ester (33). Purified by flash chromatography (silica gel, ethyl acetate: hexanes = 1:3). ${ }^{1} \mathrm{H}$ NMR $\left(400 \mathrm{MHz}, \mathrm{CDCl}_{3}\right): \delta 0.81(\mathrm{~s}, 3 \mathrm{H}), 1.69(\mathrm{~m}, 4 \mathrm{H}), 2.31(\mathrm{~m}, 2 \mathrm{H})$, $3.60(\mathrm{~d}, \mathrm{~J}=12.8 \mathrm{~Hz}, 1 \mathrm{H}), 3.71(\mathrm{~s}, 3 \mathrm{H}), 3.98(\mathrm{~d}, \mathrm{~J}=12.8 \mathrm{~Hz}, 1 \mathrm{H}), 6.88(\mathrm{t}, \mathrm{J}=3.6 \mathrm{~Hz}, 1 \mathrm{H})$, $7.43(\mathrm{~d}, \mathrm{~J}=8.8 \mathrm{~Hz}, 2 \mathrm{H}), 8.22(\mathrm{~d}, \mathrm{~J}=8.4 \mathrm{~Hz}, 2 \mathrm{H}) ;{ }^{13} \mathrm{C} \mathrm{NMR}\left(100 \mathrm{MHz}, \mathrm{CDCl}_{3}\right): \delta 17.9$, 20.6, 25.4, 34.6, 40.9, 52.8, 55.4, 55.8, 123.7, 129.0, 136.6, 143.5, 144.3, 147.3, 169.1, 196.6; IR ( NaCl plate, $\left.\mathrm{cm}^{-1}\right)$ : 3080, 2902, 2844, 1712, 1653, 1522, 1347, 1252, 1100 , 1038, 868, 792, 707; HRMS calculated for $\mathrm{C}_{18} \mathrm{H}_{20} \mathrm{NO}_{5}(\mathrm{M}+\mathrm{H})^{+}$: 330.1341, Found: 330.1337 .

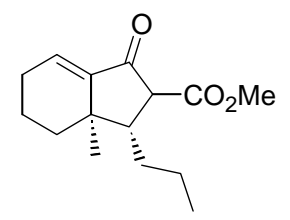

3a-Methyl-1-oxo-3-propyl-2,3,3a,4,5,6-hexahydro-1H-indene-2-carboxylic

acid methyl ester (35). Purified by flash chromatography (silica gel, ethyl acetate: hexanes = 
1:19). ${ }^{1} \mathrm{H}$ NMR (400 MHz, $\left.\mathrm{CDCl}_{3}\right): \delta 0.94(\mathrm{t}, 3 \mathrm{H}, \mathrm{J}=7.2 \mathrm{~Hz}), 0.99(\mathrm{~s}, 3 \mathrm{H}), 1.33(\mathrm{~m}, 2$ H), $1.35(\mathrm{~m}, 2 \mathrm{H}), 1.45(\mathrm{~m}, 1 \mathrm{H}), 1.79(\mathrm{~m}, 2 \mathrm{H}), 1.98(\mathrm{~m}, 1 \mathrm{H}), 2.21(\mathrm{~m}, 2 \mathrm{H}), 2.31(\mathrm{~m}, 1$ $\mathrm{H}), 3.15(\mathrm{~d}, 1 \mathrm{H}, \mathrm{J}=11.9 \mathrm{~Hz}), 3.77(\mathrm{~s}, 3 \mathrm{H}), 6.70(\mathrm{t}, 1 \mathrm{H}, \mathrm{J}=3.6 \mathrm{~Hz}) ;{ }^{13} \mathrm{C} \mathrm{NMR}(100$ $\left.\mathrm{MHz}_{\mathrm{CDCl}}\right): \delta 14.4,18.1,19.7,21.4,25.3,32.1,34.8,39.2,50.0,52.3,58.8,134.2$, 145.2, 171.1, 199.5; IR (NaCl plate, $\left.\mathrm{cm}^{-1}\right)$ : 2958, 2935, 2873, 1729, 1438, 1282, 1223 , 1199, 481; HRMS calculated for $\mathrm{C}_{15} \mathrm{H}_{22} \mathrm{O}_{3}(\mathrm{M}+\mathrm{Na})^{+}: 273.1461$, Found: 273.14633 .

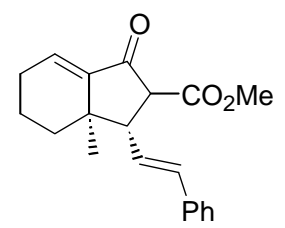

Methyl-1-oxo-3-styryl-2,3,3a,4,5,6-hexahydro-1H-indene-2-carboxylic acid methyl ester (38). Purified by flash chromatography (silica gel, ethyl acetate: hexanes $=1: 6) .{ }^{1} \mathrm{H}$ NMR (400 MHz, CDCl $)$ : $\delta 1.08(\mathrm{~s}, 3 \mathrm{H}), 1.37(\mathrm{~m}, 1 \mathrm{H}), 1.82(\mathrm{~m}, 2 \mathrm{H}), 1.97(\mathrm{~m}, 1 \mathrm{H})$, $2.36(\mathrm{~m}, 2 \mathrm{H}), 2.99\left(\mathrm{~d}-\mathrm{d}, \mathrm{J}_{1}=12.2 \mathrm{~Hz}, \mathrm{~J}_{2}=8.0 \mathrm{~Hz}, 1 \mathrm{H}\right), 3.56(\mathrm{~d}, 1 \mathrm{H}, \mathrm{J}=12.2 \mathrm{~Hz}), 3.77$ $(\mathrm{s}, 3 \mathrm{H}), 6.24\left(\mathrm{~d}-\mathrm{d}, 1 \mathrm{H}, \mathrm{J}_{1}=15.8 \mathrm{~Hz}, \mathrm{~J}_{2}=8.0 \mathrm{~Hz}\right), 6.54(\mathrm{~d}, 1 \mathrm{H}, \mathrm{J}=15.8 \mathrm{~Hz}), 6.80(\mathrm{t}, 1 \mathrm{H}$, $\mathrm{J}=3.6 \mathrm{~Hz}), 7.27(\mathrm{~d}, 1 \mathrm{H}, \mathrm{J}=6.5 \mathrm{~Hz}), 7.35(\mathrm{~d}, 2 \mathrm{H}, \mathrm{J}=6.5 \mathrm{~Hz}), 7.41(\mathrm{~d}, 2 \mathrm{H}, \mathrm{J}=7.2 \mathrm{~Hz})$;

${ }^{13} \mathrm{C}$ NMR $\left(100 \mathrm{MHz}, \mathrm{CDCl}_{3}\right): \delta 17.8,20.5,25.3,34.3,40.3,52.3,53.8,57.2,125.7$, $126.2,127.5,128.4,133.0,135.1,136.7,144.2,169.7,198.0 ;$ IR (NaCl plate, $\left.\mathrm{cm}^{-1}\right)$ : 2937, 1743, 1713, 1646, 1334, 1148, 975, 693, 449; HRMS calculated for $\mathrm{C}_{20} \mathrm{H}_{22} \mathrm{O}_{3}$ $(\mathrm{M}+\mathrm{H})^{+}: 311.1642$, Found: 311.16321 .

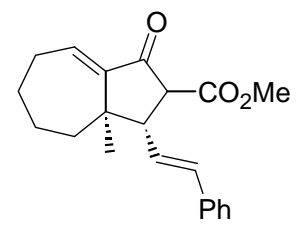

3a-Methyl-1-oxo-3-styryl-1,2,3,3a,4,5,6,7-octahydro-azulene-2-carboxylic acid methyl ester (40). Purified by flash chromatography (silica gel, ethyl acetate: hexanes = 1:6). ${ }^{1} \mathrm{H}$ NMR (400 MHz, $\left.\mathrm{CDCl}_{3}\right): \delta 1.13$ (s, $\left.3 \mathrm{H}\right), 1.47(\mathrm{~m}, 4 \mathrm{H}), 1.98(\mathrm{~m}, 2 \mathrm{H}), 2.30(\mathrm{~m}$, $1 \mathrm{H}), 2.51(\mathrm{~m}, 1 \mathrm{H}), 3.45\left(\mathrm{dd}, 1 \mathrm{H}, \mathrm{J}_{1}=8.6, \mathrm{~Hz}, \mathrm{~J}_{2}=8.6 \mathrm{~Hz}\right), 3.55(\mathrm{~d}, 1 \mathrm{H}, \mathrm{J}=16.1 \mathrm{~Hz})$, $3.75(\mathrm{~s}, 3 \mathrm{H}), 6.18\left(\mathrm{dd}, 1 \mathrm{H}, \mathrm{J}_{1}=8.6 \mathrm{~Hz}, \mathrm{~J}_{1}=8.6 \mathrm{~Hz}\right), 6.55(\mathrm{~d}, 1 \mathrm{H}, \mathrm{J}=15.7 \mathrm{~Hz}), 7.02$ (dd, $\left.1 \mathrm{H}, \mathrm{J}_{1}=3.9 \mathrm{~Hz}, \mathrm{~J}_{2}=3.9 \mathrm{~Hz}\right), 7.33(\mathrm{~m}, 5 \mathrm{H}) ;{ }^{13} \mathrm{C} \mathrm{NMR}\left(100 \mathrm{MHz}, \mathrm{CDCl}_{3}\right): \delta 18.6,24.8$, $27.3,28.3,38.4,45.9,52.3,52.8,58.0,125.4,126.2$, 127.5, 128.4, 133.9, 136.6, 139.8, 146.5, 169.7, 198.0; IR (NaCl plate, $\mathrm{cm}^{-1}$ ): 2924, 1744, 1713, 1641, 1446, 1435, 1258, 1139,695 , 446; HRMS calculated for $\mathrm{C}_{21} \mathrm{H}_{24} \mathrm{O}_{3}(\mathrm{M}+\mathrm{H})^{+}:$325.1798, Found: 325.17990 .

\section{Preparation of Compounds in Table 4}

\section{The following compounds were prepared by Knoevenagel condensation following General Procedure I:}

Knoevenagel condensation gave a mixture of isomers with a ratio of 92/8 (10E/10Z). 
<smiles>CCC/C=C(\C(C)=O)C(=O)C1=CCCCC1</smiles>

E-2-(Cyclohex-1-enecarbonyl)-hex-2-enoic acid methyl ester (42E). Purified by gravity chromatography (silica gel, ethyl acetate: hexanes $=1: 49) .{ }^{1} \mathrm{H}$ NMR $(400 \mathrm{MHz}$, $\mathrm{CDCl}_{3}$ ): $\delta 0.73$ (t, $\left.3 \mathrm{H}, \mathrm{J}=7.4 \mathrm{~Hz}\right), 1.28(\mathrm{q}, 2 \mathrm{H}, \mathrm{J}=7.4 \mathrm{~Hz}), 1.49(\mathrm{~m}, 4 \mathrm{H}), 2.08$ (dt, $2 \mathrm{H}$, $\left.\mathrm{J}_{1}=7.4 \mathrm{~Hz}, \mathrm{~J}_{2}=7.6 \mathrm{~Hz}\right), 2.10(\mathrm{~m}, 4 \mathrm{H}), 3.53(\mathrm{~s}, 3 \mathrm{H}), 6.60(\mathrm{t}, 1 \mathrm{H}, \mathrm{J}=7.6 \mathrm{~Hz}), 6.82(\mathrm{~m}$, $1 \mathrm{H}) ;{ }^{13} \mathrm{C} \mathrm{NMR}\left(100 \mathrm{MHz}, \mathrm{CDCl}_{3}\right): \delta 13.7,21.6,21.7,21.9,23.2,26.0,31.1,51.6,133.9$, 139.2, 142.9, 148.9, 164.7, 194.7; IR ( NaCl plate, $\left.\mathrm{cm}^{-1}\right): 2935,2872,1723,1659,1634$, 1435, 1280, 1226, 1213, 473; HRMS calculated for $\mathrm{C}_{14} \mathrm{H}_{20} \mathrm{O}_{3}(\mathrm{M}+\mathrm{Na})^{+}:$259.1305, Found: 259.13062.

$\boldsymbol{E}^{3} \mathrm{~J}_{\mathrm{C}-\mathrm{H}}=7.4 \mathrm{~Hz}$ for $\mathrm{C}_{\mathrm{ester}}-\mathrm{H}$.

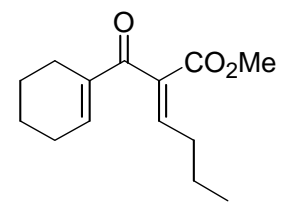

Z-2-(Cyclohex-1-enecarbonyl)-hex-2-enoic acid methyl ester (42Z). Purified by gravity chromatography (silica gel, ethyl acetate: hexanes $=1: 49) .{ }^{1} \mathrm{H}$ NMR $(400 \mathrm{MHz}$, $\left.\mathrm{CDCl}_{3}\right): \delta 0.97(\mathrm{t}, 3 \mathrm{H}, \mathrm{J}=7.4 \mathrm{~Hz}), 1.52(\mathrm{q}, 2 \mathrm{H}, \mathrm{J}=7.4 \mathrm{~Hz}), 1.66(\mathrm{~m}, 4 \mathrm{H}), 2.25(\mathrm{~m}, 4$ $\mathrm{H}), 2.53\left(\mathrm{dt}, 2 \mathrm{H}, \mathrm{J}_{1}=7.4 \mathrm{~Hz}, \mathrm{~J}_{2}=7.6 \mathrm{~Hz}\right), 3.73(\mathrm{~s}, 3 \mathrm{H}), 6.37(\mathrm{t}, 1 \mathrm{H}, \mathrm{J}=7.6 \mathrm{~Hz}), 6.70$ $(\mathrm{m}, 1 \mathrm{H}) ;{ }^{13} \mathrm{C} \mathrm{NMR}\left(100 \mathrm{MHz}, \mathrm{CDCl}_{3}\right): \delta 13.4,21.1,21.2,21.4,22.1,25.9,31.2,53.3$, 133.3, 139.6, 144.7, 146.3, 164.7, 195.2; IR ( NaCl plate, $\left.\mathrm{cm}^{-1}\right): 2935,2872,1723,1659$, $1636,1435,1280,1250,1213,473$; HRMS calculated for $\mathrm{C}_{14} \mathrm{H}_{20} \mathrm{O}_{3}(\mathrm{M}+\mathrm{Na})^{+}: 259.1305$, Found: 259.13022.

$Z^{3} \mathrm{~J}_{\mathrm{C}-\mathrm{H}}=13.4 \mathrm{~Hz}$ for $\mathrm{C}_{\text {ester }}-\mathrm{H}$.

Knoevenagel condensation gave a mixture of isomers with a ratio of 94/6 (44E/44Z).<smiles>COC(=O)C(=CC1CCCCC1)C(=O)C1=CCCCC1</smiles>

E-2-(Cyclohex-1-enecarbonyl)-3-cyclohexyl-acrylic acid methyl ester (44E). Purified by flash chromatography (silica gel, ethyl acetate: hexanes $=1: 19) .{ }^{1} \mathrm{H} \mathrm{NMR}(400 \mathrm{MHz}$, $\left.\mathrm{CDCl}_{3}\right): \delta 1.17(\mathrm{~m}, 5 \mathrm{H}), 1.63(\mathrm{~m}, 9 \mathrm{H}), 2.00(\mathrm{~m}, 1 \mathrm{H}), 2.23(\mathrm{~m}, 2 \mathrm{H}), 2.30(\mathrm{~m}, 2 \mathrm{H}), 3.69$ $(\mathrm{s}, 3 \mathrm{H}), 6.75(\mathrm{~m}, 1 \mathrm{H}), 6.77(\mathrm{~d}, 1 \mathrm{H}, \mathrm{J}=10.6 \mathrm{~Hz}) ;{ }^{13} \mathrm{C} \mathrm{NMR}\left(100 \mathrm{MHz}, \mathrm{CDCl}_{3}\right): \delta 21.5$, 21.7, 22.4, 25.0, 25.5, 26.1, 31.5, 38.7, 52.0, 131.4, 140.1, 144.8, 151.1, 165.4, 195.6; IR $\left(\mathrm{NaCl}\right.$ plate, $\left.\mathrm{cm}^{-1}\right): 2927,2852,1722,1658,1254,1228$; Elemental analysis calculated for $\mathrm{C}_{17} \mathrm{H}_{24} \mathrm{O}_{3}$ : C 73.88\%; $\mathrm{H} 8.75 \%$. Found : C 74.12\%; $\mathrm{H} 8.54 \%$. 
<smiles>CC(=O)C(=CC1CCCCC1)C(=O)C1=CCCCC1</smiles>

Z-2-(Cyclohex-1-enecarbonyl)-3-cyclohexyl-acrylic acid methyl ester (44Z). Purified by flash chromatography (silica gel, ethyl acetate: hexanes $=1: 19) .{ }^{1} \mathrm{H}$ NMR $(400 \mathrm{MHz}$, $\left.\mathrm{CDCl}_{3}\right): \delta 1.32(\mathrm{~m}, 5 \mathrm{H}), 1.65(\mathrm{~m}, 9 \mathrm{H}), 2.24(\mathrm{~m}, 4 \mathrm{H}), 2.93(\mathrm{~m}, 1 \mathrm{H}), 3.73(\mathrm{~s}, 3 \mathrm{H}), 6.16$ $(\mathrm{d}, 1 \mathrm{H}, \mathrm{J}=10.0 \mathrm{~Hz}), 6.67(\mathrm{~m}, 1 \mathrm{H}) ;{ }^{13} \mathrm{C} \mathrm{NMR}\left(100 \mathrm{MHz}, \mathrm{CDCl}_{3}\right): \delta 21.5,21.7,23.3$, $25.2,25.7,26.1,32.0,38.1,51.7,131.8,139.2,142.9,153.7,165.9,194.8 ; \mathrm{IR}(\mathrm{NaCl}$ plate, $\left.\mathrm{cm}^{-1}\right): 2928,2852,1726,1657,1212,450$; HRMS calculated for $\mathrm{C}_{17} \mathrm{H}_{24} \mathrm{O}_{3}$ $(\mathrm{M}+\mathrm{Na})^{+}: 299.1618$, Found: 299.16254 .

$Z^{3} \mathrm{~J}_{\mathrm{C}-\mathrm{H}}=6.5 \mathrm{~Hz}$ for $\mathrm{C}_{\mathrm{ketone}}-\mathrm{H},{ }^{3} \mathrm{~J}_{\mathrm{C}-\mathrm{H}}=13.4 \mathrm{~Hz}$ for $\mathrm{C}_{\mathrm{ester}}-\mathrm{H}$.

Knoevenagel condensation gave a mixture of isomers with a ratio of 75/25 (46E/46Z).<smiles>COC(=O)/C(=C/C=C/c1ccccc1)C(=O)C1=CCCCC1</smiles>

E-2-(Cyclohex-1-enecarbonyl)-5-phenyl-penta-2,4-dienoic acid methyl ester (46E). Purified by flash chromatography (silica gel, ethyl acetate: hexanes $=1: 4)$. ${ }^{1} \mathrm{H}$ NMR (400 $\left.\mathrm{MHz}, \mathrm{CDCl}_{3}\right): \delta 1.62(\mathrm{~m}, 2 \mathrm{H}), 1.68(\mathrm{~m}, 2 \mathrm{H}), 2.21(\mathrm{~m}, 2 \mathrm{H}), 2.37(\mathrm{~m}, 2 \mathrm{H}), 3.74(\mathrm{~s}, 3 \mathrm{H})$, $6.67\left(\mathrm{dd}, 2 \mathrm{H}, \mathrm{J}_{1}=11.6 \mathrm{~Hz}, \mathrm{~J}_{2}=11.7 \mathrm{~Hz}\right), 6.77(\mathrm{~m}, 1 \mathrm{H}), 6.94(\mathrm{~d}, 2 \mathrm{H}, \mathrm{J}=15.4 \mathrm{~Hz}), 7.31$ $(\mathrm{m}, 3 \mathrm{H}), 7.39(\mathrm{~d}, 2 \mathrm{H}, \mathrm{J}=6.9 \mathrm{~Hz}), 7.51(\mathrm{~d}, 1 \mathrm{H}, \mathrm{J}=11.7 \mathrm{~Hz}) ;{ }^{13} \mathrm{C}$ NMR $(100 \mathrm{MHz}$, $\left.\mathrm{CDCl}_{3}\right): \delta 21.4,21.6,22.5,26.2,52.0,123.0,127.4,128.7,129.3,131.5,135.6,140.1$, 142.2, 142.6, 145.4, 165.5, 195.3; IR ( NaCl plate, $\left.\mathrm{cm}^{-1}\right): 3024,2933,2859,1715,1654$, 1613, 1586, 1251, 1149; HRMS calculated for $\mathrm{C}_{19} \mathrm{H}_{20} \mathrm{O}_{3}(\mathrm{M}+\mathrm{H})^{+}:$297.1485, Found: 297.14816.

$\boldsymbol{E}^{3} \mathrm{~J}_{\mathrm{C}-\mathrm{H}}=7.1 \mathrm{~Hz}$ for $\mathrm{C}_{\mathrm{ketone}}-\mathrm{H},{ }^{3} \mathrm{~J}_{\mathrm{C}-\mathrm{H}}=6.8 \mathrm{~Hz}$ for $\mathrm{C}_{\mathrm{ester}}-\mathrm{H}$.

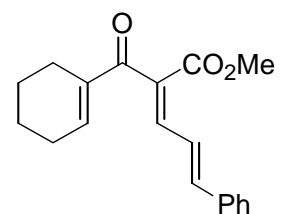

Z-2-(Cyclohex-1-enecarbonyl)-5-phenyl-penta-2,4-dienoic acid methyl ester (46Z). Purified by flash chromatography (silica gel, ethyl acetate: hexanes $=1: 4)$. ${ }^{1} \mathrm{H}$ NMR (400 $\left.\mathrm{MHz}, \mathrm{CDCl}_{3}\right): \delta 1.67(\mathrm{~m}, 4 \mathrm{H}), 2.25(\mathrm{~m}, 2 \mathrm{H}), 2.31(\mathrm{~m}, 2 \mathrm{H}), 3.79(\mathrm{~s}, 3 \mathrm{H}), 6.72(\mathrm{~m}, 1 \mathrm{H})$, $6.92\left(\mathrm{dd}, 2 \mathrm{H}, \mathrm{J}_{1}=15.4 \mathrm{~Hz}, \mathrm{~J}_{2}=15.6 \mathrm{~Hz}\right), 7.34(\mathrm{~m}, 3 \mathrm{H}), 7.53(\mathrm{~d}, 2 \mathrm{H}, \mathrm{J}=6.6 \mathrm{~Hz}), 7.80$ $\left(\mathrm{dd}, 1 \mathrm{H}, \mathrm{J}_{1}=11.4 \mathrm{~Hz}, \mathrm{~J}_{2}=15.6 \mathrm{HZ}\right) ;{ }^{13} \mathrm{C} \mathrm{NMR}\left(100 \mathrm{MHz}, \mathrm{CDCl}_{3}\right): \delta 21.4,21.7,23.3$, 26.0, 51.7, 123.8, 127.6, 128.6, 129.4, 130.6, 135.9, 139.5, 142.5, 143.7, 144.2, 165.8, 194.7; IR ( $\mathrm{NaCl}$ plate, $\left.\mathrm{cm}^{-1}\right): 3057,3032,3001,2936,2861,1711,1649,1622,1590$, 1448, 1434, 1388, 1323, 1306, 1279; HRMS calculated for $\mathrm{C}_{19} \mathrm{H}_{20} \mathrm{O}_{3}(\mathrm{M}+\mathrm{H})^{+}:$297.1485, Found: 297.14767.

$Z^{3} \mathrm{~J}_{\mathrm{C}-\mathrm{H}}=6.5 \mathrm{~Hz}$ for $\mathrm{C}_{\mathrm{ketone}}-\mathrm{H},{ }^{3} \mathrm{~J}_{\mathrm{C}-\mathrm{H}}=12.1 \mathrm{~Hz}$ for $\mathrm{C}_{\text {ester }}-\mathrm{H}$. 
The following compounds were prepared by Nazarov cyclization following General Procedure II:

Nazarov cyclization gave a mixture of products with a ratio of $1 / 2(43 \mathrm{~A} / 43 \mathrm{~B})$.

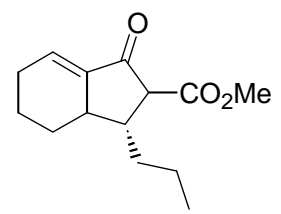

1-Oxo-3-propyl-2,3,3a,4,5,6-hexahydro-1H-indene-2-carboxylic acid methyl ester (43A). Purified by flash chromatography (silica gel, ethyl acetate: hexanes $=1: 6) .{ }^{1} \mathrm{H}$ NMR (400 MHz, $\left.\mathrm{CDCl}_{3}\right): \delta 0.94(\mathrm{t}, 3 \mathrm{H}, \mathrm{J}=7.3 \mathrm{~Hz}), 1.12(\mathrm{~m}, 1 \mathrm{H}), 1.35(\mathrm{q}, 2 \mathrm{H}, \mathrm{J}=7.7$ $\mathrm{Hz}), 1.48(\mathrm{~m}, 2 \mathrm{H}), 1.63(\mathrm{~m}, 1 \mathrm{H}), 1.91(\mathrm{~m}, 1 \mathrm{H}), 2.21(\mathrm{~m}, 4 \mathrm{H}), 2.31(\mathrm{~m}, 1 \mathrm{H}), 3.03$ (d, 1 $\mathrm{H}, \mathrm{J}=10.9 \mathrm{~Hz}), 3.78(\mathrm{~s}, 3 \mathrm{H}), 6.79(\mathrm{~m}, 1 \mathrm{H}) ;{ }^{13} \mathrm{C} \mathrm{NMR}\left(100 \mathrm{MHz}, \mathrm{CDCl}_{3}\right): \delta 14.3,20.6$, 21.6, 25.5, 27.5, 35.8, 42.1 45.6, 52.3, 61.3, 135.0, 140.0, 170.8, 198.8; IR (NaCl plate, $\left.\mathrm{cm}^{-1}\right): 2951,2933,1738,1699,1651,1436,1255,1154,912,731,483$; HRMS calculated for $\mathrm{C}_{14} \mathrm{H}_{20} \mathrm{O}_{3}(\mathrm{M}+\mathrm{Na})^{+}:$259.1305, Found: 259.13025 .

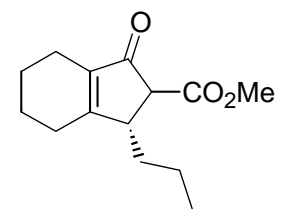

1-Oxo-3-propyl-2,3,4,5,6,7-hexahydro-1H-indene-2-carboxylic acid methyl ester (43B). Purified by flash chromatography (silica gel, ethyl acetate: hexanes $=1: 3$ ). ${ }^{1} \mathrm{H}$ NMR (400 MHz, $\left.\mathrm{CDCl}_{3}\right): \delta 0.93(\mathrm{t}, 3 \mathrm{H}, \mathrm{J}=6.9 \mathrm{~Hz}), 1.31(\mathrm{~m}, 4 \mathrm{H}), 1.73(\mathrm{~m}, 4 \mathrm{H}), 2.13$ $(\mathrm{m}, 2 \mathrm{H}), 2.29(\mathrm{~m}, 2 \mathrm{H}), 3.08(\mathrm{~m}, 2 \mathrm{H}), 3.76(\mathrm{~s}, 3 \mathrm{H}) ;{ }^{13} \mathrm{C}$ NMR $\left(100 \mathrm{MHz}, \mathrm{CDCl}_{3}\right)$ : $\delta 13.9,20.0,20.2,21.3,21.9,26.3,34.1,46.3,52.4,57.8,136.7,170.2,176.4,198.8$; IR $\left(\mathrm{NaCl}\right.$ plate, $\left.\mathrm{cm}^{-1}\right): 2951,2933,1738,1699,1644,1436,1277,1253,912,731,485$; HRMS calculated for $\mathrm{C}_{14} \mathrm{H}_{20} \mathrm{O}_{3}(\mathrm{M}+\mathrm{Na})^{+}: 259.1305$, Found: 259.13034 .

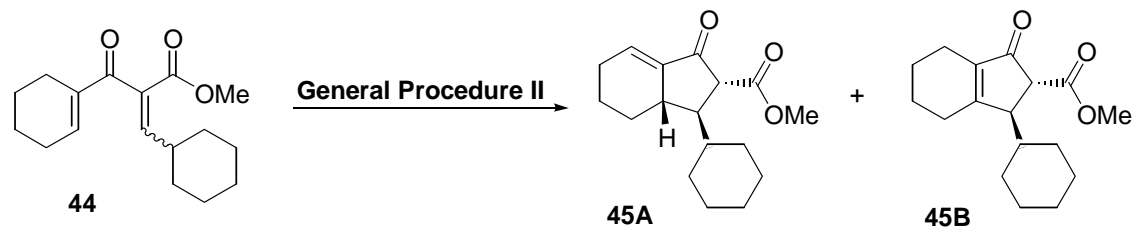

45A and 45B was obtained on Nazarov cyclization on 44 following General Procedure II. Purified by flash chromatography (silica gel, ethyl acetate: hexanes =1:3)

Compound 45A was obtained in 53\% yield. ${ }^{1} \mathbf{H}$ NMR (400 $\left.\mathbf{M H z}, \mathbf{C D C l}_{3}\right) \delta 6.79$ (t, $J$ $=3.6 \mathrm{~Hz}, 1 \mathrm{H}), 3.76(\mathrm{~s}, 3 \mathrm{H}), 3.16(\mathrm{~d}, J=11.6,1 \mathrm{H}), 2.36(\mathrm{~m}, 2 \mathrm{H}), 2.20(\mathrm{~m}, 2 \mathrm{H}), 2.12(\mathrm{~m}$, 1H), $1.76(\mathrm{~m}, 1 \mathrm{H}), 1.65(\mathrm{~m}, 5 \mathrm{H}), 1.55(\mathrm{~m}, 2 \mathrm{H}), 1.15(\mathrm{~m}, 6 \mathrm{H}) ;{ }^{13} \mathbf{C} \mathbf{N M R}\left(\mathbf{C D C l}_{3}, \mathbf{1 0 0}\right.$ MHz) $\delta 199.2,171.2,140.2,135.4,57.8,52.4,51.1,39.7,38.7,31.7,29.3,28.4,26.7$, 26.5, 26.3, 25.6, 21.7; IR (NaCl plate, $\mathbf{~ c m}^{-1}$ ): 2925, 2853, 1744, 1713, 1651, 1447, 1336, 1259, 1149, 979, 422; Elemental analysis calculated for $\mathrm{C}_{17} \mathrm{H}_{24} \mathrm{O}_{3}$ : C 73.88\%; $\mathrm{H} 8.75 \%$. Found : C $73.98 \%$; H 8.69\%.

Compound 45B was obtained in 17\% yield. ${ }^{1} \mathbf{H}$ NMR (400 $\left.\mathbf{~ M H z , ~} \mathbf{C D C l}_{3}\right) \delta 3.75$ (s, 3H), $3.22(\mathrm{~d}, J=2.4 \mathrm{~Hz}, 1 \mathrm{H}), 3.11$ (br. s, $1 \mathrm{H}), 2.29(\mathrm{~m}, 2 \mathrm{H}), 2.12(\mathrm{~m}, 2 \mathrm{H}), 1.71(\mathrm{~m}$, 
9H), $1.26(\mathrm{~m}, 5 \mathrm{H}), 0.83(\mathrm{~m}, 1 \mathrm{H}) ;{ }^{13} \mathbf{C} \mathbf{~ N M R}\left(\mathbf{C D C l}_{3}, \mathbf{1 0 0} \mathbf{~ M H z}\right) \delta 200.9,175.6,170.5$, 137.6, 53.3, 52.3, 51.8, 37.9, 31.5, 26.7, 26.4, 26.2, 26.1, 26.0, 22.0, 21.4, 20.1; IR (NaCl plate, $\mathbf{~ c m}^{-1}$ ) 2925, 2853, 1738, 1702, 1644, 1439, 1263, 1155, 897, 730; Elemental analysis calculated for $\mathrm{C}_{17} \mathrm{H}_{24} \mathrm{O}_{3}$ : C 73.88\%; H 8.75\%. Found : $\mathrm{C} 74.07 \%$; $\mathrm{H} 8.60 \%$.

Nazarov cyclization gave a mixture of products with a ratio of $1 / 1.7(47 \mathrm{~A} / 47 \mathrm{~B})$.

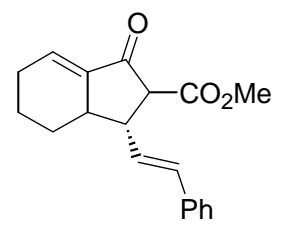

1-Oxo-3-styryl-2,3,3a,4,5,6-hexahydro-1H-indene-2-carboxylic acid methyl ester (47A). Purified by flash chromatography (silica gel, ethyl acetate: hexanes $=1: 6$ ). ${ }^{1} \mathrm{H}$ NMR (400 MHz, CDCl $)$ : $\delta 1.51(\mathrm{~m}, 1 \mathrm{H}), 1.74(\mathrm{~m}, 1 \mathrm{H}), 2.22(\mathrm{~m}, 2 \mathrm{H}), 2.34(\mathrm{~m}, 1 \mathrm{H})$, $2.41(\mathrm{~m}, 1 \mathrm{H}), 2.86(\mathrm{~m}, 1 \mathrm{H}), 3.30(\mathrm{~d}, 1 \mathrm{H}, \mathrm{J}=11.9 \mathrm{~Hz}), 3.71(\mathrm{~d}, 1 \mathrm{H}, \mathrm{J}=12.7 \mathrm{~Hz}), 3.75$ $(\mathrm{s}, 3 \mathrm{H}), 6.15\left(\mathrm{dd}, 1 \mathrm{H}, \mathrm{J}_{1}=8.2 \mathrm{~Hz}, \mathrm{~J}_{2}=8.2 \mathrm{~Hz}\right), 6.20(\mathrm{~d}, 1 \mathrm{H}, \mathrm{J}=15.8 \mathrm{~Hz}), 6.85(\mathrm{~m}, 1 \mathrm{H})$, 7.33 (m, $5 \mathrm{H}) ;{ }^{13} \mathrm{C}$ NMR $\left(100 \mathrm{MHz}, \mathrm{CDCl}_{3}\right): \delta 21.4,25.5,26.7,41.8,49.4,52.2,60.8$, 126.2, 127.5, 128.4, 128.5, 132.2, 135.7, 136.6, 138.8, 169.3, 197.3; IR (NaCl plate, $\mathrm{cm}^{-}$ $\left.{ }^{1}\right): 3080,3057,3026,3000,2935,2860,1741,1713,1650,1545,1448,1435,1370,1332$, 1261, 1214, 1152, 972; HRMS calculated for $\mathrm{C}_{19} \mathrm{H}_{20} \mathrm{O}_{3}(\mathrm{M}+\mathrm{H})^{+}$: 297.1485, Found: 297.14844 .

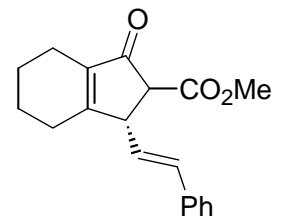

1-Oxo-3-styryl-2,3,4,5,6,7-hexahydro-1H-indene-2-carboxylic acid methyl ester (47B). Purified by flash chromatography (silica gel, ethyl acetate: hexanes $=1: 3$ ). ${ }^{1} \mathrm{H}$ NMR (400 MHz, CDCl $)$ ): $\delta 1.68(\mathrm{~m}, 4 \mathrm{H}), 2.18(\mathrm{~m}, 2 \mathrm{H}), 2.32(\mathrm{~m}, 2 \mathrm{H}), 3.30(\mathrm{~m}, 1 \mathrm{H})$, $3.77(\mathrm{~s}, 3 \mathrm{H}), 3.87(\mathrm{~d}, 1 \mathrm{H}, \mathrm{J}=8.0 \mathrm{~Hz}), 5.93\left(\mathrm{dd}, 1 \mathrm{H}, \mathrm{J}_{1}=15.7 \mathrm{~Hz}, \mathrm{~J}_{2}=8.0 \mathrm{~Hz}\right), 6.59(\mathrm{~d}$, $1 \mathrm{H}, \mathrm{J}=15.7 \mathrm{~Hz}), 7.30(\mathrm{~m}, 5 \mathrm{H}) ;{ }^{13} \mathrm{C}$ NMR $\left(100 \mathrm{MHz}, \mathrm{CDCl}_{3}\right): \delta 20.2,21.3,21.9,26.5$, 50.0, 52.5, 58.5, 126.2, 127.7, 127.8, 128.5, 133.5, 136.2, 137.1, 169.3, 174.5, 199.8; IR $\left(\mathrm{NaCl}\right.$ plate, $\left.\mathrm{cm}^{-1}\right): 3080,3057,3026,3001,2936,2861,1738,1704,1644,1600,1445$, $1434,1418,1388,1327,1238,1192,1158,971$; HRMS calculated for $\mathrm{C}_{19} \mathrm{H}_{20} \mathrm{O}_{3}$ $(\mathrm{M}+\mathrm{Na})^{+}: 319.1305$, Found: 319.13062.

\section{Procedure of Ozonolysis of 38}

$\mathrm{O}_{3}$ was bubbled through a solution of $\mathbf{3 8}(92 \mathrm{mg}, 0.3 \mathrm{mmol})$ in dichloromethane $(3.0 \mathrm{ml})$ at $-78{ }^{\circ} \mathrm{C}$ for 20 seconds. After stirring the reaction for 2 minutes, $\mathrm{O}_{2}$ was bubbled through the solution. $\mathrm{Me}_{2} \mathrm{~S}(0.22 \mathrm{ml}, 3.0 \mathrm{mmol})$ was then added at $-78{ }^{\circ} \mathrm{C}$ and the reaction was allowed to warm to room temperature overnight. The volatile compounds were removed under reduced pressure. The solid residue was subjected to flash chromatography (silica gel, ethyl acetate: hexanes $=1: 2$ ) to give the desired aldehyde 48 as a yellow oil (43 $\mathrm{mg}, 61 \%)$. 


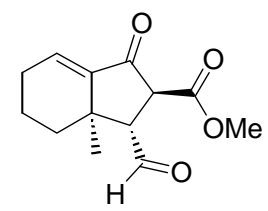

3-Formyl-3a-methyl-1-oxo-2,3,3a,4,5,6-hexahydro-1H-indene-2-carboxylic acid methyl ester (48). ${ }^{1} \mathrm{H}$ NMR (400 MHz, $\left.\mathrm{CDCl}_{3}\right): \delta 1.04$ (s, $\left.3 \mathrm{H}\right), 1.73(\mathrm{~m}, 4 \mathrm{H}), 2.28$ (m, 2 H), 3.39 (d, 1 H, J = 11.5 Hz), $3.77(\mathrm{~s}, 3 \mathrm{H}), 3.86(\mathrm{~d}, 1 \mathrm{H}, \mathrm{J}=11.5 \mathrm{~Hz}), 6.76$ (t, $1 \mathrm{H}, \mathrm{J}=$ $3.6 \mathrm{~Hz}), 9.90(\mathrm{~s}, 1 \mathrm{H}) ;{ }^{13} \mathrm{C} \mathrm{NMR}\left(100 \mathrm{MHz}, \mathrm{CDCl}_{3}\right): \delta 17.7,21.5,25.0,35.2,39.0,51.4$, 52.6, 61.2, 135.6, 143.3, 168.5, 195.6, 199.2; IR (NaCl plate, $\left.\mathrm{cm}^{-1}\right)$ : 2950, 2824, 1742, 1715, 1648, 1593, 1453, 1295, 1202, 1026, 481; HRMS calculated for $\mathrm{C}_{13} \mathrm{H}_{16} \mathrm{O}_{4}(\mathrm{M}+\mathrm{Na})^{+}:$259.0941, Found: 259.09497.

\section{Preparation of Compounds in Table 6}

All compounds have been characterized and previously reported. ${ }^{3}$

\section{Preparation of Alkynes S7-S11}<smiles>CC(=O)c1cc(O)cc(O)c1</smiles><smiles>CC(=O)c1cc(O[AsH2])cc(O[AsH2])c1</smiles>

S5

Acetophenone S5: In a dry $250 \mathrm{~mL}$ round-bottom flask was weighed 3',5'acetophenone (5.00 g, $0.03 \mathrm{~mol})$, imidazole (7.88 g, $0.12 \mathrm{~mol})$, and $\mathrm{CH}_{2} \mathrm{Cl}_{2}(80 \mathrm{~mL})$, and the solution was stirred while TIPS-Cl $(24.0 \mathrm{~mL}, 0.11 \mathrm{~mol})$ was added. Stirring continued until TLC showed full conversion to diprotected material, at about $6 \mathrm{~h}$. The solution was quenched with water $(100 \mathrm{~mL})$, the layers were separated, and the aqueous layer was extracted with $\mathrm{CH}_{2} \mathrm{Cl}_{2}(3 \times 50 \mathrm{~mL})$. The organic phases were combined and dried over $\mathrm{MgSO}_{4}$, filtered, and distilled under vacuum $\left(1 \mathrm{~mm} \mathrm{Hg}, 180^{\circ} \mathrm{C}\right)$ to yield 13.08 $\mathrm{g}(85 \%)$ of S5.

${ }^{1} \mathbf{H}$ NMR (CDCl, 400 MHz): $\delta 1.10(\mathrm{~d}, \mathrm{~J}=9.0 \mathrm{~Hz}, 36 \mathrm{H}), 1.26(\mathrm{~m}, 6 \mathrm{H}), 2.53(\mathrm{~s}, 3 \mathrm{H})$, $\left.6.62(\mathrm{t}, \mathrm{J}=2.4 \mathrm{~Hz}, 1 \mathrm{H}), 7.06(\mathrm{~d}, \mathrm{~J}=2.4 \mathrm{~Hz}, 2 \mathrm{H}) ;{ }^{13} \mathbf{C} \mathbf{N M R}_{(\mathbf{C D C l}}, \mathbf{1 0 0} \mathbf{M H z}\right): \delta 12.6$, 17.9, 26.8, 112.9, 116.4, 138.9, 157.12, 197.7; IR (neat, $\mathbf{~ c m}^{-1}$ ): 2945.6, 2867.8, 1690.2, 1439.9, 1357.5, 1228.0. 


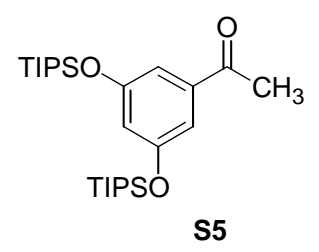

i) LDA, THF, $-70^{\circ} \mathrm{C}$

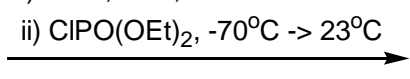

iii) LDA, THF, $-70^{\circ} \mathrm{C}->23^{\circ} \mathrm{C}$

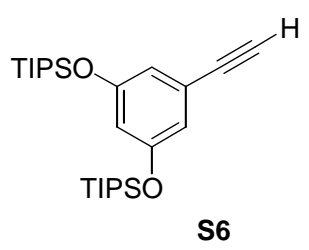

Terminal Alkyne S6: In a dry $100 \mathrm{~mL}$ round-bottom flask was measured THF (15 mL), diisopropylamine $(2.1 \mathrm{~mL}, 0.015 \mathrm{~mol})$, the solution was cooled to $0^{\circ} \mathrm{C}$ and $n$-butyllithium (1.4 $\mathrm{M}$ in hexanes, $10.9 \mathrm{~mL} 0.015 \mathrm{~mol}$ ) was added. The solution was stirred for $30 \mathrm{~min}$, cooled to $-70^{\circ} \mathrm{C}$, and a solution of $\mathbf{S 5}(5.89 \mathrm{~g}, 0.013 \mathrm{~mol})$ in THF $(15 \mathrm{~mL})$ was added and stirred for $2 \mathrm{~h}$ at $-70^{\circ} \mathrm{C}$. Diethylchlorophosphate $(2.2 \mathrm{~mL}, 0.015 \mathrm{~mol})$ was added dropwise at $-70^{\circ} \mathrm{C}$, the solution was stirred for $20 \mathrm{~min}$, then the cold bath was removed and stirring continued for $5.5 \mathrm{~h}$. A solution of LDA was prepared in a dry $250 \mathrm{~mL}$ roundbottom flask using diisopropylamine $(4.7 \mathrm{~mL}, 0.033 \mathrm{~mol})$, THF $(30 \mathrm{~mL})$, and $n$ butyllithium (1.4 M in hexanes, $23.8 \mathrm{~mL}, 0.033 \mathrm{~mol}$ ) at $0^{\circ} \mathrm{C}$ and stirred for $30 \mathrm{~min}$, after which the reaction mixture was added via cannula, and stirred for another $16 \mathrm{~h}$, allowing the cold bath to warm to $23^{\circ} \mathrm{C}$. The solution was quenched with water $(100 \mathrm{~mL})$, diluted with hexanes $(100 \mathrm{~mL})$, and the layers were separated. The aqueous phase was extracted with hexanes $(2 \times 100 \mathrm{~mL})$, the organic layers were combined and washed with $\mathrm{HCl}(2 \mathrm{M}$, $2 \times 50 \mathrm{~mL}$ ), water $(2 \times 100 \mathrm{~mL}), \mathrm{NaHCO}_{3}$ (saturated aqueous solution, $100 \mathrm{~mL}$ ), and brine $(100 \mathrm{~mL})$. The organic layer was dried over $\mathrm{MgSO}_{4}$, filtered, and concentrated. The crude material was vacuum distilled $\left(0.05 \mathrm{~mm} \mathrm{Hg}, 160^{\circ} \mathrm{C}\right)$, yielding $3.78 \mathrm{~g}(67 \%)$ of S6.

${ }^{1}$ H NMR (CDCl 3 , 400 MHz): $\delta 1.12(\mathrm{~d}, \mathrm{~J}=9.0 \mathrm{~Hz}, 36 \mathrm{H}), 1.24(\mathrm{~m}, 6 \mathrm{H}), 3.00(\mathrm{~s}, 1 \mathrm{H})$, $6.44(\mathrm{t}, \mathrm{J}=2.0 \mathrm{~Hz}, 1 \mathrm{H}), 6.62(\mathrm{~d}, \mathrm{~J}=2.0 \mathrm{~Hz}, 2 \mathrm{H}) ;{ }^{13} \mathbf{C} \mathbf{N M R}\left(\mathbf{C D C l}_{3}, \mathbf{1 0 0} \mathbf{M H z}\right): \delta 12.6$, 17.8, 83.7 113.3, 116.8, 122.9, 156.7; IR (neat, $\mathbf{~ c m}^{-1}$ ): 3313.7, 3073.1, 2944.9, 2892.0, 2867.4, 1581.4, 1428.0, 1345.6; HRMS (EI) calculated for $\mathrm{C}_{26} \mathrm{H}_{46} \mathrm{O}_{2} \mathrm{Si}_{2}$ 446.3031, found 446.3030 .
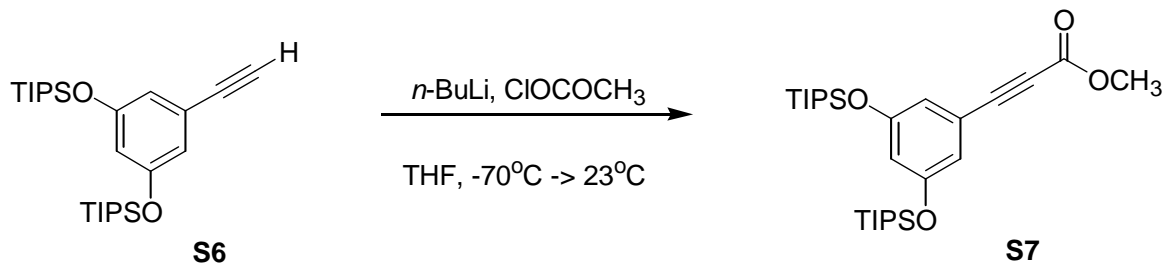

Alkynyl Ester S7: In a dry $100 \mathrm{~mL}$ round-bottom flask was weighed S6 (3.78 g, 8.5 $\mathrm{mmol})$ and THF $(40 \mathrm{~mL})$, and the solution was cooled to $-70^{\circ} \mathrm{C}$, then $n$-butyllithium $(1.4$ $\mathrm{M}$ in hexanes, $7.5 \mathrm{~mL}, 10.4 \mathrm{mmol}$ ) was added and stirred for $1 \mathrm{~h}$. Methyl chloroformate (1.2 $\mathrm{mL}, 15.5 \mathrm{mmol})$ was added dropwise and stirring continued for $16 \mathrm{~h}$, allowing the cold bath to warm to $23^{\circ} \mathrm{C}$. The solution was quenched with water $(50 \mathrm{~mL})$, the layers were separated and the aqueous phase was extracted with hexanes $(2 \times 50 \mathrm{~mL})$. The organic layers were combined and washed with water $(2 \times 100 \mathrm{~mL})$, then brine $(100 \mathrm{~mL})$. The organic layer was dried over $\mathrm{MgSO}_{4}$, filtered, and concentrated. The crude material was purified by column chromatography (32:1 hexanes:ethyl acetate), yielding $4.2 \mathrm{~g}$ $(98 \%)$ of S7. 
${ }^{1} \mathbf{H}$ NMR (CDCl 3 , $\left.\mathbf{4 0 0} \mathbf{~ M H z}\right): \delta 1.10(\mathrm{~d}, \mathrm{~J}=9.0 \mathrm{~Hz}, 36 \mathrm{H}), 1.24(\mathrm{~m}, 6 \mathrm{H}), 3.83(\mathrm{~s}, 3 \mathrm{H})$, $\left.6.52(\mathrm{t}, \mathrm{J}=2.4 \mathrm{~Hz}, 1 \mathrm{H}), 6.70(\mathrm{~d}, \mathrm{~J}=2.4 \mathrm{~Hz}, 2 \mathrm{H}) ;{ }^{13} \mathbf{C ~ N M R}_{(\mathbf{C D C l}}, \mathbf{1 0 0} \mathbf{M H z}\right): \delta 12.6$, 17.4, 52.7, 79.6, 86.6, 115.3, 117.5, 120.3, 154.5, 157.0; IR (neat, $\mathbf{~ c m}^{-1}$ ): 2943.7, 2891.1, 2866.5, 2218.9, 1715.6, 1577.7, 1462.4, 1427.2, 1241.6.

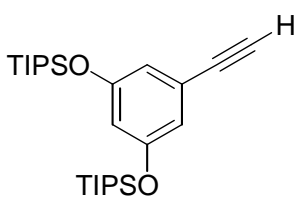

S6

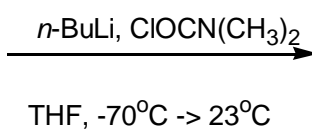

THF, $-70^{\circ} \mathrm{C}->23^{\circ} \mathrm{C}$

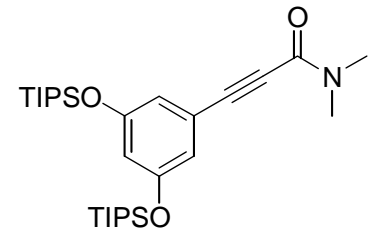

S8

Alkynyl Amide S8: In a dry $50 \mathrm{~mL}$ round-bottom flask was weighed S6 (2.68 g, 6.0 $\mathrm{mmol})$ and THF $(30 \mathrm{~mL})$, and the solution was cooled to $-70^{\circ} \mathrm{C}$, then $n$-butyllithium $(1.5$ $\mathrm{M}$ in hexanes, $4.8 \mathrm{~mL}, 7.2 \mathrm{mmol}$ ) was added and stirred for $6 \mathrm{~h}$. Dimethyl carbamyl chloride $(0.9 \mathrm{~mL}, 9.2 \mathrm{mmol})$ was added dropwise and stirring continued for $16 \mathrm{~h}$, allowing the cold bath to warm to $23^{\circ} \mathrm{C}$. The solution was quenched with water $(50 \mathrm{~mL})$, the layers were separated and the aqueous phase was extracted with ethyl acetate $(2 \times 50$ $\mathrm{mL})$. The organic layers were combined and washed with water $(2 \times 100 \mathrm{~mL})$, then brine $(100 \mathrm{~mL})$. The organic layer was dried over $\mathrm{MgSO}_{4}$, filtered, and concentrated. The crude material was purified by column chromatography (4:1 hexanes:ethyl acetate), yielding $2.4 \mathrm{~g}(77 \%)$ of $\mathbf{S 8}$.

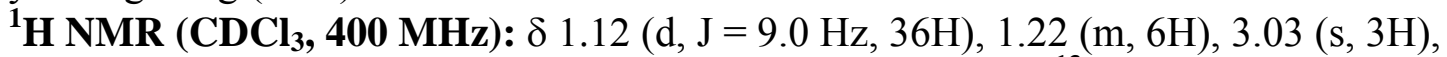
$3.28(\mathrm{~s}, 3 \mathrm{H}), 6.49(\mathrm{t}, \mathrm{J}=2.4 \mathrm{~Hz}, 1 \mathrm{H}), 6.66(\mathrm{~d}, \mathrm{~J}=2.4 \mathrm{~Hz}, 2 \mathrm{H}) ;{ }^{13} \mathbf{C} \mathbf{~ N M R}\left(\mathbf{C D C l}_{3}, \mathbf{1 0 0}\right.$ MHz): $\delta 12.6,17.8,34.2,38.4,80.8,90.3,114.4,116.9,121.4,154.6,156.9$; IR (neat, $\left.\mathbf{c m}^{-1}\right): 2943.2,2866.0,1577.7,1427.2,1350.1,1170.7,1028.0$.

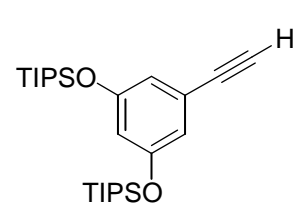

S6

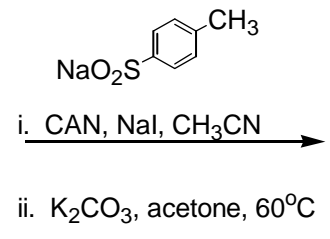

ii. $\mathrm{K}_{2} \mathrm{CO}_{3}$, acetone, $60^{\circ} \mathrm{C}$

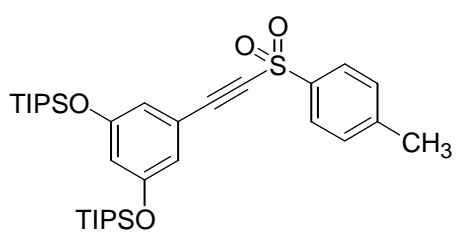

s9

Alkynyl Sulfone S9: ${ }^{4}$ In a dry $50 \mathrm{~mL}$ round-bottom flask, sodium p-toluenesulfinate (325 mg, $1.8 \mathrm{mmol}$ ) was combined with sodium iodide (445 mg, $3.0 \mathrm{mmol}$ ), S6 (725 mg, $1.6 \mathrm{mmol})$, and acetonitrile $(6 \mathrm{~mL})$, which formed a chunky suspension upon stirring. Ceric ammonium nitrate $(2.1 \mathrm{~g}, 3.8 \mathrm{mmol})$ was dissolved in acetonitrile $(9 \mathrm{~mL})$ and added to the above solution, which then turned dark brown, and was stirred for $5 \mathrm{~h}$. Acetonitrile was removed under vacuum, then potassium carbonate $(571 \mathrm{mg}, 4.0 \mathrm{mmol})$ was added, along with acetone $(8 \mathrm{~mL})$. This mixture was heated with stirring at $60^{\circ} \mathrm{C}$ for $12 \mathrm{~h}$. The reaction was quenched with water, the layers were separated, and the organic phase was washed with ammonium chloride $(25 \mathrm{~mL})$, then brine $(25 \mathrm{~mL})$. The organic phase was dried over $\mathrm{MgSO}_{4}$, filtered, and concentrated. The crude material was purified by column chromatography (16:1 hexanes:ethyl acetate), yielding $800 \mathrm{mg}(83 \%)$ of $\mathbf{S 9}$. 
${ }^{1} \mathbf{H}$ NMR (CDCl 3 , $\left.\mathbf{4 0 0} \mathbf{~ M H z}\right): \delta 1.10(\mathrm{~d}, \mathrm{~J}=9.0 \mathrm{~Hz}, 36 \mathrm{H}), 1.23(\mathrm{~m}, 6 \mathrm{H}), 2.39(\mathrm{~s}, 3 \mathrm{H})$, $6.30(\mathrm{~d}, \mathrm{~J}=2.0 \mathrm{~Hz}, 2 \mathrm{H}), 6.33(\mathrm{t}, \mathrm{J}=2.0 \mathrm{~Hz}, 1 \mathrm{H}), 7.20(\mathrm{~d}, \mathrm{~J}=8.0 \mathrm{~Hz}, 2 \mathrm{H}), 7.54(\mathrm{~d}, \mathrm{~J}=6.8$ $\mathrm{Hz}, 2 \mathrm{H}) ;{ }^{13} \mathbf{C}$ NMR (CDCl 3 , $\left.100 \mathbf{M H z}\right): \delta 12.6,17.9,21.6,112.3,112.9,113.8,128.0$, 129.6, 137.5, 140.8, 141.0, 144.4, 156.2; IR (neat, $\mathbf{~ c m}^{-1}$ ): 2943.7, 2925.8, 2866.0, $1574.3,1430.1,1325.5,1149.9,1029.9$.

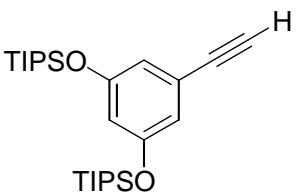

S6

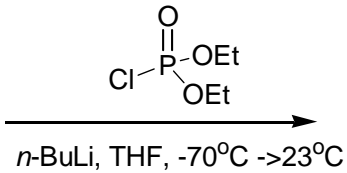

$n$-BuLi, THF, $-70^{\circ} \mathrm{C}->23^{\circ} \mathrm{C}$

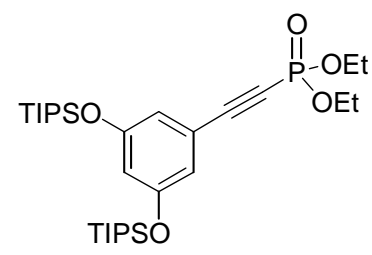

S10

Alkynyl Phosphate S10: In a dry $50 \mathrm{~mL}$ round-bottom flask was weighed S6 (1.1 g, 2.5 $\mathrm{mmol})$ and THF $(10 \mathrm{~mL})$, and the solution was cooled to $-70^{\circ} \mathrm{C}$, then $n$-butyllithium $(1.6$ $\mathrm{M}$ in hexanes, $3.1 \mathrm{~mL}, 5.0 \mathrm{mmol}$ ) was added and stirred for $30 \mathrm{~min}$. Diethyl chlorophosphate $(0.8 \mathrm{~mL}, 5.2 \mathrm{mmol})$ was added dropwise and stirring continued for $16 \mathrm{~h}$, allowing the cold bath to warm to $23^{\circ} \mathrm{C}$. The solution was quenched with water $(20 \mathrm{~mL})$, the layers were separated and the aqueous phase was extracted with diethyl ether $(2 \times 20$ $\mathrm{mL})$. The organic layers were combined and washed with water $(2 \times 25 \mathrm{~mL})$, then brine $(40 \mathrm{~mL})$. The organic layer was dried over $\mathrm{MgSO}_{4}$, filtered, and concentrated. The crude material was purified by column chromatography (4:1 hexanes:ethyl acetate), yielding $400 \mathrm{mg}(27 \%)$ of $\mathbf{S 1 0}$.

${ }^{1} \mathbf{H}$ NMR (CDCl 3 , 400 MHz): $\delta 1.10(\mathrm{~d}, \mathrm{~J}=9.0 \mathrm{~Hz}, 36 \mathrm{H}), 1.25(\mathrm{~m}, 12 \mathrm{H}), 1.40(\mathrm{t}, \mathrm{J}=7.2$ $\mathrm{Hz}, 6 \mathrm{H}), 4.23(\mathrm{~m}, 4 \mathrm{H}), 6.51(\mathrm{t}, \mathrm{J}=2.0 \mathrm{~Hz}, 1 \mathrm{H}), 6.67(\mathrm{~d}, \mathrm{~J}=2.0 \mathrm{~Hz}, 2 \mathrm{H}) ;{ }^{13} \mathbf{C} \mathbf{N M R}$ $\left(\mathbf{C D C l}_{3}, \mathbf{1 0 0} \mathbf{M H z}\right): \delta 12.6,16.1,17.8,63.2,76.1,79.1,98.9,99.4,110.1,115.2,117.1$, 120.4, 157.0; IR (neat, $\mathbf{c m}^{-1}$ ): 2942.2, 2865.5, 2185.7, 1581.5, 1462.9, 1428.7, 1165.4, 1007.7.

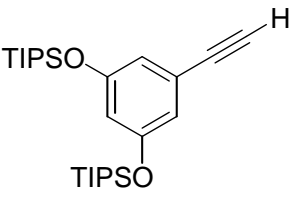

S6

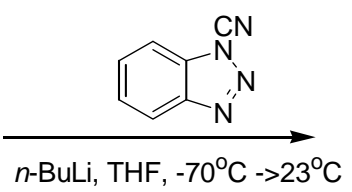

$n$-BuLi, THF, $-70^{\circ} \mathrm{C}->23^{\circ} \mathrm{C}$

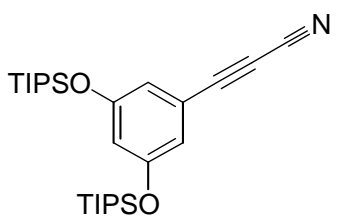

S11

Alkynyl Nitrile S11: ${ }^{5}$ In a dry $25 \mathrm{~mL}$ round-bottom flask was measured 1cyanobenzotriazole $(264 \mathrm{mg}, 1.8 \mathrm{mmol})$ and THF $(2.6 \mathrm{~mL})$, and the solution was cooled to $-70^{\circ} \mathrm{C}$, then a pre-stirred and -cooled solution of $n$-butyllithium $(1.5 \mathrm{M}$ in hexanes, 1.0 $\mathrm{mL}, 1.5 \mathrm{mmol})$, $\mathbf{S 6}(600 \mathrm{mg}, 1.3 \mathrm{mmol})$ and THF $(5.5 \mathrm{~mL})$ was added via cannula and stirred for $20 \mathrm{~h}$, allowing the cold bath to warm to $23^{\circ} \mathrm{C}$. The solution was quenched with water $(20 \mathrm{~mL})$, the layers were separated and the aqueous phase was extracted with diethyl ether $(2 \times 20 \mathrm{~mL})$. The organic layers were combined and washed with water $(2 \times$ $25 \mathrm{~mL})$, then brine $(40 \mathrm{~mL})$. The organic layer was dried over $\mathrm{MgSO}_{4}$, filtered, and concentrated. The crude material was purified by column chromatography (16:1 hexanes:ethyl acetate), yielding $454 \mathrm{mg}$ (74\%) of S11. 
${ }^{1} \mathbf{H}$ NMR (CDCl 3 , 400 MHz): $\delta 1.10(\mathrm{~d}, \mathrm{~J}=9.0 \mathrm{~Hz}, 36 \mathrm{H}), 1.24(\mathrm{~m}, 6 \mathrm{H}), 6.58(\mathrm{t}, \mathrm{J}=2.0$ $\mathrm{Hz}, 1 \mathrm{H}), 6.71(\mathrm{~d}, \mathrm{~J}=2.0 \mathrm{~Hz}, 2 \mathrm{H}) ;{ }^{13} \mathbf{C} \mathbf{~ N M R}\left(\mathbf{C D C l}_{3}, \mathbf{1 0 0} \mathbf{M H z}\right): \delta 12.5,17.7,62.4,82.8$, 105.3, 113.2, 116.4, 116.8, 117.7, 118.3, 157.2; IR (neat, $\mathbf{~ c m}^{-1}$ ): 3013.5, 2948.5, 2760.4, $2727.7,1937.2,1585.1,1429.9,1052.0$.

\section{Preparation of Compounds in Table 7}

The following compounds were prepared following reaction conditions modified in our group based on methodology developed by Padwa. ${ }^{6}$ Our one-pot procedure commences with a sequential $3+2$ cyclization of an alkyne and a nitrone to form an isoxazoline (II), followed by oxidative extrusion of nitrosomethane by $m$-CPBA to form the appropriate divinyl ketone (III) according to the following general equation:

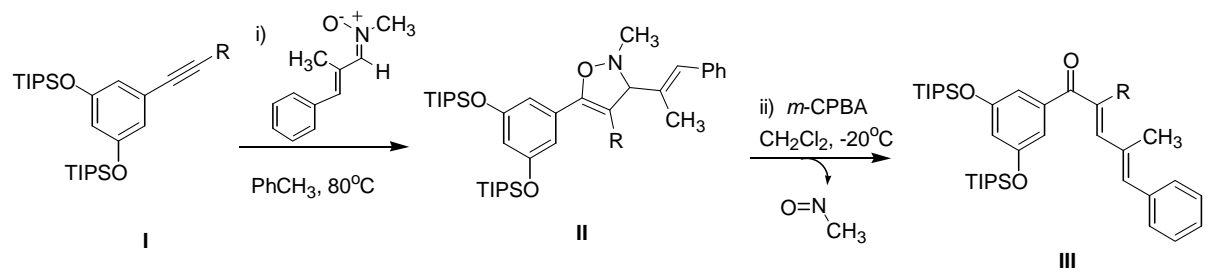

This two-step procedure was applied to the following compounds:

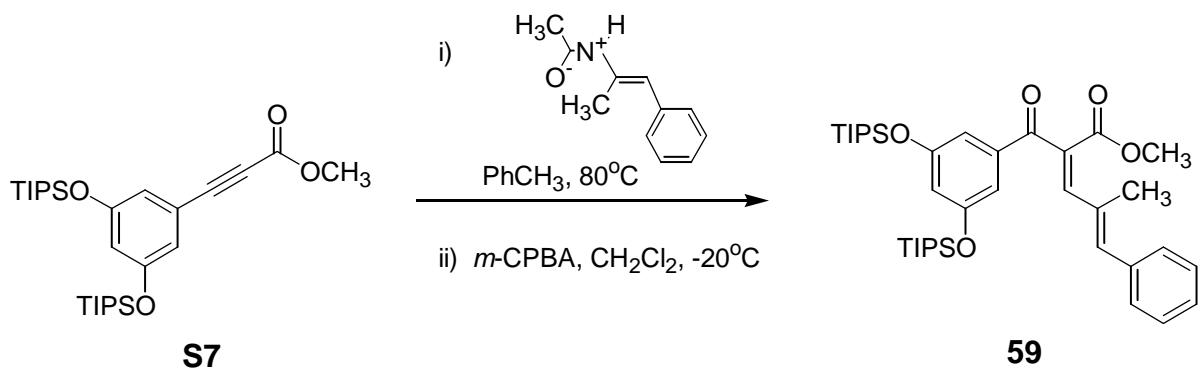

General Procedure III: Nitrone 3+2/Oxidative Extrusion Sequence Leading to Alkylidene $\beta$-Ketoester 59: In a dry $100 \mathrm{~mL}$ round-bottom flask was weighed the nitrone (1.40 g, $8.0 \mathrm{mmol})$, S7 (3.58 g, $7.1 \mathrm{mmol})$, and toluene $(35 \mathrm{~mL})$, and the solution was heated at $80^{\circ} \mathrm{C}$ for $16 \mathrm{~h}$. The solution was concentrated, and $\mathrm{CH}_{2} \mathrm{Cl}_{2}(35 \mathrm{~mL})$ was added, cooled to $-20^{\circ} \mathrm{C}$, and $m$-CPBA $(2.51 \mathrm{~g}, 11.6 \mathrm{mmol})$ was added and stirred for 30 min. The reaction was quenched with saturated $\mathrm{Na}_{2} \mathrm{SO}_{3}(50 \mathrm{~mL})$, the layers were separated and the aqueous phase was extracted with $\mathrm{CH}_{2} \mathrm{Cl}_{2}(2 \times 50 \mathrm{~mL})$, the organic phases were combined and washed with brine $(50 \mathrm{~mL})$. The organic phase was dried over $\mathrm{Na}_{2} \mathrm{SO}_{4}$, filtered, and concentrated. The crude material was purified by column chromatography (8:1 hexanes:ethyl acetate), yielding $3.0 \mathrm{~g} \mathrm{(65 \% )} \mathrm{of} 59$.

$\left.{ }^{1} \mathbf{H ~ N M R ~ ( C D C l}_{3}, \mathbf{4 0 0} \mathbf{M H z}\right): \delta 1.12(\mathrm{~d}, \mathrm{~J}=9.0 \mathrm{~Hz}, 36 \mathrm{H}), 1.24(\mathrm{~m}, 6 \mathrm{H}), 1.79(\mathrm{~s}, 3 \mathrm{H})$, $3.69(\mathrm{~s}, 3 \mathrm{H}), 6.65(\mathrm{t}, \mathrm{J}=2.0 \mathrm{~Hz}, 1 \mathrm{H}), 6.98(\mathrm{~s}, 1 \mathrm{H}), 7.07(\mathrm{~d}, \mathrm{~J}=2.4 \mathrm{~Hz}, 2 \mathrm{H}), 7.34(\mathrm{~m}, 5 \mathrm{H})$, $7.66(\mathrm{~d}, \mathrm{~J}=0.8 \mathrm{~Hz}, 1 \mathrm{H}) ;{ }^{13} \mathbf{C} \mathbf{N M R}\left(\mathbf{C D C l}_{3}, \mathbf{1 0 0} \mathbf{M H z}\right): 12.5,17.8,52.3,113.7,117.3$, 128.0, 128.3, 129.2, 129.7, 133.3, 136.2, 138.9, 141.9, 147.8, 157.3, 165.8, 195.0; IR (neat, $\mathbf{~ c m}^{-1}$ ): $3023.7,2944.7,2892.3,1724.2,1713.6,1678.6,1672.0,1587.8,1442.8$, 
1333.8, 1249.2, 1197.6; HRMS (EI) calculated for $\mathrm{C}_{38} \mathrm{H}_{58} \mathrm{O}_{5} \mathrm{Si}_{2} 650.3817$, found 650.3817 .

$\mathrm{Z}^{3} \mathrm{~J}_{\mathrm{C}-\mathrm{H}}=4.2 \mathrm{~Hz}$ for $\mathrm{C}_{\text {ketone }}-\mathrm{H},{ }^{3} \mathrm{~J}_{\mathrm{C}-\mathrm{H}}=7.5 \mathrm{~Hz}$ for $\mathrm{C}_{\text {ester }}-\mathrm{H}$.

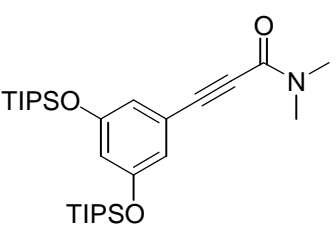

S8

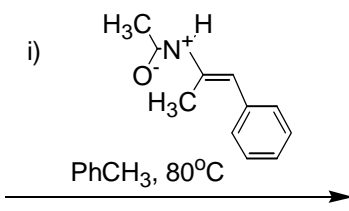

ii) $m$-CPBA, $\mathrm{CH}_{2} \mathrm{Cl}_{2},-20^{\circ} \mathrm{C}$

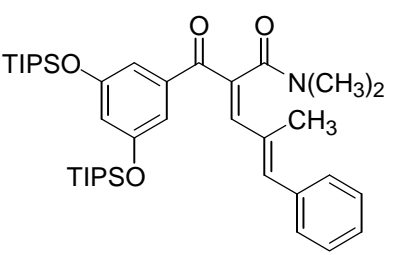

61

Alkylidene $\beta$-Ketoamide 61: Alkynyl amide $\mathbf{S 8}(1.55 \mathrm{~g}, 2.8 \mathrm{mmol})$ was subjected to General Procedure III to obtain $1.21 \mathrm{~g}(63 \%)$ of $\mathbf{6 1}$.

${ }^{1} \mathbf{H}$ NMR $\left(\mathbf{C D C l}_{3}, \mathbf{4 0 0} \mathbf{M H z}\right): \delta 1.10(\mathrm{~d}, \mathrm{~J}=9.0 \mathrm{~Hz}, 36 \mathrm{H}), 1.24(\mathrm{~m}, 6 \mathrm{H}), 2.08(\mathrm{~s}, 3 \mathrm{H})$, $3.01(\mathrm{~s}, 3 \mathrm{H}), 3.08(\mathrm{~s}, 3 \mathrm{H}), 6.62(\mathrm{t}, \mathrm{J}=2.0 \mathrm{~Hz}, 1 \mathrm{H}), 6.79(\mathrm{~s}, 1 \mathrm{H}), 6.88(\mathrm{~d}, \mathrm{~J}=2.4 \mathrm{~Hz}, 2 \mathrm{H})$, $7.02(\mathrm{~s}, 1 \mathrm{H}), 7.34(\mathrm{~m}, 5 \mathrm{H}) ;{ }^{13} \mathbf{C} \mathbf{~ N M R}\left(\mathbf{C D C l}_{3}, \mathbf{1 0 0} \mathbf{~ M H z}\right): 12.6,14.6,17.8,34.6,38.2$, 113.9, 115.5, 128.1, 128.3, 129.5, 133.7, 134.4, 136.3, 139.4, 141.5, 147.9, 156.9, 168.0, 194.2; IR (neat, $\mathbf{~ c m}^{-1}$ ): 2944.9, 2867.1, 1643.3, 1584.6, 1436.5, 1392.7, 1336.0, 1238.7, 1071.9; HRMS (EI) calculated for $\mathrm{C}_{39} \mathrm{H}_{61} \mathrm{NO}_{4} \mathrm{Si}_{2}(\mathrm{M}+\mathrm{Na})^{+}:$: 686.4031, found: 686.4056. $\mathrm{Z}^{3} \mathrm{~J}_{\mathrm{C}-\mathrm{H}}=2.5 \mathrm{~Hz}$ for $\mathrm{C}_{\text {ketone }}-\mathrm{H},{ }^{3} \mathrm{~J}_{\mathrm{C}-\mathrm{H}}=10.5 \mathrm{~Hz}$ for $\mathrm{C}_{\text {ester }}-\mathrm{H}$.
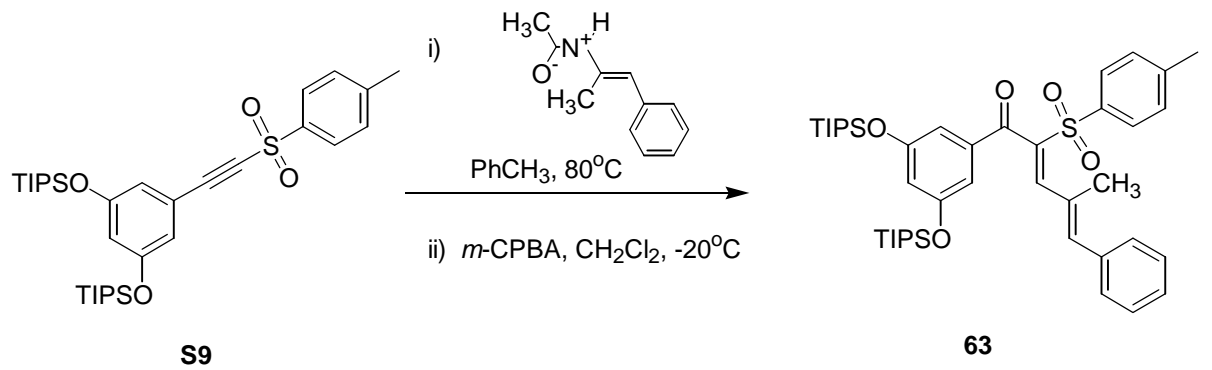

Alkylidene $\boldsymbol{\alpha}$-Ketosulfone 63: Alkynyl sulfone S9 (739 mg, $1.2 \mathrm{mmol}$ ) was subjected to General Procedure III to obtain $191 \mathrm{mg}(43 \%)$ of 63.

${ }^{1}{ }_{\text {H NMR }}\left(\mathbf{C D C l}_{3}, \mathbf{4 0 0} \mathbf{M H z}\right): \delta 1.10(\mathrm{~d}, \mathrm{~J}=9.0 \mathrm{~Hz}, 36 \mathrm{H}), 1.24(\mathrm{~m}, 6 \mathrm{H}), 1.68(\mathrm{~s}, \mathrm{~J}=1.0$ $\mathrm{Hz}, 3 \mathrm{H}), 2.42(\mathrm{~s}, 3 \mathrm{H}), 6.64(\mathrm{t}, \mathrm{J}=2.0 \mathrm{~Hz}, 1 \mathrm{H}), 6.92(\mathrm{~s}, 1 \mathrm{H}), 7.03(\mathrm{~d}, \mathrm{~J}=2.0 \mathrm{~Hz}, 2 \mathrm{H}), 7.17$ $(\mathrm{d}, \mathrm{J}=8.0 \mathrm{~Hz}, 2 \mathrm{H}), 7.30(\mathrm{~m}, 5 \mathrm{H}), 7.69(\mathrm{~d}, \mathrm{~J}=0.5 \mathrm{~Hz}, 1 \mathrm{H}), 7.74(\mathrm{~d}, \mathrm{~J}=8.0 \mathrm{~Hz}, 2 \mathrm{H}) ;{ }^{13} \mathrm{C}$ NMR (CDCl ${ }_{3}, \mathbf{1 0 0}$ MHz): 12.5, 16.2, 17.4, 21.6, 114.2, 117.8, 128.1, 128.3, 129.0, 129.6, 130.2, 131.9, 135.7, 137.3, 138.2, 139.0, 142.5, 144.4, 145.8, 157.3, 176.5, 191.6; IR (neat, $\mathbf{~ c m}^{\mathbf{- 1}}$ ): 2948.1, 2728.9, 1745.9, 1666.1, 1453.3, 1243.8, 1086.5; HRMS (EI) calculated for $\mathrm{C}_{43} \mathrm{H}_{62} \mathrm{O}_{5} \mathrm{SSi}_{2}(\mathrm{M}+\mathrm{Na})^{+}$: 769.3749 , found 769.3740 .

$\mathrm{Z}^{3} \mathrm{~J}_{\mathrm{C}-\mathrm{H}}=8.8 \mathrm{~Hz}$ for $\mathrm{C}_{\mathrm{ketone}}-\mathrm{H}$. 


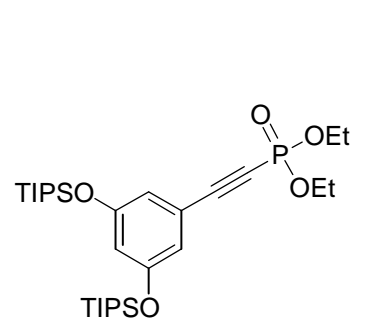

S10

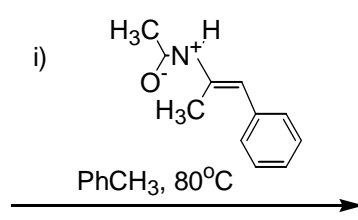

ii) $m$-CPBA, $\mathrm{CH}_{2} \mathrm{Cl}_{2},-20^{\circ} \mathrm{C}$

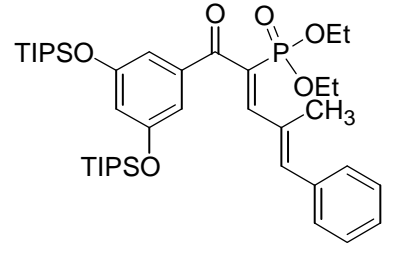

65

Alkylidene $\alpha$-Ketophosphate 65: Alkynyl phosphate S10 (2.70 g, $4.5 \mathrm{mmol})$ was subjected to General Procedure III to obtain $1.85 \mathrm{~g}(57 \%)$ of 65.

${ }^{1} \mathbf{H}$ NMR (CDCl ${ }_{3}, 400$ MHz): $\delta 1.12(\mathrm{~d}, \mathrm{~J}=9.0 \mathrm{~Hz}, 36 \mathrm{H}), 1.24(\mathrm{~m}, 12 \mathrm{H}), 1.75(\mathrm{~s}, 3 \mathrm{H})$, 4.13 (quint., $\mathrm{J}=7.2 \mathrm{~Hz}, 4 \mathrm{H}), 6.64(\mathrm{t}, \mathrm{J}=2.0 \mathrm{~Hz}, 1 \mathrm{H}), 6.87(\mathrm{~s}, 1 \mathrm{H}), 7.11(\mathrm{~d}, \mathrm{~J}=2.0 \mathrm{~Hz}$, 2H), $7.27(\mathrm{~m}, 5 \mathrm{H}), 7.47(\mathrm{~d}, \mathrm{~J}=26.0 \mathrm{~Hz}, 1 \mathrm{H}) ;{ }^{13} \mathbf{C}$ NMR (CDCl $\left.\mathbf{3}, \mathbf{1 0 0} \mathbf{M H z}\right): 12.5,16.1$, 17.8, 62.6, 114.0, 114.3, 117.3, 127.9, 128.2, 129.3, 133.6, 133.8, 136.2, 139.0, 140.4, 151.2, 155.9, 157.2, 195.0; IR (neat, $\mathbf{~ c m}^{-1}$ ): 2868.0, 2728.0, 2359.3, 1665.8, 1587.1, 1444.5, 1334.0, 1172.9, 1026.7; HRMS (EI) calculated for $\mathrm{C}_{40} \mathrm{H}_{65} \mathrm{O}_{6} \mathrm{PSi}_{2}(\mathrm{M}+\mathrm{Na})^{+}$: 751.3950 , found: 751.3964 .

$Z^{3} \mathrm{~J}_{\mathrm{C}-\mathrm{H}}=7.8 \mathrm{~Hz}$ for $\mathrm{C}_{\text {ketone }}-\mathrm{H}$.

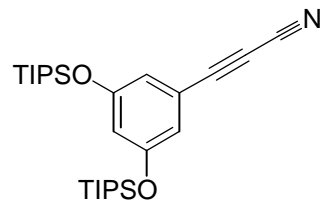

S11

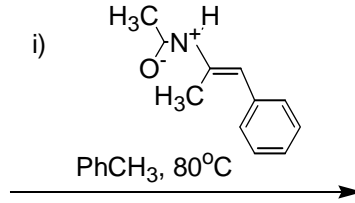

ii) $m-\mathrm{CPBA}, \mathrm{CH}_{2} \mathrm{Cl}_{2},-20^{\circ} \mathrm{C}$

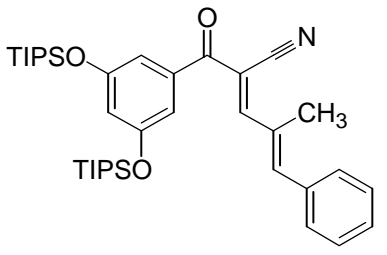

67

Alkylidene $\alpha$-Ketonitrile 67: Alkynyl nitrile S11 (2.41 g, $5.1 \mathrm{mmol})$ was subjected to General Procedure III to obtain $1.80 \mathrm{~g}(57 \%)$ of 67.

${ }^{1} \mathbf{H}$ NMR (CDCl 3 , 400 MHz): $\delta 1.10(\mathrm{~d}, \mathrm{~J}=9.0 \mathrm{~Hz}, 36 \mathrm{H}), 1.24(\mathrm{~m}, 6 \mathrm{H}), 2.50(\mathrm{~s}, 3 \mathrm{H})$, $6.65(\mathrm{t}, \mathrm{J}=2.0 \mathrm{~Hz}, 1 \mathrm{H}), 6.90(\mathrm{~d}, \mathrm{~J}=2.0 \mathrm{~Hz}, 2 \mathrm{H}), 7.09(\mathrm{~s}, 1 \mathrm{H}), 7.41(\mathrm{~m}, 5 \mathrm{H}), 7.68(\mathrm{~s}, 1 \mathrm{H})$; ${ }^{13} \mathbf{C}$ NMR (CDCl 3 , 100 MHz): 12.6, 15.6, 17.9, 109.5, 113.7, $116.5\left(\mathrm{~d},{ }^{3} \mathrm{~J}_{\mathrm{CH}}=13.4 \mathrm{~Hz}\right)$, 128.6, 129.3, 130.0, 133.8, 135.4, 137.7, 147.5, 157.2, 160.3, 189.6; IR (neat, $\mathbf{~ c m}^{\mathbf{- 1}}$ ): 2943.2, 2866.0, 2210.3 1554.5, 1580.6, 1434.9, 1171.7, 1029.9; HRMS (EI) calculated for $\mathrm{C}_{37} \mathrm{H}_{55} \mathrm{NO}_{3} \mathrm{Si}_{2} 617.3715$, found 617.3743.

$Z^{3} \mathrm{~J}_{\mathrm{C}-\mathrm{H}}=6.0 \mathrm{~Hz}$ for $\mathrm{C}_{\mathrm{ketone}}-\mathrm{H},{ }^{3} \mathrm{~J}_{\mathrm{C}-\mathrm{H}}=13.4 \mathrm{~Hz}$ for $\mathrm{C}_{\mathrm{ester}}-\mathrm{H}$.

The following compounds were prepared according to the following General Procedure for Nazarov cyclization:<smiles>COC(=O)/C(=C\C(C)=C\c1ccccc1)C(=O)c1cc(OS)cc(OS)c1</smiles>

59

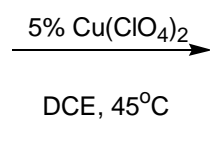

DCE, $45^{\circ} \mathrm{C}$

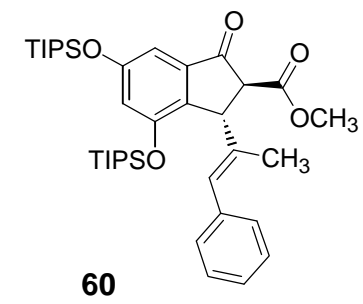




\section{General Procedure IV: Preparation of $\beta$-ketoester via Nazarov cyclization of}

alkylidene $\beta$-ketoester 60: In a dry $10 \mathrm{~mL}$ round-bottom flask was weighed $\mathrm{Cu}\left(\mathrm{ClO}_{4}\right)_{2}$ (7.0 mg, $0.03 \mathrm{mmol})$, and a solution of $59(341 \mathrm{mg}, 0.5 \mathrm{mmol})$ in DCE (5 mL) was added. The solution was stirred at $45^{\circ} \mathrm{C}$ and monitored by NMR (aliquots were periodically removed from the reaction, filtered through Celite with $\mathrm{CH}_{2} \mathrm{Cl}_{2}$, and concentrated) for $8 \mathrm{~h}$, then the reaction mixture was filtered through a plug of silica gel and flushed with $\mathrm{CH}_{2} \mathrm{Cl}_{2}$ concentrated. The crude material was purified by column chromatography (10:1 petroleum ether:ether), yielding $272 \mathrm{mg}(80 \%)$ of $\mathbf{6 0}$.

${ }^{1} \mathbf{H}$ NMR (CDCl 3 , 400 MHz): $\delta 1.12(\mathrm{~d}, \mathrm{~J}=9.0 \mathrm{~Hz}, 36 \mathrm{H}), 1.24(\mathrm{~m}, 6 \mathrm{H}), 1.64(\mathrm{~s}, 3 \mathrm{H})$, $3.49(\mathrm{~d}, \mathrm{~J}=3.2 \mathrm{~Hz}, 1 \mathrm{H}), 3.78(\mathrm{~s}, 3 \mathrm{H}), 4.46(\mathrm{~d}, \mathrm{~J}=3.2 \mathrm{~Hz}, 1 \mathrm{H}), 6.26(\mathrm{~s}, 1 \mathrm{H}), 6.66(\mathrm{~d}, \mathrm{~J}=$ $2.0 \mathrm{~Hz}, 1 \mathrm{H}), 6.88(\mathrm{~d}, \mathrm{~J}=2.0 \mathrm{~Hz}, 1 \mathrm{H}), 7.24(\mathrm{~m}, 5 \mathrm{H}) ;{ }^{13} \mathbf{C} \mathbf{N M R}\left(\mathbf{C D C l}_{3}, \mathbf{1 0 0} \mathbf{M H z}\right): 12.5$, $17.8,50.0,52.8,61.0,106.7,117.3,126.2,127.9,128.8,137.4,137.6,138.2,138.3$, 154.5, 157.7, 169.2, 198.7; IR (neat, $\mathbf{~ c m}^{-1}$ ): 2945.1, 2891.1, 2866.0, 1747.4, 1712.7, 1606.6, 1479.3, 1348.2, 1180.4; HRMS (EI) calculated for $\mathrm{C}_{38} \mathrm{H}_{58} \mathrm{O}_{5} \mathrm{Si}_{2} 650.3817$, found 650.3817 .

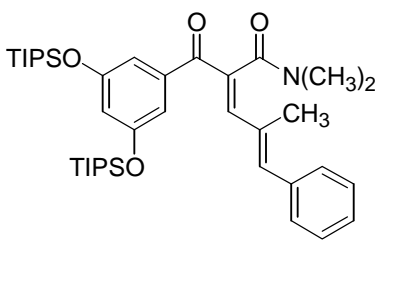

61

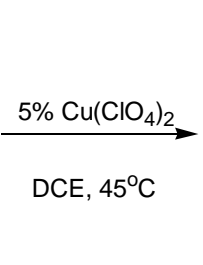

DCE, $45^{\circ} \mathrm{C}$

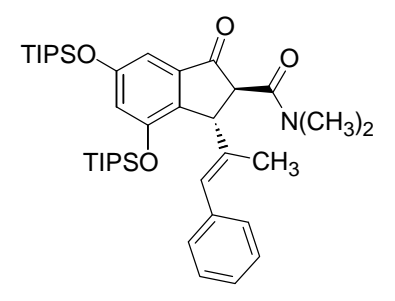

62

$\beta$-Ketoamide 62: Alkylidene $\beta$-ketoamide 61 (100 $\mathrm{mg}, 0.15 \mathrm{mmol}$ ) was subjected to General Procedure IV at $45^{\circ} \mathrm{C}$ for $14 \mathrm{~h}$ to yield $92 \mathrm{mg}(92 \%)$ of 62.

${ }^{1} \mathbf{H}$ NMR (CDCl 3 , 400 MHz): $\delta 1.12(\mathrm{~d}, \mathrm{~J}=9.0 \mathrm{~Hz}, 36 \mathrm{H}), 1.24(\mathrm{~m}, 6 \mathrm{H}), 1.76(\mathrm{~d}, \mathrm{~J}=1.2$ $\mathrm{Hz}, 3 \mathrm{H}), 3.05(\mathrm{~s}, 3 \mathrm{H}), 3.28(\mathrm{~s}, 3 \mathrm{H}), 3.82(\mathrm{~d}, \mathrm{~J}=3.2 \mathrm{~Hz}, 1 \mathrm{H}), 4.70(\mathrm{~d}, \mathrm{~J}=3.2 \mathrm{~Hz}, 1 \mathrm{H})$, $6.32(\mathrm{~s}, 1 \mathrm{H}), 6.63(\mathrm{~d}, \mathrm{~J}=2.0 \mathrm{~Hz}, 1 \mathrm{H}), 6.82(\mathrm{~d}, \mathrm{~J}=2.0 \mathrm{~Hz}, 1 \mathrm{H}), 7.35(\mathrm{~m}, 5 \mathrm{H}) ;{ }^{13} \mathbf{C} \mathbf{N M R}$ $\left(\mathbf{C D C l}_{3}, \mathbf{1 0 0} \mathbf{~ M H z}\right): 12.6,13.0,16.0,17.8,36.2,38.2,50.3,58.7,106.3,114.6,117.2$, $126.2,126.5,128.0,128.8,137.8,138.1,138.6,139.1,154.5,157.0,157.5,167.9,201.1$; IR (neat, $\mathbf{~ c m}^{-1}$ ): $2943.2,2866.0,1716.5,1647.1,1338.5,1170.7$; HRMS (EI) calculated for $\mathrm{C}_{39} \mathrm{H}_{61} \mathrm{NO}_{4} \mathrm{Si}_{2}(\mathrm{M}+\mathrm{Na})^{+}: 686.4031$, found 686.4022 .

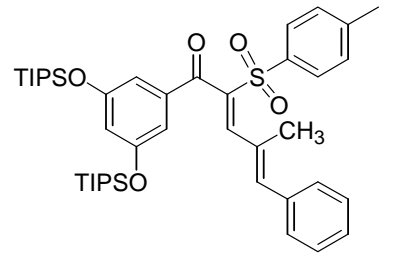

63

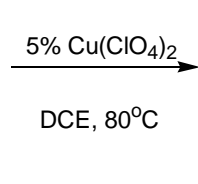

DCE, $80^{\circ} \mathrm{C}$

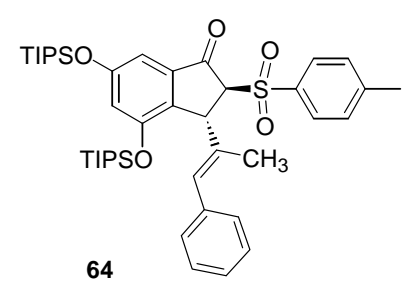

$\alpha$-Ketosulfone 64: Alkylidene $\alpha$-ketosulfone 63 (163 $\mathrm{mg}, 0.22 \mathrm{mmol})$ was subjected to General Procedure IV at $80^{\circ} \mathrm{C}$ for $7 \mathrm{~h}$ to yield $139 \mathrm{mg}(85 \%)$ of 64 .

${ }^{1} \mathrm{H}$ NMR (CDCl 3 , $\left.400 \mathbf{M H z}\right): \delta 1.12(\mathrm{~d}, \mathrm{~J}=9.0 \mathrm{~Hz}, 36 \mathrm{H}), 1.24(\mathrm{~m}, 6 \mathrm{H}), 1.79(\mathrm{~d}, \mathrm{~J}=0.8$ $\mathrm{Hz}, 3 \mathrm{H}), 2.39$ (s, 3H), $3.94(\mathrm{~d}, \mathrm{~J}=2.0 \mathrm{~Hz}, 1 \mathrm{H}), 4.58(\mathrm{~d}, \mathrm{~J}=1.2 \mathrm{~Hz}, 1 \mathrm{H}), 6.03(\mathrm{~s}, 1 \mathrm{H})$, $6.58(\mathrm{~d}, \mathrm{~J}=2.0 \mathrm{~Hz}, 1 \mathrm{H}), 6.79(\mathrm{~d}, \mathrm{~J}=2.0 \mathrm{~Hz}, 1 \mathrm{H}), 7.09$ (d, J = 7.2 Hz, 2H), $7.27(\mathrm{~m}, 5 \mathrm{H})$, $7.73(\mathrm{~d}, \mathrm{~J}=8.0 \mathrm{~Hz}, 2 \mathrm{H}) ;{ }^{13} \mathbf{C} \mathbf{N M R}\left(\mathbf{C D C l}_{3}, \mathbf{1 0 0} \mathbf{M H z}\right): 12.5,16.5,17.8,21.7,47.5$, 76.1, 106.5, 117.4, 126.4, 127.5, 127.9, 128.8, 129.2, 129.6, 134.4, 136.6, 137.3, 138.7, 
145.1, 154.1, 158.0, 194.3; IR (neat, $\mathbf{c m}^{-1}$ ): 2943.7, 2865.5, 1718.9, 1604.2, 1477.9, 1325.5, 1175.5, 1148.5; HRMS (EI) calculated for $\mathrm{C}_{43} \mathrm{H}_{62} \mathrm{O}_{5} \mathrm{SSi}_{2}(\mathrm{M}+\mathrm{Na})^{+}: 769.3749$, found: 769.3777 .<smiles>CCOP(=O)(CC)C(=CC(=Cc1ccccc1)C(=O)c1cc(OC)cc(OC(F)(F)F)c1)C(=O)OC</smiles>

65

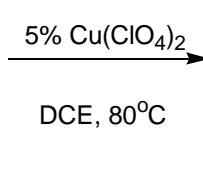

DCE, $80^{\circ} \mathrm{C}$

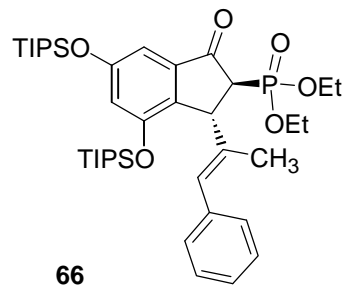

$\alpha$-Ketophosphate 66: Alkylidene $\alpha$-ketophosphate 65 (88.3 mg, $0.12 \mathrm{mmol})$ was subjected to General Procedure IV at $80^{\circ} \mathrm{C}$ for $24 \mathrm{~h}$ to yield $78 \mathrm{mg}(90 \%)$ of 66.

${ }^{1} \mathbf{H}$ NMR (CDCl 3 , 400 MHz): $\delta 1.12(\mathrm{~d}, \mathrm{~J}=9.0 \mathrm{~Hz}, 36 \mathrm{H}), 1.24(\mathrm{~m}, 12 \mathrm{H}), 1.72(\mathrm{~s}, 3 \mathrm{H})$, $3.06(\mathrm{dd}, \mathrm{J}=1.6 \mathrm{~Hz}, 26.4 \mathrm{~Hz}, 1 \mathrm{H}), 4.14(\mathrm{~m}, 4 \mathrm{H}), 4.48(\mathrm{~d}, \mathrm{~J}=16.4 \mathrm{~Hz}, 1 \mathrm{H}), 6.26(\mathrm{~s}, 1 \mathrm{H})$, $6.64(\mathrm{~d}, \mathrm{~J}=1.6 \mathrm{~Hz}, 1 \mathrm{H}), 6.89(\mathrm{~d}, \mathrm{~J}=1.6 \mathrm{~Hz}, 1 \mathrm{H}), 7.16(\mathrm{~m}, 2 \mathrm{H}), 7.27(\mathrm{~m}, 3 \mathrm{H}) ;{ }^{13} \mathbf{C} \mathbf{N M R}$ $\left(\mathbf{C D C l}_{3}, \mathbf{1 0 0} \mathbf{M H z}\right):$ 12.6, 15.7, 16.3, 17.8, 48.0, 53.9, 55.2, 62.7, 63.0, 106.5, 116.9, 126.2, 126.8, 128.0, 128.8, 137.7, 138.2, 139.4, 154.3, 157.7, 198.8; IR (neat, $\mathbf{~ c m}^{-1}$ ): 2943.7, 2931.1, 2866.0, 1715.6, 1604.7, 1476.4, 1470.6, 1345.7, 1175.1, 1051.6; HRMS (EI) calculated for $\mathrm{C}_{40} \mathrm{H}_{65} \mathrm{O}_{6} \mathrm{PSi}_{2}(\mathrm{M}+\mathrm{Na})^{+}:$751.3950, found: 751.3967 .
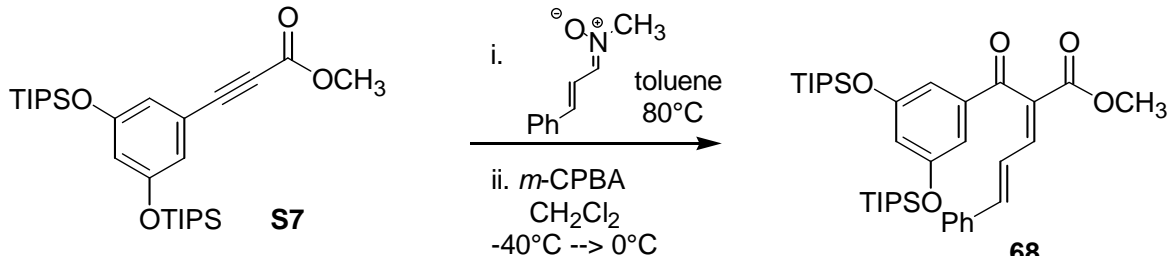

Alkylidene $\beta$-Ketoester 68: Alkynyl ester S7 (0.75 g, $1.5 \mathrm{mmol})$ was reacted under the conditions of General Procedure III using the nitrone of cinnamaldehyde to yield $320 \mathrm{mg}$ $(67 \%)$ of 68.

${ }^{1}$ H NMR (500 MHz, $\left.\mathbf{C D C l}_{3}\right): \delta 7.69(\mathrm{~d}, \mathrm{~J}=12 \mathrm{~Hz}, 1 \mathrm{H}), 7.34(\mathrm{~m}, 5 \mathrm{H}), 7.05(\mathrm{~d}, \mathrm{~J}=2.5$ $\mathrm{Hz}, 2 \mathrm{H}), 7.02(\mathrm{~d}, \mathrm{~J}=16 \mathrm{~Hz}, 1 \mathrm{H}), 6.70(\mathrm{dd}, \mathrm{J}=2.5,3.0 \mathrm{~Hz}, 1 \mathrm{H}), 6.66(\mathrm{t}, \mathrm{J}=2.4 \mathrm{~Hz}, 1$ $\mathrm{H}), 3.71(\mathrm{~s}, 3 \mathrm{H}), 1.26(\mathrm{~m}, 6 \mathrm{H}), 1.10(\mathrm{~d}, \mathrm{~J}=8.5 \mathrm{~Hz}, 18 \mathrm{H}) ;{ }^{13} \mathbf{C}$ NMR (100 MHz, $\left.\mathbf{C D C l}_{3}\right):$ 194.0, 176.6, 165.4, 157.4, 143.6, 143.4, 138.7, 135.5, 131.0, 129.6, 128.7, 127.6, 123.0, 117.5, 113.9, 52.2, 17.8, 12.6; IR (neat, $\mathbf{~ c m}^{-1}$ ): 2944.1, 2891.1, 2866.0, $1719.4,1672.2$, 1618.2, 1583.5, 1461.9, 1437.8, 1365.5, 1334.7, 1303.8, 1278.7, 1231.5, 1213.1, 1170.7, 1150.5, 1077.2, 1028.0, 1014.5, 998.1, 988.5, 974.0, 953.7, 919.0;

HRMS (EI) calculated for $\mathrm{C}_{37} \mathrm{H}_{56} \mathrm{O}_{5} \mathrm{Si}_{2}(\mathrm{M}+\mathrm{Na})^{+}: \quad 659.3558$, found: 659.3542 .

$\boldsymbol{E}^{3} \mathrm{~J}_{\mathrm{C}-\mathrm{H}}=9.0 \mathrm{~Hz}$ for $\mathrm{C}_{\text {ketone }}-\mathrm{H},{ }^{3} \mathrm{~J}_{\mathrm{C}-\mathrm{H}}=6.6 \mathrm{~Hz}$ for $\mathrm{C}_{\text {ester }}-\mathrm{H}$.<smiles>COC(=O)/C(=C/C=C\c1cc(O[18F])cc(O[18F])c1)C(=O)c1ccccc1</smiles>

68
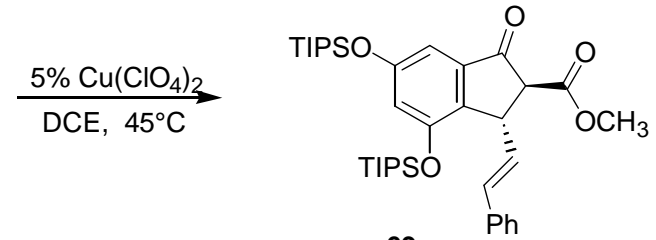

69

$\beta$-Ketoester 69: Alkylidene $\beta$-ketoester 68 ( $0.15 \mathrm{~g}, 0.24 \mathrm{mmol})$ was subjected to General Procedure IV for $26 \mathrm{~h}$ at $45^{\circ} \mathrm{C}$ to yield $0.13 \mathrm{~g}(83 \%)$ of 69 . 
${ }^{1}$ H NMR (400 MHz, $\left.\mathbf{C D C l}_{3}\right): \delta 7.27(\mathrm{~m}, 3 \mathrm{H}), 7.23(\mathrm{~m}, 2 \mathrm{H}), 6.86(\mathrm{~d}, \mathrm{~J}=2.0 \mathrm{~Hz}, 1 \mathrm{H})$, $6.65(\mathrm{~d}, \mathrm{~J}=2.0 \mathrm{~Hz}, 1 \mathrm{H}), 6.49(\mathrm{~m}, 1 \mathrm{H}), 6.32(\mathrm{dd}, \mathrm{J}=7.6,16 \mathrm{~Hz}, 1 \mathrm{H}), 4.53(\mathrm{dd}, \mathrm{J}=3.2$, $8.0 \mathrm{~Hz}, 1 \mathrm{H}), 3.71(\mathrm{~s}, 3 \mathrm{H}), 3.57(\mathrm{~d}, \mathrm{~J}=3.2 \mathrm{~Hz}, 1 \mathrm{H}), 1.32(\mathrm{~m}, 6 \mathrm{H}), 1.10(\mathrm{~d}, \mathrm{~J}=8.5 \mathrm{~Hz}$, $18 \mathrm{H}) ;{ }^{13} \mathbf{C}$ NMR (100 MHz, $\left.\mathbf{C D C l}_{3}\right):$ 198.3, 168.9, 157.7, 154.5, 138.2, 137.4, 136.7, 131.2 , 129.4, 128.4, 128.2, 127.4, 126.1, 126.0, 117.5, 106.6, 61.3, 52.7, 43.5, 39.1, 17.8, 12.9; IR (neat, $\mathbf{~ c m}^{-1}$ ): 2944.1, 2891.1, 2866.0, 1744.5, 1716.5, 1654.8, 1650.0, 1603.7, $1577.7,1556.5,1474.5,1462.9,1448.4,1434.0,1389.6,1384.8,1346.2,1288.4,1237.3$, 1205.4, 1175.5, 1155.3, 1091.6, 1068.5, 1028.0, 1013.5, 996.2, 961.5, 955.7, 919.0;

HRMS (EI) calculated for $\mathrm{C}_{37} \mathrm{H}_{56} \mathrm{O}_{5} \mathrm{Si}_{2}(\mathrm{M}+\mathrm{Na})^{+}:$: 659.3558 , found: 659.3549 .

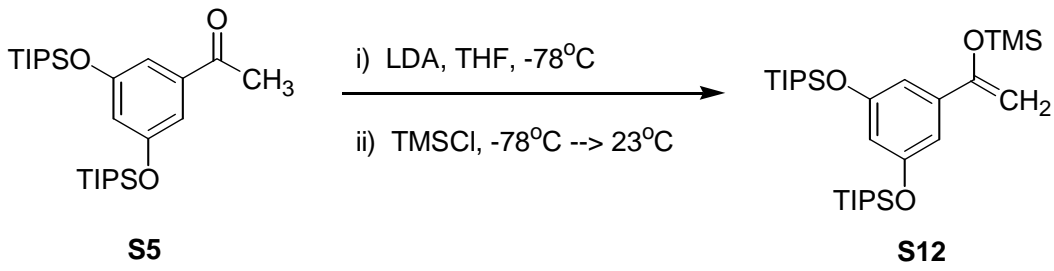

Silyl Enol Ether S12: A dry $25 \mathrm{~mL}$ round-bottom flask was charged with THF (4 mL), $i \mathrm{Pr}_{2} \mathrm{NH}(0.4 \mathrm{~mL}, 2.8 \mathrm{mmol})$, cooled to $0^{\circ} \mathrm{C}$, and $n \mathrm{BuLi}(1.6 \mathrm{M}, 1.6 \mathrm{~mL}, 2.6 \mathrm{mmol})$ was added and the solution was stirred for 30 minutes. The flask was cooled to $-78^{\circ} \mathrm{C}$, and a solution of S5 $(990 \mathrm{mg}, 2.1 \mathrm{mmol})$ in THF $(5 \mathrm{~mL})$ was added and stirred for $6 \mathrm{~h}$. Freshly distilled TMSCl $(0.4 \mathrm{~mL}, 2.8 \mathrm{mmol})$ was added, and the reaction was allowed to warm to $23^{\circ} \mathrm{C}$ over $1.5 \mathrm{~h}$. The reaction was quenched with $\mathrm{NH}_{4} \mathrm{Cl}(20 \mathrm{~mL})$, the layers were separated, and the organic phase was diluted with hexanes $(20 \mathrm{~mL})$ and washed with water $(3 \times 25 \mathrm{~mL})$, then brine $(20 \mathrm{~mL})$. The organic layer was dried over $\mathrm{MgSO}_{4}$, filtered, and concentrated. The crude product was used in the next reaction without further purification.<smiles>C=C(O[Na])c1cc(O[AsH])cc(O[AsH2])c1</smiles>

S12

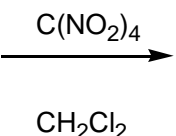

$\mathrm{CH}_{2} \mathrm{Cl}_{2}$

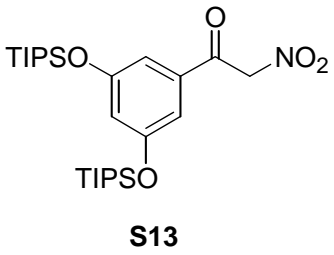

S13

a-Nitroketone S13: ${ }^{7}$ A dry $50 \mathrm{~mL}$ round-bottom flask was charged with $\mathbf{S 1 2}(814 \mathrm{mg}$, $1.5 \mathrm{mmol})$, dry $\mathrm{CH}_{2} \mathrm{Cl}_{2}(6 \mathrm{~mL})$, and wrapped in aluminum foil. Tetranitromethane $(0.2$ $\mathrm{mL}, 1.7 \mathrm{mmol}$ ) was added slowly, and the resulting dark orange solution was stirred for 1 $\mathrm{h}$, at which time the solution had lightened to a bright yellow color. The reaction was quenched with water $(25 \mathrm{~mL})$ and diluted with $\mathrm{CH}_{2} \mathrm{Cl}_{2}(20 \mathrm{~mL})$. The layers were separated and the organic phase was washed repeatedly with water until the aqueous phase was colorless. The organic layer was dried over $\mathrm{Na}_{2} \mathrm{SO}_{4}$, filtered, and concentrated. The crude material was purified by column chromatography (20:1 hexanes: ethyl acetate), yielding $100 \mathrm{mg}(13 \%)$ of $\mathbf{S 1 3}$.

${ }^{1} \mathbf{H}$ NMR (CDCl ${ }_{3}, 400$ MHz): $\delta 1.10(\mathrm{~d}, \mathrm{~J}=9.0 \mathrm{~Hz}, 36 \mathrm{H}), 1.26(\mathrm{~m}, 6 \mathrm{H}), 5.80(\mathrm{~s}, 2 \mathrm{H})$, $6.69(\mathrm{t}, \mathrm{J}=2.0 \mathrm{~Hz}, 1 \mathrm{H}), 6.95(\mathrm{~d}, \mathrm{~J}=2.0 \mathrm{~Hz}, 2 \mathrm{H}) ;{ }^{13} \mathbf{C} \mathbf{N M R}\left(\mathbf{C D C l}_{\mathbf{3}}, \mathbf{1 0 0} \mathbf{~ M H z}\right): 12.6$, 17.8, 81.4, 111.4, 112.5, 118.2, 134.9, 157.8, 185.2; IR (neat, $\mathbf{~ c m}^{-1}$ ): 2945.1, 2922.0, 2866.0, 1705.0, 1583.5, 1566.1, 1436.9, 1342.4, 1172.6. 


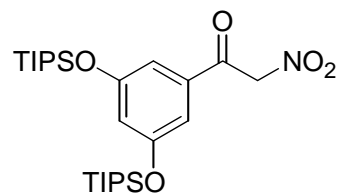

S13
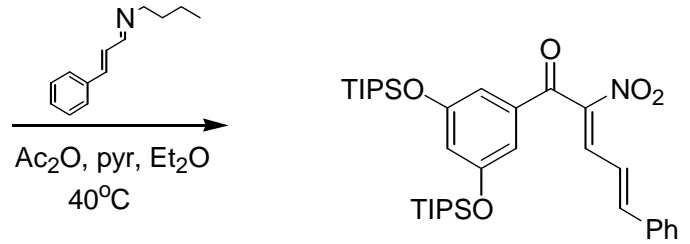

70

Alkylidene $\alpha$-Nitroketone 70: ${ }^{8}$ A pre-formed solution of imine (1.0 M in ether of transcinnamaldehyde, butylamine and $\mathrm{MgSO}_{4}, 0.3 \mathrm{~mL}$ ) was combined with acetic anhydride $(0.05 \mathrm{~mL}, 0.5 \mathrm{mmol})$ and one drop of pyridine in a $10 \mathrm{~mL}$ round-bottom flask, then a solution of S13 (100 mg, $0.2 \mathrm{mmol})$ in ether (1 mL) was added, and the solution was stirred at $40^{\circ} \mathrm{C}$ for $1 \mathrm{~h}$. The reaction was quenched with $\mathrm{NH}_{4} \mathrm{Cl}(10 \mathrm{~mL})$, the layers were separated, and the organic phase was diluted with hexanes $(10 \mathrm{~mL})$ and washed with water $(3 \times 15 \mathrm{~mL})$, then brine $(20 \mathrm{~mL})$. The organic layer was dried over $\mathrm{MgSO}_{4}$, filtered, and concentrated. The crude material was purified by column chromatography (20:1 hexanes:ethyl acetate), yielding $56.2 \mathrm{mg}(45 \%)$ of 70.

${ }^{1} \mathbf{H}$ NMR (CDCl 3 , 400 MHz): $\delta 1.12(\mathrm{~d}, \mathrm{~J}=9.0 \mathrm{~Hz}, 36 \mathrm{H}), 1.24(\mathrm{~m}, 6 \mathrm{H}), 6.68(\mathrm{t}, \mathrm{J}=2.0$ $\mathrm{Hz}, 1 \mathrm{H}), 6.82(\mathrm{dd}, \mathrm{J}=12.0,15.6 \mathrm{~Hz}, 1 \mathrm{H}), 6.98(\mathrm{~d}, \mathrm{~J}=2.0 \mathrm{~Hz}, 2 \mathrm{H}), 7.26(\mathrm{~d}, \mathrm{~J}=15.6 \mathrm{~Hz}$,

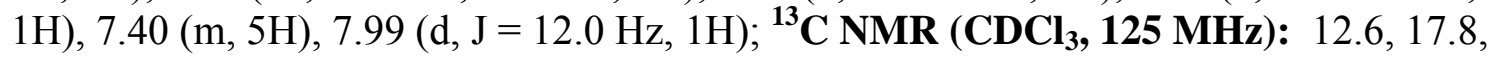
$61.1,113.2$, 113.5, 116.9, 118.1, 119.8, 128.2, 128.4, 129.0, 130.8, 134.9, 137.6, 138.5, 145.7, 148.5, 157.6, 186.8; IR (neat, $\mathbf{~ c m}^{-1}$ ): 2943.7, 2866.0, 1583.0, 1437.4, 1337.1, 1222.8; HRMS (EI) calculated for $\mathrm{C}_{35} \mathrm{H}_{53} \mathrm{NO}_{5} \mathrm{Si}_{2}$ 623.3457, found 623.3445.

$\boldsymbol{E}^{3} \mathrm{~J}_{\mathrm{C}-\mathrm{H}}=6.5 \mathrm{~Hz}$ for $\mathrm{C}_{\text {ketone }}-\mathrm{H}$. 


\section{REFERENCES}

${ }^{1}$ Kingsbury, C.A.; Draney, D.; Sopchik, A.; Rissler, W.; Durham, D. J. Org. Chem. 1976, 41, 3863-3868.

${ }^{2}$ Desimoni, G., Faita, G., Ricci, M., Righetti, P. Tetrahedron 1998, 54, 9581-9602.

${ }^{3}$ Janka, M.; He, W.; Frontier, A.J.; Flaschenriem C.; Eisenberg, R. Tetrahedron 2005, 61, 6193-6206.

${ }^{4}$ Nair, V.; Augustine, A.; Suja, T.D. Synthesis 2002, 15, 2259-2265.

${ }^{5}$ Hughes, T.V.; Cava, M.P. J. Org. Chem. 1999, 64, 313-315.

${ }^{6}$ (a) Canterbury, D.; Frontier, A.J. et al, manuscript submitted, (b) Padwa, A.; Meske, M.; Ni, Z. Tetrahedron, 1995, 51, 89-106, (c) Padwa, A.; Chiacchio, U.; Kline, D.N.; Perumattam, J. J. Org. Chem. 1988, 53, 2238-2245.

${ }^{7}$ Rathore, R.; Lin, Z.; Kochi, J.K. Tetrahedron Lett. 1993, 34, 1859-1862.

8 (a) Boger, D.L.; Lerner, R.A.; Cravatt, B.F. J. Org. Chem. 1994, 59, 5078-5079, (b) Yamamura, K.; Watarai, S.; Kinugasa, T. Bull. Chem. Soc. Japan 1971, 44, 2440-2443, (c) Dornow, A.; Menzel, H. Ann. 1954, 588, 40-44. 